TRAINING THE INTERPRETATION OF AMBIGUITY

BY

\title{
JESSICA CLIFTON
}

\begin{abstract}
A thesis
Submitted to the Victoria University of Wellington in fulfilment of the requirements for the degree of Doctor of Philosophy
\end{abstract}

Victoria University of Wellington

2015 


\section{Declaration}

I hereby declare that this thesis has not been submitted, either in the same or different form, to this or any other university for a degree:

Signature: 


\section{Publications}

Data and literature from this thesis have been reported in the following format and have been published or submitted for publication:

Clifton, J. L., Mountier, E., Tiszai, B., Hedley, S. \& Grimshaw, G. (2015). Practice makes perfect: Training the interpretation of emotional ambiguity. Cognition and Emotion. Advanced online publication. doi: $10.1080 / 02699931.2015 .1020768$

Clifton, J. L., \& Grimshaw, G. (2014, July). Practice makes perfect: Training the interpretation of emotional ambiguity. Poster presented at the International Conference on Cognitive Neuroscience, Brisbane, Australia. 


\begin{abstract}
The interpretation of emotionally ambiguous words, sentences, or scenarios can be biased through training procedures that are collectively called Cognitive Bias Modification for Interpretation (CBM-I). Little CBM-I research has examined the underlying mechanisms responsible for the induction of emotional interpretive biases, or the potential for this line of enquiry to inform psycholinguistic models of meaning activation and selection. In this thesis a novel CBM-I paradigm was developed and then systematically manipulated in order to discriminate between two mechanistic accounts of changes in interpretive bias - the Emotional Priming Account and the Ambiguity Resolution Account. In Experiment 1 participants completed word fragments that were consistently related to either a negative or benign interpretation of an ambiguous sentence. In a subsequent semantic priming task they demonstrated an interpretation bias, in that they were faster to judge the relatedness of targets that were associated with the training-congruent than the training-incongruent meaning of an emotionally ambiguous homograph. In Experiment 2 the time between the presentation of the prime and the target (Stimulus Onset Asynchrony; SOA) at test was shortened. Interpretive biases were not observed at a short SOA suggesting that training did not induce biases at an early lexical activation stage. Interpretive bias was then eliminated when participants simply completed valenced word fragments (Experiment 3) or completed fragments related to emotional but unambiguous sentences (Experiment 4) during training. Only when participants were required to actively resolve emotionally ambiguous sentences during training did changes in interpretation emerge at test. These findings suggest that CBM-I achieves its effects by altering a production rule that aids the selection of a single meaning from alternatives, in line with an Ambiguity Resolution account. Finally, no interpretive biases were observed when the task in the test phase was substituted with a lexical decision task (Experiment 5). Participants only showed biases in the selection of meanings when the test phase encouraged them to interpret the prime. This pattern of results suggests that the alteration of selection patterns in word recognition depend on the strategies employed by participants in the test phase. Overall the findings are discussed with regards to the Ambiguity Resolution and Emotional Priming accounts of modified
\end{abstract}


interpretive biases. Implications for current psycholinguistic models of meaning activation and selection are considered. 


\section{Acknowledgements}

I would like to express my deepest gratitude to my supervisor, Dr. Gina Grimshaw, for her constant guidance and patience, without which this thesis would not have been possible. You have set an amazing example of what it means to be a researcher, a writer and a teacher. Working with you has been a truly inspiring experience and it has been such an honour to learn from you. I would also like to thank my secondary supervisor, Dr Carolyn Wilshire, whose support and insightful feedback has always been gladly welcomed.

This research would not have been possible without the hard work from a number of honours students, Emily, Boglarka and Sophie. Thank you for your long hours of data collection and thoughtful discussions. It was a pleasure to work with all of you.

A big thank you to the great people in the Cognitive and Affective Neuroscience lab: Michael, Laura, Josh, Rosie, Angus, Amy \& Frances. Your support throughout the years has been invaluable. Without you guys this thesis would have been finished years earlier. A very special mention is also required for Hazel Godfrey, a friend quite unlike any other. Your friendship over the years has been so precious to me. You have always known how to brighten my day in the best of ways.

I would like to express my gratitude to my small (but perfectly formed) family. Your love and support during this massive undertaking has meant so much to me. This includes you too, Chris! 


\section{Dedication}

This thesis is dedicated to my father who has been the strongest positive influence in my life. While you may not have fully understood what I have been doing for the past three years you have always provided me with unconditional support and encouragement. Your drive is what inspired me to undertake this task in the first place and your belief in me is what helped me to complete it. Love you Dad. 


\section{List of Tables}

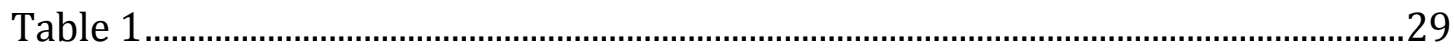

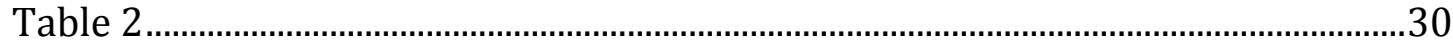

Table 3

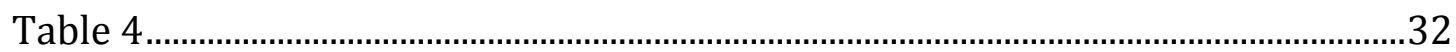

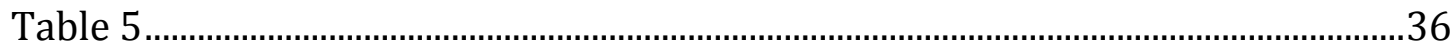

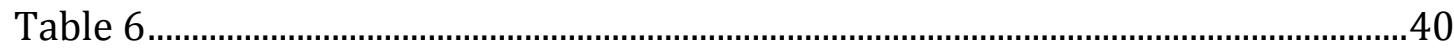

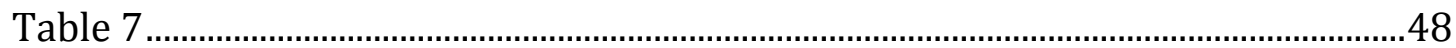

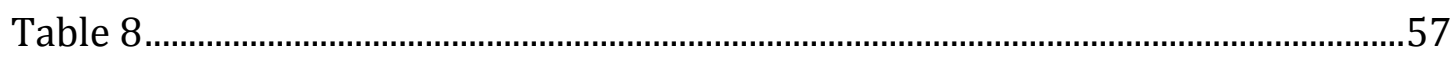

Table 9

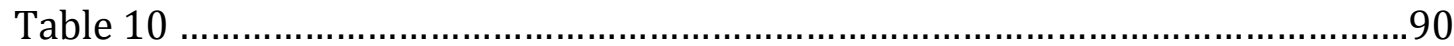

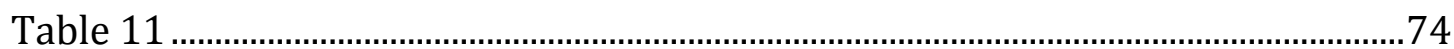




\section{List of Figures}

Figure 1. Typical single word negative training trial (adapted from Grey \& Mathews, 2000)

Figure 2. Typical single word benign training trial (adapted from Grey \& Mathews, 2000)

Figure 3. Lexical Decision trials for words and non-words in a CBM-I test phase (adapted from Grey and Mathews, 2000)

Figure 4. Novel CBM-I Procedure developed for Experiment 1

Figure 5. Response times (ms) to negative and benign targets as a function of training

valence for Experiment 1a. Errors bars are Standard Errors. 38

Figure 6. Response times (ms) to negative and benign targets as a function of training valence for Experiment 1b.

Figure 7. Active and Passive Training Conditions in a CBM-I training phase (adapted from Hoppitt et al., 2010b).

Figure 8. Priming for Negative and Benign Targets (Uncued trial response time Cued trial response time) as a function of Training Valence (Negative or Benign Training). Adapted from Hoppitt et al., (2010b).

Figure 9. Mean response time advantage (and 95\% Confidence Intervals) for Negative Related Targets relative to Benign Related Targets as a function of Training Condition and Experiment. .80 


\section{Contents}

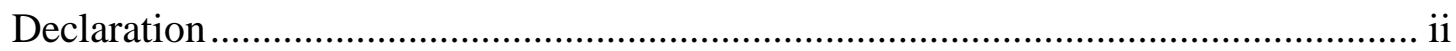

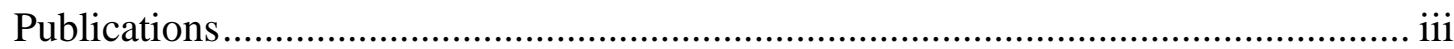

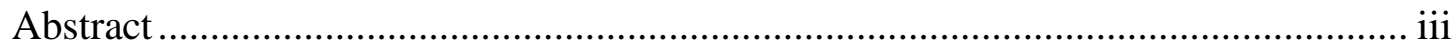

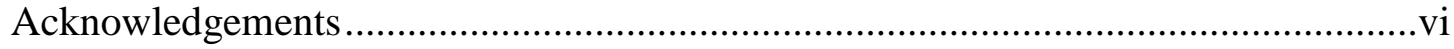

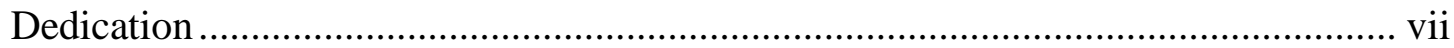

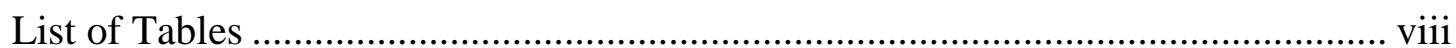

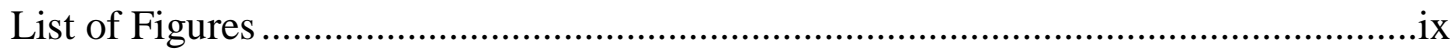

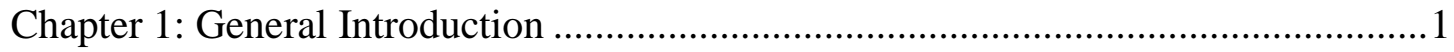

Chapter 2: Developing and Validating an Interpretive Bias Modification Paradigm..26

Chapter 3: Investigating the Time-Course of Interpretive Bias Modification.............44

Chapter 5: Testing Modification of Interpretation with a Lexical Decision.................64

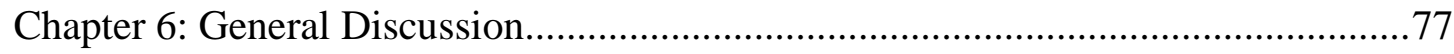

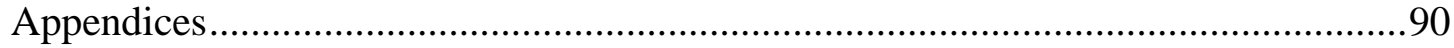




\section{Chapter 1: General Introduction}

Emotional ambiguity is a feature of most, if not all, social interactions. Imagine that a friend expresses their surprise to see you at their party. Do you take this to mean that they are pleased to see you, or do you conclude that they are unhappy with your presence at their event? How you interpret this scenario will depend on many linguistic, individual and situational factors, including your mood at the time, your friend's tone of voice, and the nature of your friendship (Eysenck, Mogg, May, Richards \& Mathews, 1991; Halberstadt, Niedenthal \& Kushner, 1995; Nygaard \& Lunders, 2002). You are also more likely to pick one emotional interpretation over another if you have a stable pre-existing interpretation bias. Typically, healthy people have a slight positivity bias; this means they are more likely (than not) to interpret their friend's words in a positive way. However, some individuals carry a negative bias, so they are more likely to interpret their friend's surprise as disappointment. Negative interpretive biases are common in individuals with anxiety and depression (Butler \& Mathews, 1983; Hirsch \& Mathews, 1997; Lawson, MacLeod \& Hammond, 2002 for a review see Blanchette \& Richards, 2010) and they are considered in many models of mental illness to be instrumental in the onset and maintenance of emotional disorders (e.g., Beck, 1991; Blanchette \& Richards, 2010; Bower, 1981; Clark \& Wells, 1995; Rapee \& Heimberg, 1997). As a result, a novel psychological intervention has been developed that aims to alter negative psychological outcomes by altering interpretive biases.

Cognitive Bias Modification for Interpretation (CBM-I) is a computerised training program that directly manipulates people's interpretive biases and then examines the resulting effects on their mood, symptoms of emotional distress or emotional reactivity (Grey \& Mathews, 2000; Mathews \& Mackintosh, 2000; for reviews see Koster, Fox \& MacLeod, 2009; Woud \& Becker, 2014). A typical CBMI paradigm consists of a training phase to bias the interpretation of emotional ambiguity in a negative or a benign ${ }^{1}$ manner. Following training, changes in

\footnotetext{
1 "Benign" training is used to describe training that contains both positive and neutral stimuli. Typically, training paradigms have negative and benign (instead
} 
participants' mood, emotional reactivity or stress tolerance are assessed in order to determine the causal relationship between interpretive biases and symptoms of mood disorders. Overall, the research suggests that inducing a negative interpretive bias increases symptoms associated with depression and anxiety, and inducing a benign bias alleviates such symptoms (Hallion \& Ruscio, 2011; Mene-Lothmann, et al., 2014). CBM-I is thought to induce these changes in emotional processes by altering the way ambiguous (but potentially emotional) stimuli or events are interpreted. In this thesis, I explore the mechanisms by which training changes interpretation.

\section{Cognitive Bias Modification for Interpretation}

Two studies stand out as seminal in the CBM-I literature, and serve to illustrate two broad classes of paradigms that have been used to manipulate interpretive bias. The first study to administer CBM-I to healthy participants and induce a change in interpretive bias was conducted by Grey and Mathews (2000). In a series of experiments they first trained participants to resolve emotionally ambiguous homographs in either a negative or a benign manner. Participants in the negative training condition repeatedly saw ambiguous primes and solved word fragments related to their negative interpretation e.g.,

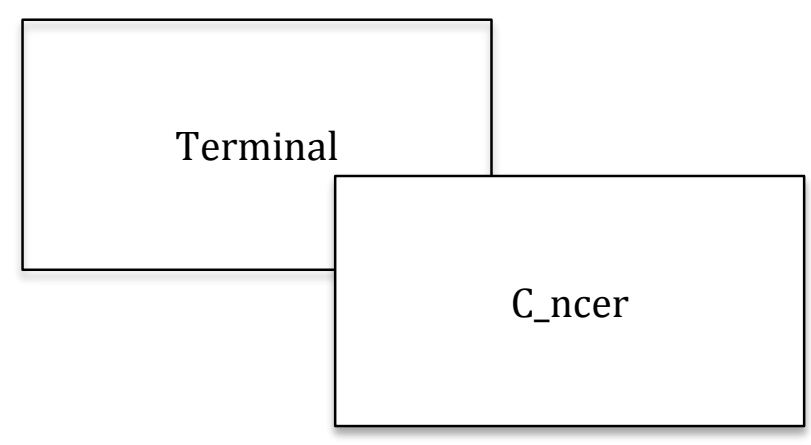

Figure 1. Typical single word negative training trial (adapted from Grey \& Mathews, 2000)

of positive) training conditions, due in part, to the limitations associated with using ambiguous stimuli (i.e., a limited number of ambiguous words have both a negative and a positive, or a negative and a neutral interpretation). Some studies do use a positive training condition and the distinction is referenced explicitly in this thesis. 
Participants in the benign training condition solved word fragments related to the ambiguous prime's benign interpretation e.g.,

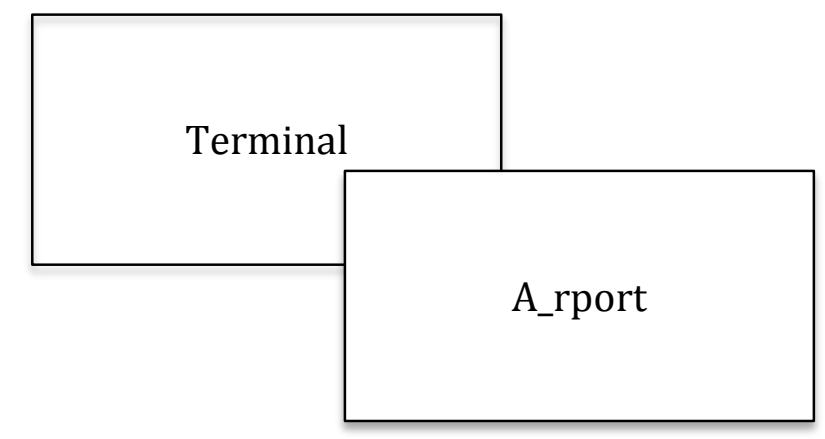

Figure 2. Typical single word benign training trial (adapted from Grey \& Mathews, 2000)

Following training, participants completed a test phase that required them to solve a further set of word fragments, which were related to either the negative or benign interpretation of the ambiguous prime. Participants showed a training valence by target valence interaction that is the hallmark of interpretive bias modification. Participants in the negative training condition were faster to complete negative word fragments relative to benign word fragments, whereas participants in the benign training showed a non-significant advantage to complete fragments that reflected benign interpretations of ambiguous primes. In Experiment 2, participants completed the same fragment completion task in the training phase but completed a primed lexical decision task in the test phase. Participants saw emotionally ambiguous homograph primes, followed $750 \mathrm{~ms}$ later by a target that was either a word or a non-word. Word targets were either negative or benign associates of the ambiguous primes. 
Participants indicated whether the target was a word or a non-word e.g.,

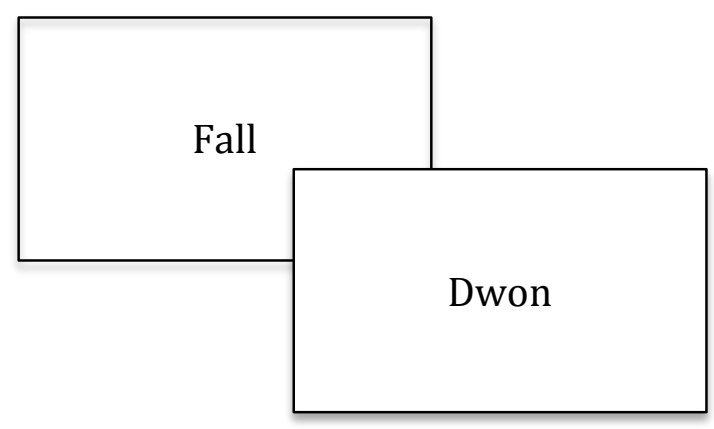

Non-word Trial

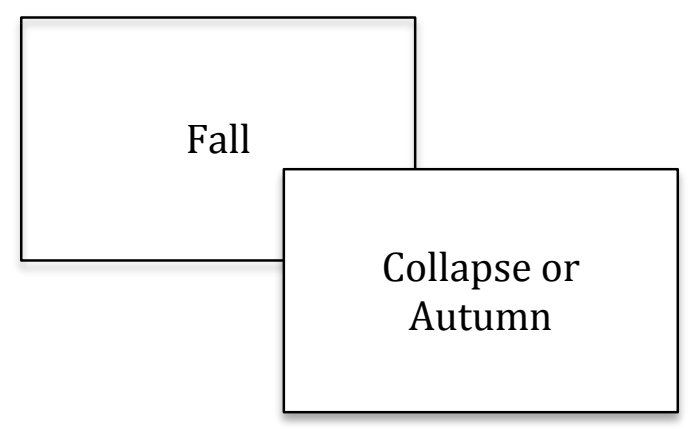

Word Trial

Figure 3. Lexical Decision trials for words and non-words in a CBM-I test phase (adapted from Grey and Mathews, 2000).

The results of the second experiment were similar to those of Experiment 1; participants in the negative training condition were faster to make lexical decisions to training-congruent target words following ambiguous primes, whereas those in the benign training showed no advantage for either valence. The authors concluded that negative interpretive biases normally seen in clinical populations could be mirrored in a healthy sample after a short application of CBM-I training.

A second approach developed by Mathews and Mackintosh (2000) used a scenario-based training method. They used emotional scenarios in the training phase to shift interpretive biases, and a recognition task at test to assess it. During training, participants read ambiguous scenarios that were consistently resolved in either a negative or a positive manner by solving a final word fragment e.g.,

The Dinner Party

Your partner asks you to go to an anniversary dinner that their company is holding. You have not met any of their work colleagues before. Getting ready to go, you think that the new people you will meet will find you:

(B_r_ng/Boring: Negative Training Condition)

(Fr_end_y/Friendly: Positive Training Condition). 
Following several of these scenarios, participants were presented with a booklet containing a series of critical scenarios in which the word fragment did not resolve the ambiguity of the situation. This was considered to be the first stage of the test phase e.g.,

\section{The wedding reception}

Your friend asks you to give a speech at her wedding reception. You prepare some remarks and when the time comes, get to your feet. As you speak, you notice some people in the audience start to l_gh (laugh).

Note that the completion (laugh) does not resolve the scenario as either positive or negative; the resolution depends on the participant's interpretation. After a delay, participants were presented with a second booklet containing four possible interpretations of each critical scenario; these included two target sentences that reflected a possible positive or negative interpretation, and two foil sentences that were also positive or negative but not clearly related to the scenario. Participants were asked to rate each sentence in terms of how similar they were to the original critical scenario. e.g.,

\section{The wedding reception.}

As you speak, some people in the audience start to yawn. (negative foil) As you speak, people in the audience applaud your comments. (positive foil) As you speak, people in the audience find your efforts pathetic. (negative target) As you speak, people in the audience laugh appreciatively. (positive target)

Because the critical scenarios were ambiguous, both negative and benign targets reflected a possible interpretation that could have been drawn from the scenario. Therefore, repeatedly ranking targets of a particular valence as being more similar than the alternative valence would be evidence of an induced interpretive change. Participants in the negative training condition reported higher similarity ratings for negative test sentences over positive test sentences, and vice versa for participants who received positive training (with a strong tendency for all participants to endorse possible interpretations over foils). Participants in the negative training condition also reported higher levels of 
anxiety following training relative to their positively trained counterparts (who showed non-significant alleviations in anxiety symptoms). This suggests that repeatedly reading and completing ambiguous scenarios successfully modified training-congruent interpretive biases, which were linked to negative mood states. Results from both Grey and Matthews (2000) and Mathews and Mackintosh (2000) suggest that interpretive biases (and resulting differences in state anxiety) can be shifted by consistently training participants to disambiguate emotionally ambiguous words or scenarios towards a particular valence.

A large number of CBM-I studies have been conducted in the last decade to replicate and extend the original findings of Grey and Mathews (2000) and Mathews and Mackintosh (2000). The main aim of many CBM-I studies has been to examine the far-transfer effects of training - the ways in which inducing interpretive biases affects clinical outcomes, such as participants' mood or emotional reactivity (Wilson et al., 2006 Beard \& Amir, 2008; Mackintosh, Mathews, Yiend, Ridgeway \& Cook, 2006; Salemink, van den Hout \& Kindt, 2009). Typically far-transfer studies are concerned with how modified interpretive biases translate into changes in responses on emotional tasks that have little methodological overlap with the training task (Hertel \& Mathews, 2011). The main aims of a far-transfer approach in CBM-I research have been to reproduce the emotional biases seen in clinical populations in healthy people, and to test the effectiveness of training in alleviating symptoms associated with depression and anxiety.

The results of far-transfer studies can potentially shed light on the role of interpretive biases in the onset and maintenance of psychopathology, which is characterized by biased cognitive processing. Findings further serve to inform treatment for these disorders by highlighting the protective or adverse effects of emotional interpretive biases on emotional reactivity. For example, studies that have administered CBM-I to anxious populations have found that positive or benign training alleviates self-reported symptoms of anxiety (e.g., Mathews \& Mackintosh, 2000; Hirsch, Mathews \& Clark, 2007; Vassilipoulis, Banerjee \& Prantzalou, 2009). Alternatively, studies that have examined emotional reactivity following negative training have found complementary results. In one study, training showed no effect on anxiety assessed directly after the training phase 
(Mackintosh, Mathews, Yiend, Ridgeway, \& Cook, 2006). However, after viewing videos depicting accidents, participants who had received negative training reported elevations in anxiety and depression while those who had received benign training did not. These findings are in line with the diathesis-stress model of mental health, which posits that cognitive biases are activated by stressful events (e.g., Beck, 1967; Hallion \& Ruscio, 2011; Segal \& Ingram, 1994). In this way CBM-I has been thought to affect an individual's emotional reactivity during the presentation of a stressor but not necessarily affect anxiety or depression perse.

Although there is clear evidence that CBM-I can alter emotional responding, much less research has focused on the near-transfer effects of training, namely the changes in interpretive processes themselves. Near-transfer effects inform us about the underlying mechanisms that support the emergence of an interpretation bias. Typically the benefits of investigating near-transfer effects have been overlooked, and little research has focused on identifying the functional aspects of a CBM-I training paradigm or on understanding exactly how CBM-I engenders a change in interpretive processes. By understanding how CBMI induces interpretive changes in near-transfer tasks, we could develop better training paradigms for the prevention and alleviation of symptoms associated with anxiety and depression. Furthermore, a better understanding of the mechanisms responsible for changes in emotional interpretation should strengthen our current psycholinguistic models of meaning selection and lead to a better understanding of the role of emotion in language comprehension more broadly.

\section{A Review of Common Training and Test Paradigms used in CBM-I Studies}

A number of approaches have been used in CBM-I studies to shift and then probe the presence of interpretive biases. The most common methodological approaches are compared and contrasted below.

\section{Training phases}

Training phases typically require participants to repeatedly disambiguate emotionally ambiguous material in either a consistently negative or benign manner. This can be done at either the scenario level, as used by Mathews and 
Mackintosh (2000), or at the single word level, as used by Grey and Mathews (2000). In scenario-based training, participants typically read paragraphs describing an emotionally ambiguous situation that is resolved only by the completion of a final word fragment (e.g., Mathews \& Mackintosh, 2000). Sometimes the sentence-final word is presented in complete form and participants simply need to read each scenario as it appears on the screen (passive reading tasks e.g., Hoppitt, Mathews, Yiend \& Mackintosh, 2010b). A recent extension of scenario-based training is the inclusion of an imagery component during training, in which participants are instructed to create a mental image of the scenario as it unfolds (e.g., Holmes, Mathews, Dalgleish \& Mackinstosh, 2006; Holmes, Lang \& Shah, 2009; Holmes \& Mathews, 2005; Nelis, Vanbrabant, Holmes \& Raes, 2012). Results from positive imagery-based CBM-I suggests that imagining the scenarios produces greater increases on measures of positive affect (a far-transfer task) versus simply focusing on the verbal descriptions (Holmes, Mathews, Dalgleish \& Mackintosh, 2006). Scenario-based training paradigms have been found to modify interpretive biases using either paragraph or sentence length descriptions of ambiguous events (e.g., Amir \& Taylor, 2012; Beard \& Amir, 2008; Mathews \& Mackintosh, 2000).

In word-level training, participants view homographs (e.g., Terminal) and then are asked to make a response to a target word related to either its negative (e.g., Cancer) or benign (e.g., Airport) interpretation. Tasks include word fragment completion (in which the target is presented in incomplete form and the participant must type in their solution; e.g., Grey and Mathews, 2000), relatedness decisions (in which the participant must judge the relationship between the prime and the target, e.g., Hoppitt, Mathews, Yiend \& Mackintosh, 2010a) or lexical decisions (in which the participant must judge if the target is a word or a non-word e.g., Hoppitt et al., 2010b).

\section{Test Phases}

Following the training phase, a test phase is administered in order to assess the effect of training on interpretive biases. Test phases can be broadly categorised as either delayed or online tasks.

Delayed tasks. Delayed tasks are commonly, though not exclusively, used with scenario training, and typically involve an interval between the presentation 
of the ambiguous stimuli and the participant's response. This means that the participant must think back to their original interpretation of the scenario and report it, rather than produce it immediately after the onset of the ambiguous stimuli.

Recognition task. The recognition task is the most commonly used task in CBM-I studies to date. Developed by Mathews and Mackintosh (2002) this task involves a number of phases. After training, participants read ambiguous scenarios that may or may not involve solving a word fragment. The key here is that the scenario remains ambiguous. Then after a delay, participants are asked to rate the similarity of possible positive and negative interpretations of the critical scenarios in order to test whether an interpretive bias is present. Interpretive biases are reflected by greater endorsement of training-congruent interpretations of the critical scenarios. The underlying assumption is that participants will endorse the test sentence that most closely aligns with their original interpretation of the critical scenario.

The recognition task reliably measures interpretive biases in a negative and benign direction (e.g., Mackintosh et al., 2006; Mathews, Ridgeway, Cook \& Yiend, 2007). Beneficially, it tests the interpretation of highly descriptive ambiguous scenarios that describe realistic events, giving it some ecological validity. Furthermore, participants report not being aware of the purpose of this manipulation (Beard, 2011), attenuating the risk that demand characteristics drive task performance.

The ambiguous social situation interpretation questionnaire. The ASSIQ is a 24-item questionnaire designed to measure participants' interpretations of emotionally ambiguous events (Stopa \& Clark, 2000). Participants read short scenarios describing a mix of ambiguous social (e.g., You go into a shop and the assistant ignores you) and non-social situations (e.g., A letter marked 'urgent' arrives). Following a prompt of 'Why?' participants write down the first reason they can think of for why this event has occurred. Participants then read three potential explanations for this event (one negative and two benign) and rank them in terms of how likely it is that they would have thought of this explanation. This is considered a delayed task as participants are asked to compare the offered interpretations to their own original interpretation 
of the event. The higher the ranking of the negative interpretation the more negative the interpretation bias score.

Limitations of delayed tasks. Despite their frequent use in CBM-I paradigms, delayed tasks have some limitations that restrict their ability to directly assess interpretive biases or elucidate the cognitive processes that are recruited when participants make a response. Delayed tasks provide participants with a number of response options and do not place a limit on the time that a participant can view them or make a response. This means that a participant's final response reflects a great number of cognitive processes over and above their immediate interpretation of ambiguous stimuli. Their response may index their initial interpretation of the scenario; but responses could also reflect changes in interpretation that occurred during encoding of the scenario, during its storage and consolidation during the delay, or during the retrieval process at test. Because emotional biases are also evident in memory and decision processes (Blanchette \& Richards, 2010; Mathews, 2012; Salemink, Hertel, \& Mackintosh, 2010; Tran, Hertel, \& Joormann, 2011), a delayed similarity judgment cannot be taken as an indicator of interpretation per se.

Secondly, delayed tasks may not assess a participant's recollection of the critical scenario (and their interpretation of it), but their familiarity with the provided response options, which could be driven by the consistent use of one valence during the training phase. That is, the repeated presentation of negative information during a negative training phase may increase the likelihood that a participant endorses a negative over a benign response option. In this way a participant is simply responding in a manner congruent with the valence of the training phase and their responses do not reflect their actual interpretation (or even memory) of the original scenarios (Harvey, Watkins, Mansell \& Shafran, 2004).

Online tasks. An alternative test phase that has been used in CBM-I research is an 'online' task, which taps into a participant's interpretation of ambiguous stimuli at the time of presentation. Online tasks require participants to make judgments about stimuli as they are presented, so as to index interpretation as (or shortly after) it occurs. 
The extrinsic affective simon task. The EAST is an online task that requires participants to learn associations between direction keys and the emotionality and colour of word stimuli (Salemink, van den Hout \& Kindt, 2009). For example, participants may be taught to press the left key for a negative word and the right key for a benign word. They are also taught to press the left key for a blue word and the right key for a red word. Participants are therefore taught two response mappings for the left and right direction keys. Even when participants are told to focus only on the colour of the word, interference on response times to press a correct direction key can be observed depending on whether the stimuli are response congruent (e.g., a blue negative word) or response incongruent (e.g., a blue benign word). When a homograph is used in the colour judgement task, participants' response times can reflect the interpretation a participant has made. For example, if the homograph Fall is presented in blue ink, participants who have interpreted it in a negative manner may respond faster than participants who have interpreted it in a benign manner (as blue requires a left key press whilst a benign word requires a right key press). The EAST has been considered an implicit measure of interpretation, because the participant is never required to interpret the homograph explicitly.

Scrambled sentences task. Participants are presented with a scrambled sentence made from six randomly ordered words. One negative and one benign phrase can be created by using just five of the words e.g.,

"apprehensive people with relaxed new I'm".

A cognitive load is also introduced (to minimize the ability to re-interpret) by asking participants to remember a six-digit number while generating the sentence. An interpretation bias is inferred if participants report significantly more sentences of a particular valence following training. Both negative and benign interpretive biases have been demonstrated in healthy and anxious populations following interpretation training (Bowler et. al., 2012; Standage, Ashwin \& Fox, 2010).

Priming tasks. The most common online tasks use semantic priming to infer an emotional interpretive bias. In a typical (unambiguous) semantic priming task, a prime word is presented (e.g., Cat), followed by a target word to which the participant makes a response (e.g., lexical decision, naming, or judging 
its relatedness to the prime). If the prime is related to the target (e.g., Dog), responses are facilitated (that is, they are faster and/or more accurate) relative to unrelated primes (e.g., Kayak). Priming is thought to occur through spreading activation from the meaning of the prime to the meaning of the target (Schvaneveldt \& Meyer, 1973). When the prime word is an emotionally ambiguous homograph (e.g., Terminal) it can be related to both negative targets (e.g., Cancer) and benign targets (e.g., Airport). Participants use some combination of meaning frequency (or likelihood) and context to select a single meaning (e.g., Swinney, 1979; Simpson \& Burgess, 1985). Participant's responses should therefore be facilitated to targets that are consistent with their interpretation of the ambiguous prime. CBM-I uses this task to examine the influence of the training condition on the accessibility (or availability) of alternative meanings during the test phase. If training influences the relative availability of different meanings of an ambiguous word, then participants should show facilitation for judgments involving targets that are congruent with their training valence. Priming tasks in CBM-I typically show training effects (e.g., Grey and Mathews, 2000) whereby participants are faster to make responses to training-congruent than to training-incongruent meanings.

Mixing Training and Test Phases. A potential concern with the tasks used in CBM-I research is the methodological overlap between training and test phases. Most scenario-based training uses a critical scenario recognition test phase and most word-based training phases are coupled with word-based online tasks.

It is possible that, because the task demands of training and test are so similar, participants' performance simply reflects the adoption of a task-specific strategy or task-related expertise rather than an actual change in interpretation processes (Saleminck, van den Hout \& Kindt, 2010). One finding which speaks to this possibility is the failure to find interpretation change when using training and test tasks with less overlap. For example, interpretive biases have not been observed in the delayed ASSIQ or the online EAST (Extrinsic Affective Simon Task) tasks following Mathews and Mackintosh's scenario-based training task (Salemink, van den Hout \& Kindt, 2007; Salemink, van den Hout \& Kindt, 2009).

Overall, the interpretive biases that are observed following training may 
be due to a change in the interpretation of emotional ambiguity. But alternatively they may reflect memory or decision biases at test caused by a delayed test phase, or enhanced performance on a particular task due to methodological overlap between training and test. Importantly, the choice of training and test procedures can limit the conclusions that can be drawn about the mechanisms that produce interpretive change.

\section{Ambiguity Resolution in Non-Emotional Contexts}

CBM-I research has largely focused on the applications of this paradigm as a potential treatment for clinical disorders. As a result, previous research has, for the most part, not focused on the mechanisms of interpretation change, and has not always been informed by cognitive or psycholinguistic research on interpretation processes. This focus has led to the use of CBM-I paradigms that are not always well controlled against demand characteristics, and reliance on stimuli that may not be piloted or balanced on psycholinguistic variables that can themselves bias interpretation. Furthermore, methodological issues of procedural overlap have not been previously addressed. These factors are all critical if we are to understand the mechanisms by which we come to interpret an emotional event. This is where a psycholinguistic and cognitive approach is relevant for CBM-I research. There is a strong tradition of psycholinguistic research on ambiguity resolution that can inform research methods and theoretical approaches for CBM-I studies. In this section, I discuss the current state of lexical ambiguity research and detail where this research may be useful in addressing some of the remaining questions around CBM-I.

Semantic network models are commonly used to describe the organisation of semantic information within the mental lexicon (e.g., Balota, 1994; Collins \& Loftus, 1975; Kawamoto, 1993; Posner \& Snyder, 1975; Seidenberg \& MeClelland, 1989). In a typical semantic network, words and their associated features and characteristics are assigned nodes within the semantic system. These nodes are connected in terms of their learned associations and the distance between each node reflects the strength of its association with that particular concept (e.g., Bird-Wings is more closely associated than Bat-Wings). The organisation of this information affects retrieval processes and in turn impacts response times 
(Carroll \& White, 1973). The semantic network theory is able to account for priming effects that are regularly seen in behavioural experiments such as the finding that concepts prime conceptually-related meanings. All nodes are assumed to have a resting level of activation that is enhanced when primed by a related concept. Therefore in a lexical decision task participants are faster to say "bird" is a word when preceded by the related prime "wings" than by the unrelated prime "nurse" (Meyer \& Schvaneveldt, 1971; Neely, 1976, 1977)

There are a number of steps involved in word recognition (for a review see Balota, Yap \& Cortese, 2006; Frauenfelder \& Komisarjevsky Tyler, 1984), which span the initial reception of a signal to final access to a semantic representation. When an information signal, such as a spoken or written word, is detected by the system a cascade of processes begins, starting with activation. Lexical (word-level) and semantic (conceptual level) information both contribute to the activation and subsequent selection of a single meaning for any given input signal. Activation is the process by which entries in the mental lexicon are matched for physical similarity with the lexical input; a number of lexical models suggest ways in which this may occur, (e.g. the cohort model, Marslen-Wilson, 1987; the connectionist model, Seidenberg \& McClelland, 1989; the logogen model, Morton, 1969; the autonomous search mode, Forster, 1976). Typically, the features of a word form are compared to a number of similarly formed representations stored within a 'mental dictionary'. The number of competing words that are activated affects the speed with which a word is matched to its correct representation (Marslen-Wilson, 1987; Tyler, 1984). After appropriate entries have been activated, word recognition occurs when one entry (and its corresponding meaning) receives enough evidence in its favour that it is selected. Debate still exists regarding the specific mechanisms of recognition, for example on the number of intermediate processes involved (e.g., the existence of a 'lemma' level, Caramazza, 1997), the timing with which the word form passes through lexical and semantic stages, the processes that are considered characteristic of different stages (e.g., pure orthographic activation in lexical access vs. orthographic effects combined with word frequency effects) or even whether these levels are independent or interactive. However, there is a general consensus across these models that the lexical and semantic levels constitute 
different stages and that they are likely to be accessed in a systematic order. In this thesis I don't distinguish between specific models, but experiments and predictions are driven by the basic two-stage process of meaning activation and meaning selection that are common to many models.

\section{Models of Meaning Sense Disambiguation}

The activation and selection of meanings within the mental lexicon is even more complex when the incoming signal has more than one possible interpretation. For a number of years there was a significant modularity debate within the semantic ambiguity literature regarding the relative independence of lexical activation and selection processes (Gorfein \& Bubka, 1989; Simpson \& Burgess, 1989, Simpson, 1994). The debate raised the question of whether activation of ambiguous word meanings could be affected by contextual effects, in order to guide the selection of an appropriate meaning. According to selective access accounts, activation and selection processes happened at the same time and therefore only the contextually appropriate meanings of an ambiguous word was activated (e.g., Schvaneveldt, Meyer, \& Becker, 1976). Exhaustive access accounts on the other hand proposed that activation and selection represented different stages so that initial activation occurs for all meanings of an ambiguous word and selection is then driven by contextual information (Swinney, 1979; Tanenhaus, Leiman, \& Seidenberg, 1979). Research to date shows support for a non-modular and context sensitive account of ambiguity resolution that falls somewhere between selective access and exhaustive access accounts (e.g., the Reordered Access Model, Duffy, Morris \& Rayner, 1988). These models propose that factors such as meaning frequency and context interact in order to account for activation and selection patterns in meaning sense disambiguation. Selection must necessarily occur as more than one meaning of an ambiguous word will be activated; however, early activation patterns can be influenced to some extent by some contextual information.

One contemporary model of semantic ambiguity resolution is the Independent Activation Model developed by Twilley and Dixon (2000). The model considers lexical and semantic levels of analysis to contribute independently and in parallel to the disambiguation of homographs. In their account, Twilley and Dixon (2000) model activation from both an orthographic input (early lexical- 
level information such as form and frequency) and an integration input (late semantic-level information such as the surrounding sentential context and other sources of priming). From here selection occurs once activation for each meaning is summed and an appropriate word can be integrated into the surrounding context. The interpretation of ambiguity involves three broad stages from the onset of a stimulus to the final response of the participant. First, its perceptual features are mapped onto stored lexical representations, activating all possible meaning candidates. Following this, contextual integration occurs via consideration of surrounding context and knowledge, resulting in selection of a single meaning. Finally, post-lexical processes - such as a participant's reappraisal of their initial interpretation - occur before the participant reports their chosen meaning. Both the Reordered Access model and the Independent Activation model account for activation and selection patterns in meaning sense disambiguation and highlight the independent and parallel contributions a word receives from context and meaning frequency.

The selection process. Homographs - written words that have more than one possible interpretation (e.g., Terminal) - are ideal stimuli to use in studies of ambiguous word recognition. They have the benefit that a number of factors such as length, pronunciation and orthographic form are held constant, while still allowing for access to two separate meanings. When a word has more than one possible meaning we rely on a number of factors to disambiguate it. One such factor is context - we select the meaning that best "fits" the sentence. Another factor is meaning frequency - we choose meanings that are more common and therefore more likely.

Context Effects. Context has been established as an important factor in ambiguous word meaning activation and selection (Binder \& Morris, 1995; Onifer \& Swinney, 1981; Simpson, 1984; Swinney, 1979; Tanenhaus et al., 1979; Schvaneveldt \& Meyer, 1976). Context acts at all levels of word recognition, from facilitation of perceptual recognition of lexical stimuli to the integration of an individual word into a wider sentential discourse (Masson \& Borowsky, 1998). Context can also act as a source of information in either a local or a global manner. Local context refers to information that is presented in close proximity to the target word and can be manipulated trial-by-trial. The most well-known 
example of a local context effects on word recognition is the semantic priming effect (e.g., Meyer \& Schvaneveldt, 1971; Neely, 1976, 1977) in which primes facilitate responses to semantically related target words. This effect reflects automatic spreading activation to related concepts within the semantic network which speeds lexical access and word recognition for semantically related words.

On the other hand, global context acts at a broader level and "sets the scene" by presenting information prior to the presentation of the target word (e.g., Kambe, Rayner \& Duffy, 2001; Wiley \& Rayner, 2000). One study, using unambiguous words, manipulated global and local context in a study that represented natural discourse processing (Hess, Foss \& Carroll, 1995). During the course of the experiment participants heard long passages and were required to name the final word in the sentence. In one condition the passage reflected a global context in support of the final word, yet the local context was unrelated to the target e.g.,

The computer science major met a woman who he was very fond of. He had admired her for a while but was not sure how to express himself. He always got nervous when trying to express himself verbally so the computer science major wrote the poem.

In a second condition participants heard passages with a congruent local context prior to the target set in an incongruent global context e.g.,

The English major was taking a computer science class that she was struggling with. There was a big project that was due at the end of the semester which she had put off doing. Finally, last weekend the English major wrote the

poem.

The facilitation of naming times was only observed when the target was related to the global context, regardless of whether the local context was congruent or incongruent with the target. In this way global context appears to build up a general conceptual representation of the scenario at a semantic rather than a lexical level. 
In one of the earliest studies of the effects of global context on ambiguity resolution, Swinney (1979) examined the time course of meaning activation and selection from ambiguous words embedded in sentential contexts. Participants heard sentences containing a homophone (a spoken word with more than one possible interpretation) and performed a lexical decision to a visual word that was related to one of the meanings of the ambiguous word e.g.,

Rumour had it that, for years, the government building had been plagued with problems. The man was not surprised when he found several spiders, roaches and other bugs in the corner of the room.

Note that the sentence provides context for interpretation of the ambiguous word "bugs". Following the onset of the homophone prime bugs, participants saw a related target word e.g., ant or spy, an unrelated word e.g., sew, or a non-word. The time between the onset of the homophone prime and the target (Stimulus Onset Asynchrony: SOA) was manipulated to determine when meanings became available and when they were selected. Regardless of whether the global context surrounding the word biased one particular meaning of the ambiguous word or was neutral, participants initially (within 250ms of stimulus onset) showed facilitated responses to targets associated with all possible meanings of an ambiguous word. However, after a delay (750-1000ms after stimulus onset) participants' responses were only facilitated for the contextuallyappropriate meaning (e.g., ant, which is consistent with the context provided by “spiders, roaches and other..."). Swinney's (1979) findings are consistent with dual stage models of ambiguity resolution (e.g., Twilley \& Dixon, 2000), which propose that when an ambiguous word is detected all possible meanings first become activated, and context then guides selection processes at a later stage.

Meaning Frequency Effects. Research conducted with ambiguous words presented in isolation shows that the initial activation of alternative meanings is driven by the relative frequency of each meaning. Following Swinney's work, Simpson and Burgess (1985) examined how ambiguity resolution occurred in the absence of any context. In a semantic priming task, participants performed lexical decisions to targets related to the dominant and subordinate meanings of 
ambiguous primes (e.g., Bank-Money or Bank-River). The SOA was manipulated in order to examine the time course of the availability of different meanings of the ambiguous prime. At a very short SOA $(<300 \mathrm{~ms})$ only the dominant meaning of ambiguous words received any activation (i.e., lexical decisions were faster for Money than River. At a longer SOA (300ms) equivalent activation was observed for both dominant and subordinate meanings, in that participants were equally as fast to make lexical decisions to both types of word targets. However, at a much longer SOA (700ms), the activation of the dominant meaning was maintained whilst activation for the subordinate meaning decreased. After $750 \mathrm{~ms}$ no activation of the subordinate meaning was observed. These findings support the idea that meaning activation and selection in the absence of context occurs automatically in a manner dictated by meaning frequency (Twilley \& Dixon, 2000). Much discussion has focused on the fate of the subordinate meaning, and at this time there is debate as to whether the reduction in activation of the subordinate meaning is due to decay, mutual inhibition from the dominant meaning or a different process altogether (see Gernsbacher, Varner, \& Faust, 1990; Gernsbacher \& St. John, 2001; MacLeod, Dodd, Sheard, Wilson, \& Bibi, 2003; McNamara \& McDaniel, 2004, Simpson, 1984 \& Simpson \& Kang, 1994, for a detailed discussion regarding the controversies surrounding the concept of active suppression).

Automatic and controlled processing. Taken together, these findings suggest that the process of arriving at a single meaning is driven by an interaction between the relative dominance of meaning candidates (an "automatic" process) and the preceding context (a "controlled" process). In the literature we can see the evidence for two stages in ambiguity resolution, an early stage, in which all possible meanings of an ambiguous word become activated, and a later stage, in which context and frequency are used to settle upon one meaning and to reject all other meanings. This is in line with common models of ambiguous word recognition such as context-sensitive, exhaustive access accounts of lexical access (the Reordered Access Model; Duffy et al., 1988) and Twilley \& Dixon's (2000) Independent Activation Model.

Post-lexical processes. Following meaning selection, a participant still needs to make a response to the target. Processes that occur between selection 
and response are broadly referred to as post-lexical processes. Post-lexical effects commonly occur at the response stage when a participant initiates an action in accordance with the task demands, e.g., pressing a button in a lexical decision task (Coulson \& Federmeier, in press). A participant's response can be affected post-lexically by the demands of the task in two ways. First, introducing a considerable delay between meaning selection and the required response allows decision processes to come into play. For example, a delay may give the participant time to reinterpret the ambiguous word or to report a meaning that is consistent with their expectations of the task's purpose. This more "controlled" process of acting in accordance with expectations takes time. Therefore, without time pressure on a task (e.g., in homophone spelling tasks) a participant's response may not reflect their initial interpretation of an ambiguous word, even in an "online" task. Post-lexical factors are an important consideration when developing a CBM-I paradigm.

Second, even without a delay between stimulus presentation and response, the type of task that a participant is required to perform can affect the way in which they respond. This is particularly relevant in priming tasks. For example, in a naming task a participant is required to articulate the target word after the presentation of a prime word. While the prime can facilitate the speed with which participants name targets, success on the task does not rely on interpretation of the prime; participants need only access the phonemic sequence for the target, (which might or might not be influenced by its semantic representation). Alternatively, the use of a semantic judgment task, in which a participant must decide whether the target is conceptually related to the prime, forces the participant to read and interpret the meaning of each word in order to make a correct decision. Thus, after lexical access has occurred, the task that a person is required to perform can affect the cognitive processes that are recruited and the speed with which they proceed. For example, the semantic congruity of information preceding a word target (either at a sentence or a single word level) facilitates target lexical decision times but not target naming latencies (de Groot, 1984; West \& Stanovich, 1984). Therefore it is important to consider what task is used in a test phase especially when the interpretation of ambiguity is assessed. 


\section{Meaning Disambiguation in Emotional Contexts}

Sometimes, the ambiguity in a word is related to its emotional quality (i.e., words with one valenced and one benign interpretation). There are a number of ways emotion could influence interpretation processes. Psycholinguistic models of emotional word recognition suggest that words are stored in dynamic networks that reflect links between the word, its features and its emotional connotations (e.g., Bower, 1981; Barsalou, 1999). It has also been suggested that emotional words may link to broader information structures such as emotionspecific motor representations and physiological responses (e.g., Lang, 1994). Although all models propose the existence of lexical and semantic levels of processing, there is little consensus as to what characterises each level. Therefore there is no agreement on whether emotion can be considered a lexical or a semantic feature (or perhaps both; Palazova, 2014). In fact, effects of emotion can be observed at all stages of word recognition - from initial perceptual processing through to post-lexical stages (e.g., Kissler, Assadollahi \& Herbert, 2006; Kissler \& Herbert, 2013). Lexical access is faster for emotional compared to neutral words suggesting that valence and/or arousal facilitates the speed of early word activation (e.g., Kissler \& Herbert, 2013). Furthermore, exposure to a prior emotional valence appears to aid the later integration of similarly valenced words, suggesting emotion works to guide the selection of meanings (e.g., Schact \& Sommer, 2009). In this way emotion appears to broadly impact both lexical and semantic levels of word recognition.

Meaning selection can be affected by emotional features that are inherent in the word form itself or by factors associated with the interpreter. Generally speaking, word form effects have been examined within psycholinguistic and cognitive psychology domains, whilst individual differences in emotional ambiguity resolution have been of more interest to applied clinical psychology researchers.

One of the most well-studied effects of emotion on word recognition is the affective priming effect. Affective (or emotional) priming is observed when prior emotional context facilitates the processing of similarly valenced stimuli. In regards to lexical context, the presence of emotional words (e.g., Death) facilitates later processing of similarly valenced information (e.g., Aggressive) 
compared to neutral words (e.g., Rose; Klauer, 2011). Emotional priming effects can be considered automatic in the sense that emotional priming is efficient, not dependent on processing resources (Hermans, Crombez, \& Eelen, 2000), observed at relatively short SOAs (De Houer, Heermans \& Eelen, 1998; Fazio, Sanbonmatsu, Powell, \& Kardes 1986) and does not require awareness of the prime (Draine \& Greenwald, 1998). In this sense, emotional priming behaves like a fast-acting but short-lived context, which facilitates responses to similarly valenced information in an efficient and non-discriminatory manner.

Emotional experience can also bias the access of meanings in an emotioncongruent manner. The mood-as-information theory proposes that the positive or negative feelings associated with emotions, mood states and physiological experiences act as sources of information which people use to interpret their surroundings (this is not assumed to be a conscious process; Schwarz \& Clore, 2011). While not a linguistic model, this theory is consistent with psycholinguistic research, which suggest that induced mood states can bias the interpretation of emotional ambiguity. People are faster to make interpretations of emotional ambiguity in a direction consistent with their own mood, suggesting that experienced mood states can serve as a source of bias in emotional interpretation (Clark, Teasdale, Broadbent \& Martin, 1983; Challis \& Krane, 1988; Halberstadt et al., 1995).

In these two ways emotion appears to act as a prior context, which drives the activation and/or selection of one meaning from emotionally ambiguous information. This is in line with Bower's Associative Semantic Network Model (1981) in which emotion is assigned a node in a semantic network just like any other piece of semantic information. In this model positive feedback loops occur between negative mood states and negative information processing (such as negative memories) that then serve to reinforce the mood state and enhance further activation of mood-congruent stimuli.

\section{Candidate Mechanisms}

It is possible to compare and contrast mechanistic explanations for modified interpretive biases by considering what is known about the processes involved in emotional ambiguity resolution. Two accounts have been raised that stand as real candidates for the effects underlying interpretive change - an 
Emotional Priming Account and an Ambiguity Resolution Account. According to an Emotional Priming Account the activation of an emotional category during training increases the "ease" of activation or selection of meanings related to that particular emotional category, resulting in facilitated performance for similarly valenced information at a later stage (Grey \& Mathews, 2000). This is believed to occur through a process similar to that of spreading activation seen in early semantic priming effects and proposed by semantic network models (e.g., Neely, 1967). In an update to the conventional semantic priming model, Becker, Moscovitch, Behrmann and Joordens (1997) propose that the prior activation of a particular word meaning deepens an attractor basin. The activated basin increases its 'pull' and the probability that the same meaning will receive activation upon further presentations. The deepening of this basin also increases the spread of activation to conceptually similar meanings, therefore providing a benefit to meanings similar to the primed meaning. According to this account, the repeated resolution of ambiguity is not an important feature of the training phase; rather the repeated exposure to negative information is responsible for inducing a negative interpretive bias.

Alternatively, according to an Ambiguity Resolution Account, training induces the use of an implicit production rule that biases selection towards emotionally congruent meanings under conditions of ambiguity. According to this view, the resolution of ambiguity is the critical feature of the training paradigm. A learned production rule is then developed over time, similar to learned production rules used in problem solving (e.g., IF there is ambiguity THEN select the 'negative'/'benign' meaning). In this case, the repeated presentation of a particular valence during training is not sufficient to shift an interpretation bias; rather, the process of disambiguation itself becomes biased, and therefore interpretive bias modification only occurs when the training requires ambiguity resolution.

Based on current models of ambiguity resolution (Duffy et al., 1988; Twilley \& Dixon, 2000), there are three processing stages at which training could alter interpretation. First, training may serve to bias the relative levels of early activation that each meaning receives, directly affecting the likelihood of selection of one meaning over another. In line with an Emotional Priming 
Account, training may increase the activation of all words associated with the training valence, making the activation and selection of emotionally-congruent meanings more likely. Second, training may induce an implicit production rule, which directly contributes to meaning selection at a later stage. In this way training could act similarly to a procedural context; increasing the likelihood of selection of a particular valence in the presence of ambiguity (e.g., in the case of negative training, a participant may learn that an ambiguous context signals a subsequent negative interpretation). Finally, training may not directly affect the immediate interpretation of emotionally ambiguous stimuli at all. Instead the changes in measures of interpretation bias following training may reflect a number of other cognitive processes such as a participants' reinterpretation of emotional ambiguity, emotional biases in memory, or their acting in accord with their awareness of the task's purpose. Importantly, these accounts are not mutually exclusive - training could conceivably facilitate changes at any or all stages of meaning selection.

\section{Overview of the Thesis}

An important review by Blanchette and Richards (2010) addressed the effects of emotion on higher order cognitive processes. Of particular interest is their discussion of the mechanisms underlying interpretive biases. Their discussion raised two questions, namely 1) At what stage is interpretation affected by emotional context i.e., the activation, selection or (post-lexical) response stage and 2) what is the causal direction between interpretive biases and emotional effects such as state mood changes.

CBM-I research has made important contributions to the second question - understanding the relationship between psychopathology and cognitive biases by examining the directionality of their interactions and identifying mediating variables. However, little research has focused on elucidating the cognitive processes that are affected in lexical ambiguity resolution following CBM-I training (MacLeod, Koster, \& Fox, 2009). Therefore, the overarching aim of this thesis is to identify and test possible mechanisms for the observed changes in interpretive biases following training. I focus on changes in interpretive biases in healthy populations in order to minimise individual differences associated with psychopathology that can also affect interpretation. 
Chapter 2 describes the development and validation of a novel CBM-I task that remedies some of the limitations identified in previous research. An online task was used at test because of its advantages in minimising post-lexical effects, paired with a scenario-based training phase to minimise procedural overlap. A primed relatedness judgement task was used in the test phase in order to force selection of one meaning from an ambiguous prime. The experiment described in Chapter 3 tests an Emotional Priming Account of interpretive bias modification by determining whether training affects the activation of valenced meanings available at shorter SOAs. Chapter 4 describes two experiments is which the CBM-I task is systematically modified in order to address the Ambiguity Resolution Account; by manipulating the requirement for ambiguity resolution during the training phase. In Chapter 5 I use a primed lexical decision in the test phase to determine whether training effects generalise to a task that does not require active interpretation of the prime. Chapter 6 summarises the contribution of my research, integrates my findings with models of ambiguity resolution and emotional interpretation, and identifies implications for models of cognitive bias and future directions for CBM-I research. 


\section{Chapter 2: Developing and Validating an Interpretive Bias Modification}

\section{Paradigm}

Three key methodological concerns were considered in the development of the paradigm used in this thesis. Firstly, criticisms have been levelled at CBM-I studies for the procedural overlap between training and test phases. Overlap makes it difficult to assess whether performance in the test phase is due to actual interpretation change, post-lexical processes, or participants' developed expertise at a particular component of the task. Secondly, the delayed test phases commonly used in CBM-I tasks are limited in their ability to inform us about interpretive processes as they occur. It is quite possible that interpretation changes observed on delayed tasks reflect other mediating cognitive processes or response biases. Finally, stimuli used in training and test paradigms in the past have received little piloting to control for orthographic features known to impact lexical access and the selection of meanings. I therefore extensively piloted the stimuli that were ultimately selected for use in further studies.

For these reasons, an ambiguous scenario training phase and a semantic priming task at test were selected. The training phase was modelled on previous scenario-based training phases, which reliably modify interpretive biases, with one key difference. In previous scenario-based training phases the word fragment is typically a sentence-final word and completion of the fragment constrains the interpretation of the scenario (e.g., Mathews \& Mackintosh, 2000). This means that the training-appropriate interpretation of the scenario was provided for the participants when they completed the word fragment. In the current paradigm, word fragments were simply negative and benign words that were associated with possible interpretations a participant could make from the scenarios. In this way, participants were not required to interpret the scenarios in a specific way in order to correctly solve word fragments; however, efficient fragment completion should be facilitated by consistent interpretation of the scenarios. It was thought that such unconstrained (but guided) interpretation might encourage more active interpretation than the more passive task that is traditionally used when the experimenter provides the interpretation. Importantly, the training still contained the common features of scenario-based training including emotionally ambiguous scenes, semantically "rich" 
descriptions of events, and valenced emotional word fragments.

In the test phase a semantic priming task was selected. Priming tasks are most commonly used to assess the access of homograph meanings in language studies (Twilley \& Dixon, 1999). Word recognition is most commonly studied with the classic semantic priming task (Neely, 1977). It is possible to manipulate many aspects of the priming task to identify factors that affect different stages of word recognition. For example it is possible to manipulate the duration of the prime, the delay between the prime and the target, and the task that the participant is required to perform (e.g., lexical decision or relatedness judgement). These manipulations can be used to determine the effects of training on specific interpretation processes, the time course of meaning activation and the effects of context on meaning selection.

The priming task used to test interpretation in the first four experiments was a semantic relationship judgment adapted from Richards and French (1992). Participants saw emotionally ambiguous homograph primes (e.g., Terminal) followed after a short delay (750ms) by negative (e.g., Cancer) or benign (e.g., Airport) related targets or negative (e.g., Lonely) or benign (e.g., Table) unrelated targets. A sufficient delay after the presentation of the prime allows for both the activation and selection of its meaning before the onset of the target. Participants then judged whether the target was related or unrelated to the prime. The speed of this response provides an indication of the selected meaning of the prime. For example, if a participant selects the transportation-related meaning of Terminal, when the target word Airport is presented they will be faster to judge the prime and target as related compared to when the target word Cancer is presented. The relatedness task provides direct and online assessment of interpretive biases, and has considerably different task demands to the word fragment completion task in the training phase, minimizing procedural overlap between training and test. A relationship decision task was chosen over the more common lexical decision task in the first four experiments in order to force participants to interpret the prime word (Balota \& Paul, 1996). However, a lexical decision task was used in Experiment 5 for comparison purposes. 


\section{Task Development}

\section{Stimuli and Apparatus}

In all experiments, stimuli were presented using a Dell PC computer running Psychology Software Tools' E-Prime Suite version 2.0 (Schneider, Eschman \& Zuccolotto, 2002). Stimuli were presented centrally on a 23-inch Alienware 2310 monitor with a vertical refresh rate of $60 \mathrm{~Hz}$. Sentences and words were presented centrally with Calibri 18 pt font, in lower case black letters on a white background. Participants were seated at a viewing distance of approximately $60 \mathrm{~cm}$. All participants were testing individually in a private testing room unless otherwise noted.

\section{Training Phase}

During training, participants read emotionally ambiguous sentences followed by a related negative or benign fragment (depending on assigned training condition; See Figure 4.). The sentences and words used during training were constructed and piloted to ensure the stimuli did not vary on basic orthographic properties as outlined below.

Sentences. Seventy-nine emotionally ambiguous, self-referential sentences were constructed that had one negative and one benign interpretation (e. g., The dog is running towards you; The boss wants to see you; See Appendix A for all training sentences.) The sentences were adapted from Mathews and Mackintosh (2000) and Mogg, Bradbury, and Bradley (2006), with additional sentences generated by our research team. The sentences were piloted on nine people to remove badly constructed sentences and those that were strongly biased towards one particular interpretation. Pilot participants were asked to report the first three words that came into their mind after reading each sentence. Initial responses made to each sentence and the overall proportion of negative and neutral responses to each sentence were calculated. Sentences that consistently produced (over $65 \%$ of the time) negative or neutral associates were removed. This left 45 sentences for which pilot participants spontaneously generated both negative and benign associates.

Word Fragments. In the training phase, each ambiguous sentence was followed by a word fragment to be completed. Fragments were consistently 
related to either a negative or a benign interpretation of the sentence, depending on training condition. One negative and one neutral associate were selected from the most commonly produced responses generated during piloting of the test sentences. These critical words became the fragments used in training

During CBM-I training, participants saw different fragments in the two training conditions. Therefore, it was important to ensure that the two groups of critical words (used to create the fragments) did not significantly differ on core orthographic features (See Table 1.). This ensured that any change in interpretation in the test phase could be attributed to the training condition, and not the specific word fragments that were used in each condition. Independent $t$ tests revealed that the two sets of fragment words did not significantly differ in length, frequency, orthographic neighbourhood size or orthographic frequency (normative data collected from Wilson, 1988 and Medler \& Binder, 2005).

\section{Table 1}

Mean orthographic properties of negative and neutral sentence associates.

\begin{tabular}{lcccc}
\hline & \multicolumn{2}{c}{ Negative Targets } & \multicolumn{2}{c}{ Benign Targets } \\
& Mean & SD & Mean & SD \\
\hline Length & 6.89 & 2.12 & 6.91 & 1.96 \\
Frequency & 95.13 & 55.33 & 62.73 & 76.23 \\
Orthographic Frequency & 10.48 & 24.18 & 15.40 & 5.79 \\
Orthographic Neighbourhood & 2.64 & 3.77 & 2.87 & 5.01 \\
\hline
\end{tabular}

Note: Orthographic Neighbourhood is the number of words (of the same length) that differ by only one letter. Frequency refers to how often a word is encountered per one million presentations of text (Kucera \& Francis norms, 1967)

Critical words need not necessarily be valenced themselves in order to be related to a valenced interpretation of the sentence, but they generally were. Valence ratings for the fragments were collected from a large corpus of affective norms (Warriner, Kuperman \& Brysbaert, 2013) and independent samples ttests confirmed that critical words used as fragments in the negative training phase were significantly more negative $(M=2.87, S D=0.86)$ than the benign fragments used in benign training $(M=6.61, S D=0.187), t(86)=16.37, p<$. 
001. Critical words were transformed into fragments by removing a consonant and a vowel from each word (only one letter was removed for the shorter word fragments). The word fragments were then piloted to ensure that fragments used in the negative and benign training conditions did not differ in completion difficulty.

Seventeen pilot participants saw each training sentence once; half the sentences were paired with their negative word fragments and half with their benign word fragments. Participants pressed the spacebar to indicate that they had solved the word fragment and then entered their response. Ease of completion was operationalised as the time taken to press the spacebar after the onset of the word fragment for correct solutions. Independent samples t-tests confirmed that there were no significant differences in time taken $t(88)=1.36, p$ $=.18$, or accuracy $t(88)=0.84, p=.48$, in solving negative or benign word fragments (See Table 2.). The average time taken to solve a word fragment was $2121 \mathrm{~ms}(\mathrm{SD}=813 \mathrm{~ms})$ and accuracy was $96.69 \%(\mathrm{SD}=10.31)$.

\section{Test Phase}

The test phase consisted of a semantic priming task in which emotionally ambiguous primes were paired with targets related to their negative or benign meanings (See Figure 4.). Participants were required to make an explicit judgment about the semantic relationship between the prime and the target.

\section{Table 2}

Accuracy and time taken to complete word fragments as a function of target valence (Negative and Benign).

\begin{tabular}{lcccc}
\hline & \multicolumn{2}{c}{ Negative Associates } & \multicolumn{2}{c}{ Benign Associates } \\
& Mean & SD & Mean & SD \\
\hline Accuracy (\%) & 95.43 & 10.91 & 97.87 & 9.62 \\
Response Time (ms) & 2363 & 2952 & 1943 & 2250 \\
\hline
\end{tabular}

Homograph primes. For the test phase 143 homographs (e.g., patient) were collected which had one negative (e.g., hospital) and one benign meaning (e.g., calm). The homographs were balanced for meaning dominance based on a number of sources (Gawlick-Grendell \& Woltz, 1994; Gee \& Harris, 2010; Gorfein, 
Viviani \& Leddo, 1982; Twilley, Dixon, Taylor \& Clark, 1994) and piloted on six participants in order to norm for modern meaning additions (e.g., the ambiguous word cell was removed from the corpus because of the additional modern interpretation of cellphone) and New Zealand colloquialisms. Pilot participants were presented with lists containing ambiguous and unambiguous words to reduce the salience of ambiguity). They were asked to report the first three meanings that came to mind and a score was calculated for each word that reflected the proportion of responses produced for each meaning. Words that produced a consistent meaning (over two-thirds of the time) were discarded as unbalanced homographs. After exclusions, 52 balanced homographs remained that were used as primes.

Associated targets. Homograph primes were paired with related negative and benign target words that were gathered from the University of South Florida Free Association Norms (Nelson, McEvoy, Walling \& Wheeler, 1980.). Five pilot participants generated associates to each ambiguous prime to ensure that they were similarly ambiguous for native speakers of modern New Zealand English. Independent samples t-tests confirmed that negative and benign targets did not differ in length $t(88)=0.05, p=.96$ or word frequency $t(88)=1.18, p=.24$. Valence ratings were obtained from the affective norms database for each target (Warriner et al., 2013; Bradley \& Lang, 1999). Note that target words did not necessarily need to be valenced themselves to be related to the valenced meaning of an ambiguous word (e.g., fall - over) however independent t-tests confirmed that negative targets were rated as significantly more negative $(M=3.48, S D=1.18)$ than benign targets $(M=6.39, S D=.98), t$ $(102)=13.63, p<.001$. Homographs were also paired with one unrelated negative word and one unrelated benign word. Unrelated targets were created by rematching existing targets with primes to produce pairs that were no longer associated. Two lists were constructed (See Appendix C) for the purposes of counterbalancing. Meaning dominance was counterbalanced across lists as equally as possible. Each list contained the homograph twice, once paired with a related target and once paired with an unrelated target. If the target in the related pair was negative then the target in the unrelated pair was benign and vice versa (see Table 3 for an example using the prime stalk). Independent t-tests 
confirmed that there were no significant differences across lists in length, frequency, orthographic neighbourhood and orthographic frequency (the averaged frequency of the of the orthographic neighbours) target words (See Table 4).

\section{Table 3}

Typical allocation of primes and target valences across participants.

\begin{tabular}{ccccc}
\hline & Prime & Relationship & Valence & Target \\
\cline { 2 - 4 } Participant 1 & \multirow{2}{*}{ stalk } & Related & Negative & Creep \\
& & Unrelated & Benign & Kayak \\
\cline { 3 - 4 } Participant 2 & stalk & Related & Benign & Flower \\
& & Unrelated & Negative & Lonely \\
\hline
\end{tabular}

\section{Table 4}

Orthographic properties of targets for each counterbalanced list of homographs for the test phase.

\begin{tabular}{lcccc}
\hline & \multicolumn{2}{c}{ List 1 } & \multicolumn{2}{c}{ List 2 } \\
& Mean & SD & Mean & SD \\
\hline Length & 5.70 & 1.81 & 5.26 & 1.66 \\
Frequency & 58.8 & 185.10 & 74.38 & 208.39 \\
Orthographic Frequency & 4.42 & 4.12 & 5.48 & 5.70 \\
Orthographic Neighbourhood & 37.61 & 151.65 & 53.27 & 175.67 \\
\hline
\end{tabular}

Note: Orthographic Neighbourhood is the number of words (of the same length) that differ by only one letter. Frequency and Orthographic Frequency refers to how often a word is encountered per one million presentations of text. 

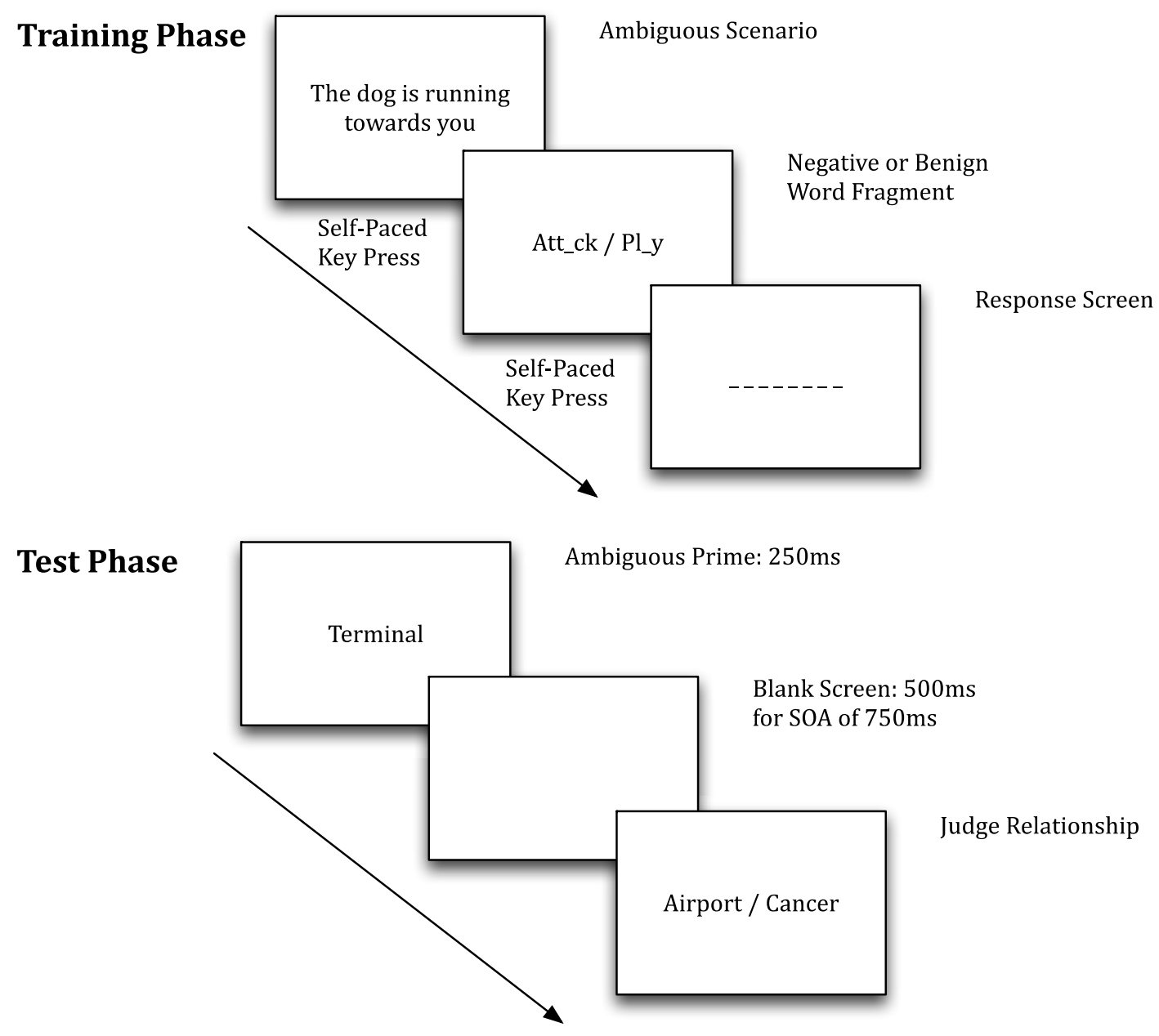

Figure 4. Novel CBM-I Procedure developed for Experiment 1.

During training, participants were assigned to read 45 emotionally ambiguous scenarios paired with either all negative or all benign word fragments. During the test phase, participants saw 52 emotionally ambiguous prime-target pairs, 26 targets were related to the benign meaning and 26 were related to the negative meaning. 


\section{Experiment 1a}

The purpose of Experiment 1a was to ensure that the novel training paradigm produced the expected interpretation bias as assessed with the semantic priming task. During training, participants read ambiguous sentences followed by negative or benign disambiguating word fragments. In the test phase, participants judged the relatedness of word pairs that consisted of an ambiguous prime followed by a target that was related to either the prime's negative or benign meaning. The relatively long SOA (750 ms) allowed time for both activation and selection of the meaning of the prime to occur before the onset of the target (e.g., Neely, 1977; Swinney, 1979). If the CBM-I training phase effectively modifies an interpretation bias, then an interaction should be observed between training valence and target valence. Participants should be faster to judge negative interpretations of ambiguous words (e.g., Terminal Cancer) as being related over benign interpretations (e.g., Terminal - Airport). The opposite pattern is predicted for benign training.

\section{Method}

\section{Participants}

Based on a review of literature that used a similar training procedure, I determined an adequate sample size to be 32 participants per training condition. Seventy-one undergraduate students (29 men, 42 women; mean age: 19.25 years) participated for course credit. All were native English speakers with normal or corrected-to-normal vision. Participants reported no current or previous diagnoses of depression. The study was approved by the Human Ethics Committee at Victoria University of Wellington. All participants gave written informed consent prior to participation.

\section{Stimuli and Apparatus}

Training and test stimuli were those developed in the pilot stages.

\section{Procedure}

Participants were randomly assigned to receive either negative or benign training. Training commenced with a centrally presented sentence (e. g., The boss wants to see you) and was followed, after a self-paced key press, by a negative or benign word fragment (e. g. , f_red [fired] or pr_moted [promoted]). 
When participants had solved the word fragment they pressed the spacebar and then typed the answer in full. Participants completed three practice trials before completing 45 training sentences. During the test phase, all participants completed the same semantic priming task to assess the presence of an interpretation bias. An ambiguous prime was presented centrally on the screen (e. g., shoot) for $250 \mathrm{~ms}$ followed by a target that was either related (e. g., gun or bamboo) or unrelated (e. g. , lonely or canoe) to the negative or benign meaning of the prime. The time between the onset of the prime and the onset of the target (stimulus onset asynchrony; SOA) was 750ms. Participants indicated whether the target was related or unrelated to the prime by pressing the 1 or 2 key on the number pad of the keyboard. Participants responded to 104 trials presented in random order; 52 targets were related to the prime (26 negative targets and 26 benign targets in each relatedness condition) and 52 were unrelated. The target remained on screen for $2000 \mathrm{~ms}$, or until the participant made a response, and was followed by an inter-trial interval of $1000 \mathrm{~ms}$. The between-subjects variable was training condition (negative or benign), and the within-subjects variables were target valence (negative or benign) and target relationship (related or unrelated).

\section{Results}

The mean time taken to correctly complete word fragments during the training phase was $2661 \mathrm{~ms}$ and the overall accuracy was $94 \%$. The time taken to complete word fragments did not vary as a function of training condition $t(64)=$ $.412, p=0.68$. Results focus on performance in the test phase as a function of training valence condition.

Accuracy and median response times for correct trials were calculated for each condition (see Table 5). 


\section{Table 5}

Mean Response Times and Accuracy of Subjects' Responses to Targets as a Function of Training for Experiment 1a (with Standard Deviations in Parentheses)

\begin{tabular}{|c|c|c|c|c|}
\hline \multirow[b]{2}{*}{ Training Valence } & \multicolumn{2}{|c|}{ Related Target Valence } & \multicolumn{2}{|c|}{ Unrelated Target Valence } \\
\hline & Negative & Benign & Negative & Benign \\
\hline & \multicolumn{4}{|c|}{ Response Times (ms) } \\
\hline Negative & $919(180)$ & $961(150)$ & $1117(268)$ & $990(322)$ \\
\hline \multirow[t]{2}{*}{ Benign } & 943(199) & $902(174)$ & $1021(240)$ & $933(230)$ \\
\hline & \multicolumn{4}{|c|}{ Accuracy } \\
\hline Negative & $0.83(0.12)$ & $0.84(0.11)$ & $0.84(0.18)$ & $0.90(0.12)$ \\
\hline Benign & $0.78(0.14)$ & $0.82(0.11)$ & $0.88(0.11)$ & $0.93(0.09)$ \\
\hline
\end{tabular}

Response times shorter than $250 \mathrm{~ms}$ were excluded from analyses as anticipatory. Although response times for unrelated trials are reported (see Table 5), the primary analyses focused on the effects of training on judgments of "related" word pairs.

Semantic priming studies using a lexical decision task often calculate a priming measure (the response time advantage for related over unrelated trials); however, such a measure is inappropriate to use with a relatedness task, because the related judgement "yes" differs in many ways from the unrelated judgement "no"2. A relatedness judgement is essentially a signal detection task, so in addition to response time and accuracy, performance was transformed into a sensitivity ( $d$ ') measure to indicate participants' ability to discriminate between related and unrelated targets. This measure was used to identify outliers with

${ }^{2}$ I will use a lexical decision task in Experiment 5 to examine priming in more depth. 
poor discrimination. A 'hit' was defined as a correct "related" response to a related target and a 'false alarm' was defined as giving an incorrect "related" response to an unrelated target. Sensitivity was calculated according to the formula:

$$
d^{\prime}=\mathrm{z} \text { (Hit Rate) - z (False Alarm Rate) }
$$

Higher scores indicate a greater ability to discriminate between related and unrelated targets. Two sensitivity scores were calculated for each participant; their $d^{\prime}$ scores to negative and benign related prime-target pairs. A criterion for participant inclusion was established so that any participant with a d' score of greater than one in at least one target condition (negative or benign) was included. This criterion led primarily to the elimination of participants who tended to call every prime-target pair related. Five participants were removed from the analysis because their $d^{\prime}$ values were less than 1.0 in at least one condition. There were 33 participants remaining in each training condition. Interpretation bias is indicated by an interaction between training and target valence such that responses are facilitated for training-congruent target valences.

\section{Response Times}

Median Response times (RTs) to related targets were analysed in a 2 (Training: negative vs. benign) x 2 (Target Valence: negative vs. benign) mixed analysis of variance (ANOVA) with Training as a between-subject variable and Target Valence as a within-subject variable. A significant Training by Target Valence interaction was observed $F(1,64)=6.79, p=.01, \eta_{p}^{2}=.09$ (see Figure 5). Paired-samples t-tests revealed that participants in the Benign Training condition showed a marginal benign bias; they were faster to judge benign associates as related to the ambiguous homographs $(M=902 \mathrm{~ms}, S D=174)$ than negative associates $(M=943 \mathrm{~ms}, S D=199), t(33)=-1.67, p=.10 d=-0.22$. In contrast, participants in the Negative Training condition showed a negative bias; they were significantly faster to judge negative associates as related to the ambiguous homographs $(M=919 \mathrm{~ms}, S D=180)$ than benign associates $(M=$ 961ms, $S D=150), t(33)=2.06, p=.04 d=.25$. This Training $\mathrm{x}$ Target Valence interaction indicates successful induction of an interpretation bias. 


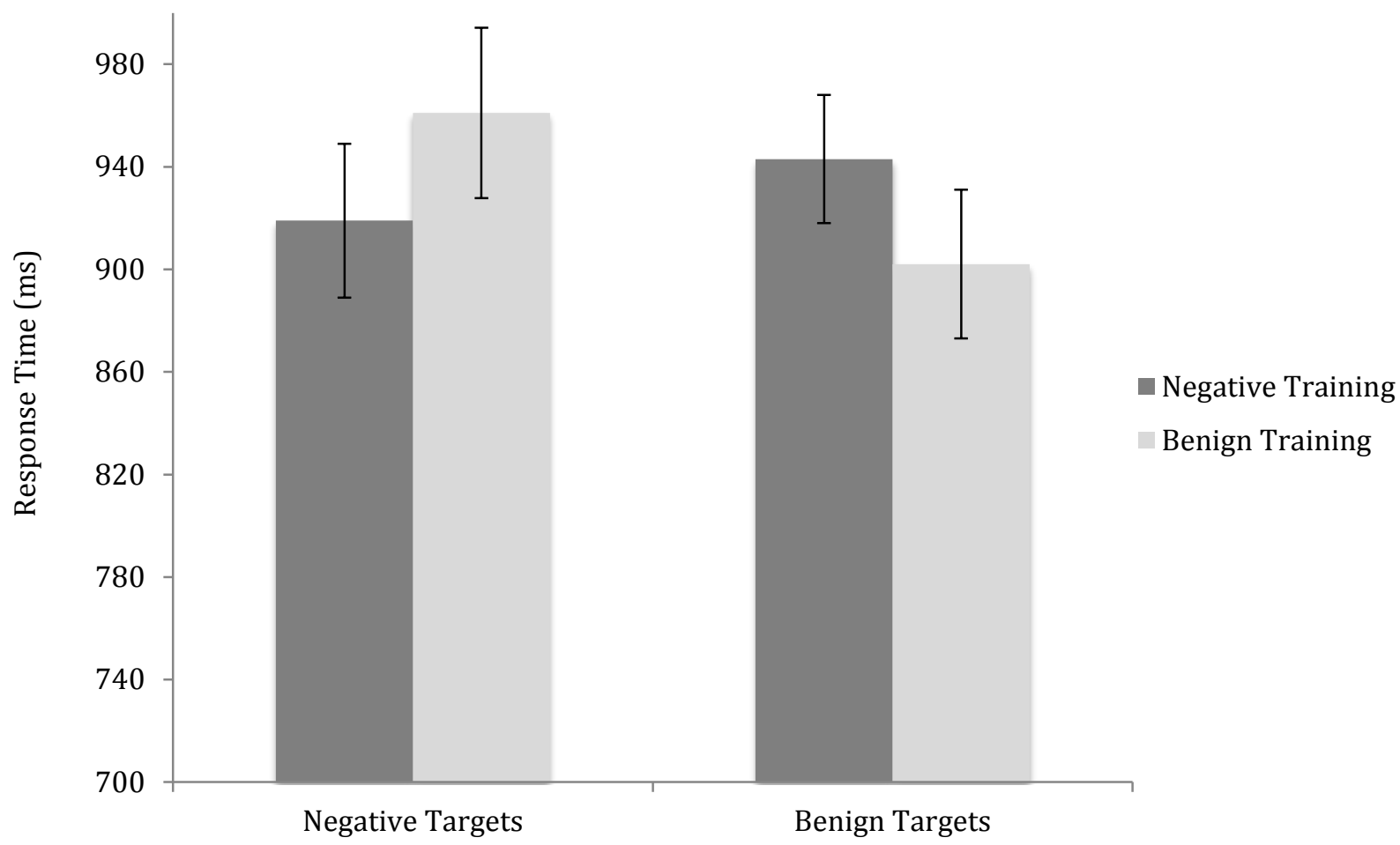

Figure 5. Response times (ms) to negative and benign targets as a function of training valence for Experiment 1a. Errors bars are Standard Errors.

\section{Accuracy}

Accuracy was calculated as the proportion of correct responses made to negative and benign related targets (i.e., Hits). Accuracy was analysed similarly to RT in a 2 (Training: negative vs. benign) x 2 (Target Valence: negative vs. benign) mixed ANOVA. The Training x Target Valence interaction was not significant, $F(1$, $64)=2.59, p=.11, \eta_{p}^{2}=.04$, however the pattern of results mirrored that in the RT analyses (See Table 5). A main effect of Target Valence approached significance, in that participants were more accurate in judging relatedness between ambiguous primes and their benign interpretations as opposed to their negative interpretations $F(1,64)=3.03, p=.08, \eta_{p}^{2}=.04$. Similar effects were observed in $d^{\prime}$. Median $d^{\prime}$ scores were analysed similarly to RT in a 2 (Training: negative vs. benign) x 2 (Target Valence: negative vs. benign) mixed ANOVA. There was a main effect of valence, $F(1,64)=19.76, p<.001, \eta_{p}^{2}=.02$, participants were better able to discriminate between related and unrelated words when the target was benign $(M=2.58, S D=.87)$ than when the target was 
negative $(M=2.2, S D=.70)$. No other significant effects were observed.

\section{Experiment 1b}

In order to determine the reliability of the effects seen in Experiment $1 \mathrm{a}$, a direct replication was run with a new group of participants.

\section{Method}

\section{Participants}

60 undergraduate students (15 men, 45 women; mean age: 21.34 years) participated for course credit. All were native English speakers with normal or corrected-to-normal vision. Participants reported no current or previous diagnoses of depression. The study was approved by the Human Ethics Committee at Victoria University of Wellington. All participants gave written informed consent prior to participation.

\section{Stimuli and Apparatus}

Stimuli and Apparatus were identical to that of Experiment 1.

\section{Procedure}

Participants were run in groups of four, at individual testing carols. The procedure was otherwise identical to that of Experiment 1.

\section{Results}

The mean time taken to correctly complete word fragments during the training phase was $2036 \mathrm{~ms}$ and the overall accuracy was $94 \%$. Response times to complete word fragments did not differ by training condition $t(52)=1.46, p=$ 0.15. Accuracy and mean response times for correct trials were calculated for each condition (see Table 6). Six participants were removed from the analysis because their $d^{\prime}$ values were less than 1.0 in at least one condition. There were 27 participants remaining in each training condition.

\section{Response Times}

Response times (RTs) to related targets were analysed in a 2 (Training: negative vs. benign) x 2 (Target Valence: negative vs. benign) mixed analysis of variance (ANOVA) with Training as a between-subject variable and Target Valence as a within-subject variable. A significant Training by Target Valence interaction was observed $F(1,52)=5.34, p=.02, \eta_{p}^{2}=.09$ (see Figure 6). Paired- 
samples t-tests revealed that participants in the Benign Training condition were significantly faster to judge benign associates of ambiguous primes as related than negative associates, $t(26)=2.57, p=.02, d=.19$. Participants in the Negative Training condition showed a trend in the opposite direction; they were marginally faster to judge negative associates as related than benign associates $t(26)=-1.16, p=.20,=.21$. This Training $\mathrm{x}$ Target Valence interaction is very similar to that seen in Experiment 1, and indicates successful induction of an interpretation bias

\section{Accuracy}

Accuracy was calculated as the proportion of correct responses made to negative and benign related targets. Accuracy was analysed similarly to RT in a 2 (Training: negative vs. benign) x 2 (Target Valence: negative vs. benign) mixed ANOVA. No significant effects were observed.

\section{Table 6}

Mean Response Times and Accuracy of Subjects' Responses to Targets as a Function of Training for Experiment $1 b$ (with Standard Deviations in Parentheses)

\begin{tabular}{|c|c|c|c|c|}
\hline \multirow[b]{2}{*}{ Training Valence } & \multicolumn{2}{|c|}{ Related Target Valence } & \multicolumn{2}{|c|}{ Unrelated Target Valence } \\
\hline & Negative & Benign & Negative & Benign \\
\hline & \multicolumn{4}{|c|}{ Response Times (ms) } \\
\hline Negative & $848(115)$ & $872(130)$ & $959(207)$ & $891(173)$ \\
\hline \multirow[t]{2}{*}{ Benign } & $841(115)$ & 809 (99) & $904(164)$ & $891(170)$ \\
\hline & \multicolumn{4}{|c|}{ Accuracy } \\
\hline Negative & $0.75(0.12)$ & $0.75(0.12)$ & $0.86(0.12)$ & $0.92(0.08)$ \\
\hline Benign & $0.78(0.12)$ & $0.78(0.11)$ & $0.86(0.18)$ & $0.92(0.16)$ \\
\hline
\end{tabular}


Median $d^{\prime}$ scores were analysed similarly to RT in a 2 (Training: negative vs. benign) x 2 (Target Valence: negative vs. benign) mixed ANOVA. There was a main effect of valence, $F(1,52)=11.61, p<.001, \eta_{p}^{2}=.18$, participants were better able to discriminate between related and unrelated words when that target was benign $(M=2.46, S D=.72)$ than when the target was negative $(M=$ $2.07, S D=.66)$. No other significant effects were observed.

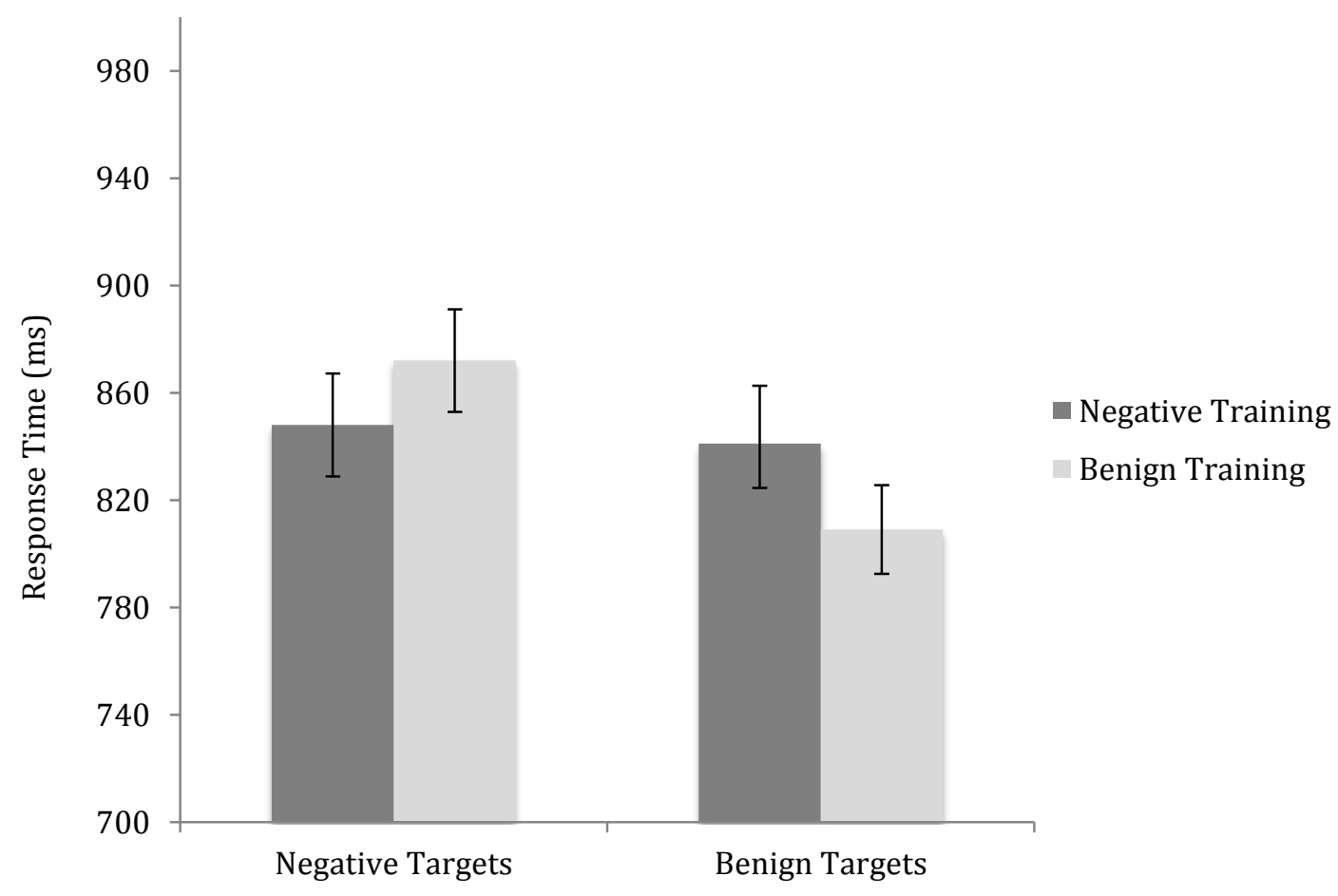

Figure 6. Response times (ms) to negative and benign targets as a function of training valence for Experiment $1 b$.

\section{General Discussion}

The results of Experiment $1 \mathrm{a}$ reveal the successful induction of an interpretive bias following a novel scenario-based training phase. After benign training, participants were faster to respond to targets related to benign meanings of ambiguous words relative to negative meanings. However, after negative training, participants were instead faster to make relatedness decisions to negative associates. The results of Experiment $1 \mathrm{~b}$ replicate the findings of Experiment 1a, bolstering the evidence that the novel CBM-I paradigm successfully modifies interpretative bias. 
It is unsurprising that the interaction between training and target valence was not significant in the accuracy data. In many priming tasks accuracy is typically quite high and as a result, the speed at which participants make responses is a better measure of the availability of meanings instead. Despite this, a clear trend towards an interaction between training valence and target valence was evident in Experiment $1 \mathrm{a}$, and the data show no indication of a speed-accuracy trade-off.

The training procedure differed slightly from the traditional scenariobased training paradigm, in which the word fragment is typically the last word of the sentence or scenario and so ultimately constrains the emotional interpretation. In this paradigm, the sentence itself remained ambiguous, but the fragment was consistently related to either its negative or benign interpretation. Despite procedural differences, training produced a typical interpretation bias in both experiments, as indicated by the Training $x$ Target Valence interaction in response times. Moreover, the effect size for the critical interaction $\left(\eta_{p}^{2}=.09\right.$ in both Experiment 1a and 1b) was similar to that reported in other studies that use semantic priming tasks to assess interpretation (e.g., Hoppitt et al., 2010a, $\eta_{p}^{2}=$ $.12)$.

By using an online priming task I was able to assess the interpretation of emotional ambiguity almost immediately (750 ms) after the presentation of an ambiguous word. This online task contrasts with delayed tasks such as a recognition memory task that can reflect biases introduced by reinterpretation in memory or decision-making processes. The systematic piloting and construction of balanced stimuli lists also makes it unlikely that these biases are due to differences in the orthographic properties of the stimuli. The results of Experiment $1 \mathrm{a}$ and $1 \mathrm{~b}$ give considerable weight to the argument that the training phase directly affects participants' later interpretation of emotional ambiguity in the test phase.

Another consideration is the overall benign bias seen in related target RT performance. One explanation for the benign bias could be that judging whether two benign words are related is simply an easier decision than judging the relationship between two negative words. Negative valence may reflect a stronger (or more coherent) semantic category than benign valence, meaning 
that all negative words seem more likely to be related due to their shared emotional category. With this emotional connection in mind it may be more difficult to reject Punch-Coward as a related pair than Desert-Bacon. Without a no-training control condition it is impossible to determine whether participants in the benign training condition show facilitation for benign targets relative to participants who have not gone through any training. A no-training control condition is rarely used in CBM-I experiments (Hirsch, Hayes \& Mathews, 2009; Mobini et al., 2014) and so a conservative conclusion is that, relative to benign training, negative training causes a shift in meaning selection. An alternative explanation for the benign bias is that it may reflect a failure to properly balance stimuli for meaning dominance (despite all efforts during piloting), but it may also reflect a positivity bias.

Finally, the findings of Experiment $1 \mathrm{a}$ and Experiment $1 \mathrm{~b}$ bolster the suggestion that an interpretive bias has been modified following the novel scenario-based CBM-I paradigm. 


\section{Chapter 3: Investigating the Time-Course of Interpretive Bias Modification}

The results of Chapter 2 suggest that the novel CBM-I paradigm used in Experiments $1 \mathrm{a}$ and $1 \mathrm{~b}$ shifted interpretive biases in healthy participants. $\mathrm{A}$ further question that now arises concerns the emergence of such biases. How do interpretive biases unfold over time? At what lexical stages, (i.e., activation or selection) can they be observed? According to the widely accepted two-stage models of lexical ambiguity resolution (e.g., The Reordered Access Model: Duffy et al. 1988 and The Independent Activation Model; Twilley \& Dixon, 2000), biases in meaning availability could appear as early as the stage at which each meaning is activated. Alternatively, differences may not emerge until a later stage when meaning selection occurs.

The two mechanistic accounts of interpretive bias make different predictions about how these differences may arise following training. According to an Emotional Priming Account, training serves as a global emotional context, which influences the availability of all emotionally-congruent meanings. Emotional priming accounts therefore predict that training effects could appear either at an early meaning activation stage or a later selection stage. Alternatively, according to an Ambiguity Resolution Account the training phase fosters the acquisition of a learned production rule, which affects the later selection of one meaning of an ambiguous word. Therefore, training should only have an effect at the later selection stage, and not at the earlier activation stage.

One way to observe changes in lexical processing over time is to manipulate the Stimulus Onset Asynchrony (SOA), the time between the presentation of a prime and a target. A participant's response reflects the influence of the prime on the processing of the target at the moment it appears. At short SOAs (i.e., under $300 \mathrm{~ms}$ ), all possible meanings of the prime will have been activated, but not selected, when the target appears. At long SOAs, the meaning of the prime will have been both activated and selected. A number of studies using variable SOAs have shed light on the changing pattern of activation in semantic memory at different intervals (e.g., Swinney, 1979, Tanenhaus, Leiman \& Seidenberg, 1979, Simpson \& Burgess, 1985). Importantly, context can have different effects at different stages of meaning selection.

Early lexical access is believed to be fast and automatic and later strategic 
processes are thought to be driven by contextual effects (e.g., Posner \& Snyder, 1975; Shiffrin \& Schneider, 1977). Early automatic effects of semantic priming have been well-documented. For example, Neely (1977) examined the effects of expectancy and semantic relatedness in a lexical decision task with variable SOAs. Participants were told to expect targets related to "building" concepts (e.g., window, door) to always follow primes related to body concepts (e.g., legwindow). However, sometimes prime-target pairs, which were not expected but were semantically related appeared (e.g., door-window). Prior to $450 \mathrm{~ms}$, only lexical decisions to targets that were semantically related to the primes were facilitated. However, after 2 seconds, only lexical decisions to expected relationships were facilitated and not those to semantically associated targets. It therefore appears that intentional processing of the prime-target relationship does not emerge in the early stages of lexical access. The next question is then, how does emotion fit with these findings? Does the emotional context established by the training phase act similarly to established semantic relationships and drive the automatic activation of meanings, similar to the affective priming effect? Or do the effects of the training phase only emerge later, to guide strategic selection of an appropriate meaning?

Effects of emotion on unambiguous word recognition have been observed at short SOAs in a number of priming studies (Spruyt, Hermans, De Houwer, Vandromme \& Eelen, 2007; Hermans et al., 2001). For example, Klauer, Robnagel and Musch (1997) presented participants with prime-target pairs consisting of negative or positive word combinations and participants judged if the second word was positive or negative. At a short SOA (100ms) participants showed facilitated performance for congruent relative to incongruently valenced word pairs. This suggests the existence of a very early affective priming effect, resulting from boosted activation of similarly valenced words. Prior exposure to an emotional context may therefore speed activation to emotionally-congruent meanings of an emotionally ambiguous prime. This activation may only last long enough to affect lexical access but not selection, which occurs after 500ms. No priming was observed at the longer SOA's, which the authors took to mean that evaluative priming effects are short-lived and decay quickly when alternative strategic processes are not recruited. 
Broader (non-emotional) contextual effects in priming studies are not observed until later processing stages. For example studies using ambiguous primes (e.g., bugs) embedded in a sentence, have found that upon initial presentation of the prime, priming occurs for both meanings (e.g., ant and spy) however, after a delay priming is only observed for the contextually appropriate meaning (e.g., Swinney, 1979). This is in line with the theorized timing of strategic processes that integrate the word into the broader sentence context following activation of meaning candidates. In regards to emotional context, Richard and French (1992) found that lexical decisions to threatening interpretations of ambiguous words were facilitated for high anxious participants after $750 \mathrm{~ms}$ but not at a $500 \mathrm{~ms}$ SOA. This suggests that in anxious populations, mood congruent biases for meanings of ambiguous words appear around the same time that sentential context is integrated into the selection of meanings (Swinney, 1979). Other studies that have looked at the emotional priming effect at long SOAs suggest that these slower-acting context effects reflect "controlled" strategic processes (e.g., de Groot, 1984; Neely, 1991; Seidenberg, 1985). The effects of emotional context on early and late processing stages can be described as reflecting local and global context, respectively. Local context effects seen in affective priming tasks result in fast and early activation of congruent meanings, whilst global context reflects the slower effect of contextual integration with a broader context or discourse at a later selection stage.

Experiment $1 \mathrm{a}$ and $1 \mathrm{~b}$ used a relatively long SOA (750 ms), which allowed time for both activation and selection of the meaning of the prime to occur before the onset of the target (e. g., Neely, 1977; Swinney, 1979). By presenting the target earlier (i.e., by using a shorter SOA) it is possible to tap into the earlier activation stage of lexical processing, before a participant has selected a particular meaning of the ambiguous prime word. If training biases the activation levels of valenced meanings of an ambiguous prime, then training effects should be observed at both the short and long SOA. However, if training only biases the selection of meanings, then training effects should only be apparent in the long SOA condition (i.e., Experiment $1 \mathrm{a}$ and $1 \mathrm{~b}$ ). The idea of biased selection is in line with models of ambiguity resolution (e.g., Duffy et al., 1988, Twilley \& Dixon, 2000), which state that meaning activation cannot be constrained in such a way 
that only one meaning becomes activated. However, the amount of activation each meaning receives can be modified by contextual and frequency variables (e.g., Oden \& Spira, 1983; Simpson \& Burgess, 1985). Importantly, according to an Ambiguity Resolution Account, interpretive biases should only emerge at a long SOA due to the induction of a production rule that requires the recruitment of strategic processing, and should therefore not be observed at a shorter SOA However, according to an Emotional Priming Account, which states that an interpretive bias reflects a broad activation of training-congruent emotional networks, an interpretive bias might appear at either a short or a long SOA.

\section{Experiment 2}

\section{Participants}

74 undergraduate students (26 men, 48 women; mean age: 19.68 years) participated for course credit. All were native English speakers with normal or corrected-to-normal vision. Participants reported no current or previous diagnoses of depression. The study was approved by the Human Ethics Committee, Victoria University of Wellington. All participants gave written informed consent prior to participation.

\section{Stimuli and Apparatus}

Stimuli and Apparatus were identical to that of Experiment 1.

\section{Procedure}

The training phase was identical to that of Experiment 1. In the test phase, the time between the presentation of the prime and the target (SOA) was shortened to $250 \mathrm{~ms}$. Participants were run in groups of four.

\section{Results}

The mean time taken to correctly complete word fragments during the training phase was $2136 \mathrm{~ms}$ and the overall accuracy was 95\%. Time taken to complete word fragments did not differ by training condition $t(67)=.658, \mathrm{p}=$ 0.51. Results focus on performance in the test phase as a function of training. Accuracy and mean response times for correct trials were calculated for each condition (see Table 7). Five participants were removed from the analysis because their $d^{\prime}$ values were less than 1.0 in at least one condition. There were 35 participants in the Negative Training Condition and 34 in the Benign Training 
Condition.

Table 7

Mean Response Times (ms) and Accuracy of Subjects' Responses to Targets as a Function of Training for Experiment 2(with Standard Deviations in Parentheses)

\begin{tabular}{|c|c|c|c|c|}
\hline \multirow[b]{2}{*}{ Training Valence } & \multicolumn{2}{|c|}{ Related Target Valence } & \multicolumn{2}{|c|}{ Unrelated Target Valence } \\
\hline & Negative & Benign & Negative & Benign \\
\hline & \multicolumn{4}{|c|}{ Response Times (ms) } \\
\hline Negative & $839(150)$ & $832(128)$ & $937(168)$ & $859(152)$ \\
\hline \multirow[t]{2}{*}{ Benign } & $843(152)$ & $799(133)$ & $931(184)$ & $889(138)$ \\
\hline & \multicolumn{4}{|c|}{ Accuracy } \\
\hline Negative & $0.76(0.11)$ & $0.78(0.10)$ & $0.88(0.10)$ & $0.91(0.09)$ \\
\hline Benign & $0.75(0.12)$ & $0.82(0.11)$ & $0.90(0.09)$ & $0.94(0.05)$ \\
\hline
\end{tabular}

\section{Response Times}

Response times (RTs) to related targets were analysed in a 2 (Training: negative vs. benign) x 2 (Target Valence: negative vs. benign) mixed analysis of variance (ANOVA) with Training as a between-subject variable and Target Valence as a within-subject variable. The interaction did not approach significance, $F(1,67)=.04, p=.84, \eta_{p}^{2}=.01$, indicating that participants did not differ in their speed of relatedness judgments as a function of training. This finding indicates a failure to modify interpretive biases in participants. A main effect of valence was found whereby participants were faster to identify benign associates of ambiguous primes $(\mathrm{M}=816 \mathrm{~ms}, \mathrm{SD}=132)$ as related than negative associates $(\mathrm{M}=841 \mathrm{~ms}, \mathrm{SD}=150), F(1,67)=33.05, p<.001, \eta_{p}^{2}=.33$. No other significant effects were observed.

\section{Accuracy}


Accuracy was calculated as the proportion of correct responses made to negative and benign related targets (i.e., hits). Accuracy was analysed similarly to RT in a 2 (Training: negative vs. benign) x 2 (Target Valence: negative vs. benign) mixed ANOVA. A main effect of valence was found whereby participants were more accurate at judging the relationship between benign associates of ambiguous primes $(\mathrm{M}=.80, \mathrm{SD}=.10)$ than negative associates $(\mathrm{M}=.76, \mathrm{SD}=.11)$, $F(1,67)=7.40, p=.008, \eta_{p}^{2}=.10$. Median $d^{\prime}$ 'scores were analysed similarly to RT in a 2 (Training: negative vs. benign) x 2 (Target Valence: negative vs. benign) mixed ANOVA. There was a main effect of valence, $F(1,67)=23.7, p<.001, \eta_{p}^{2}=$ .26 , participants were better able to discriminate between related and unrelated words when the target was benign $(M=2.54, S D=.62)$ than when the target was negative $(M=2.16, S D=.57)$. No other significant effects were observed.

\section{General Discussion}

The results of Experiment 2 reflect a failure to find any modification of interpretation bias following training when the SOA in the test phase was shortened to $250 \mathrm{~ms}$. During the test phase, participants were neither faster nor more accurate to respond to negative or benign targets as a function of training. These findings suggest that the interpretive biases observed in Experiments $1 \mathrm{a}$ and $1 \mathrm{~b}$ emerge by $750 \mathrm{~ms}$ but not after $250 \mathrm{~ms}$. The training appears to act as a contextual cue that guides the selection of a training-congruent meaning

There was a general advantage for benign over negative meanings in both accuracy and RT. The bias towards benign meanings at the short SOA in this experiment is similar to that seen in the benign training condition at the long SOA (59ms advantage in $1 \mathrm{a}, 63 \mathrm{~ms}$ advantage in $1 \mathrm{~b}$ and $25 \mathrm{~ms}$ here). The stable benign bias seen across experiments may either reflect a stable benign bias characteristic of healthy populations or it may suggest that the benign relationship decision is simply an easier one relative to negative relationship decisions.

Overall, the results suggest that the effects of training only emerge at a later selection stage following the activation of multiple meaning candidates. The non-significant interaction between training and target valence at a short SOA this argues against an early affective priming effect. However, the question 
remains whether interpretation change at the meaning selection stage arises through emotional priming or through an implicit production rule, or both. 


\section{Chapter 4: The role of ambiguity resolution in training}

Experiments in Chapter 2 established that the scenario-based training paradigm altered interpretive biases in the test phase, and findings from Chapter 3 indicated that training altered the later meaning selection, and not the earlier activation stage of processing. But how does training affect meaning selection? Both Emotional Priming and Ambiguity Resolution Accounts can explain the effects of training on meaning selection, but they do so through different mechanisms. According to an Emotional Priming Account, repeatedly accessing valenced information during training facilitates later selection of similarly valenced information. Late-acting emotional priming effects are proposed to occur via priming throughout an attractor network (Becker et al.,, 1997).

Alternatively, according to an Ambiguity Resolution Account, the training phase provides procedural context whereby participants learn a given response in the presence of emotional ambiguity. A participant learns an implicit production rule over the course of the experiment; to select one meaning over another in a possible 'IF in the presence of emotional ambiguity THEN select a negative interpretation" manner. One possible explanation for the acquisition and application of procedural rules can be surmised from the implicit knowledge literature (e.g., Lewiki, Hill \& Bizot, 1988). This approach is developed from evidence that the cognitive system can implicitly detect complex relationships between different features of stimuli. After acquisition, the procedural rule can then be applied and generalized to encounters with novel stimuli. Similar procedural rules have been successfully induced in participants in other contexts (Hill, Lewicki, Czyzewska \& Boss, 1989). In an initial learning phase participants saw six short videos of men and women completing mundane activities, dubbed over with a spoken negative or benign internal monologue. The negative monologues described a person's hidden sadness or anxiety while the benign monologues detailed routines of college life. Gender was manipulated so that half of participants heard the woman's monologue, and half heard the man's monologue. Afterwards, participants were presented with a list of personality and mood dimensions (e.g., Optimistic-Pessimistic, Sad-Happy) and were asked to rate male and female acquaintances on each characteristic. Participants in the sad male monologue condition rated their male acquaintances as being more sad 
than their female acquaintances. Similar learning-congruency effects were observed in the sad female condition. Participants learned associations between two features (gender and personality characteristics) and generalized this rule in order to interpret ambiguous information ${ }^{3}$.

Each account predicts that different features of the training paradigm are critical to success. According to an Emotional Priming Account the only necessary requirement of a training phase is that the participant repeatedly activates or accesses valenced information. However, according to an Ambiguity Resolution Account, activation of an emotional category is insufficient to modify a bias; the participant must instead learn a production rule by repeatedly being presented with emotionally ambiguous stimuli and forced (or at least encouraged) to interpret it a particular way. One way to compare these two accounts is to modify the requirement for ambiguity resolution in the training phase. If an interpretive bias emerges through repeated exposure to, and generation of, emotional valence, then biases should be observed regardless of whether a participant is required to resolve ambiguity or not during training. Alternatively, if interpretive biases emerge due to a learned production rule then biases should only emerge when participants are required to interpret emotional ambiguity in the training phase. If interpretive biases are modified by both mechanisms then removing the need for emotional ambiguity resolution should attenuate, but not eliminate the effects of training.

The manipulation of ambiguity in the training phase was used by Clarke and colleagues (2014), who used a modified version of Mathews and Mackintosh's delayed test paradigm. The training phase had two conditions. In the Ambiguity present condition, participants heard scenarios that were emotionally ambiguous until the final word(s) was presented, which disambiguated the scenario in a negative or a benign manner e.g.,

You are skiing down a slalom slope at high speed. You fall and hear a crack. You realise that you have broken a bone/ski

\footnotetext{
${ }^{3}$ In this study, as the personality traits were considered to be internal and therefore hidden, the determintation of an acquaintance's inner sadness or happiness can be considered a task in resolving ambiguity.
} 
In the Ambiguity absent condition, participants heard similar sentences that did not require ambiguity resolution, that is, the sentences they heard were explicitly negative or benign e.g.,

You realise that you have broken your bone/ski when you fall and hear a crack. You had been skiing down a slalom slope at high speed

In the Ambiguity Present condition presumably participants start to interpret the ambiguous sentence themselves (including the ambiguous word "crack"), but a consistently negative or benign final word(s) resolves the ambiguity for them. However, in the Ambiguity Absent condition, the valence of the sentence was established up front, meaning there was no need for interpretation by the participant.

Following this initial training, all participants heard ten critical sentences that remained ambiguous e.g.,

You are trying out some new recipes you found and begin preparing a dish to serve your family that night when your partner comes in and makes a comment about the smell

In the following test phase, participants saw possible negative and benign interpretations of the critical sentences and were asked to rate them in terms of their similarity to their original interpretations of the sentences (i.e., a delayed recognition test). Consistent with an ambiguity resolution account, only participants in the ambiguity present condition rated critical sentences in a training-congruent manner. These findings suggest that both activation and selection during meaning sense disambiguation are required in the training phase in order for interpretive biases to be observed at test. Note however that the experimenters used a delayed task at test, and so it may be that training effects arose during memory or decision stages, and might not indicate effects of training on interpretation per se.

However, evidence in support of an Emotional Priming Account has also been observed, albeit on a methodologically different task. Some researchers 
within the CBM-I literature differentiate between active tasks - those which require the active interpretation of ambiguity - and passive tasks which do not ${ }^{4}$. For example, in Grey and Matthew's (2000) third experiment, participants completed a homograph relatedness task during the training phase and a lexical decision task during the test phase. During training, participants saw unambiguous negative or benign primes followed by ambiguous related targets (e.g., Water-Drop or Break-Drop). Note that this arrangement is the reverse of the usual training procedure, in which the ambiguous word is presented before the target. It was argued that this task was a passive one because the ambiguous target was preceded by unambiguous primes and there was therefore no need for participants to activate multiple possible interpretations of the target. During the test phase, participants saw homograph primes followed by negative and benign related targets and judged whether the target was a word or a nonword (i.e., a lexical decision task). In line with an Emotional Priming Account participants showed interpretive biases similar to those seen with traditional active training tasks such as word fragment completion training.

The authors concluded that the active interpretation of ambiguity was not necessary to shift biases at test. However, it is possible here to argue that the simple presence of ambiguity in the training task (even in the second word of the pair) meant that participants spontaneously activated multiple meanings of an ambiguous word. Despite the reversal of the traditional training paradigm all meanings of an ambiguous word are automatically activated upon onset. Therefore, the target word may not have necessarily constrained activation of a single meaning from an ambiguous word as proposed by the authors.

More compelling evidence in support of emotional priming comes from a similar study in which Hoppitt et al (2010a) conducted a fragment completion training task that was completely unambiguous. In the passive condition participants in the negative training condition saw unambiguous negative words (e.g., cancer) followed by related word targets (e.g., tumour) and participants in the benign condition saw unambiguous benign words (e.g., height) followed by related word fragments (e.g., expansion). Participants in the active condition saw

\footnotetext{
${ }^{4}$ Clarke et al.'s (2014) two conditions would both be considered passive, since the words are provided by the experimenter
} 
ambiguous primes (e.g., growth) followed by related or negative word fragments (e.g., height or expansion). The critical difference was that in passive training, the prime was unambiguous and so its meaning activation facilitated completion of the fragment. However, in active training, the homograph prime was only informative if its training-congruent meaning was activated and selected. Following training, interpretive biases were tested with a primed lexical decision task. Regardless of active or passive training condition, participants exhibited training congruent interpretive biases. However, this experiment included considerable procedural overlap between training and test, making interpretation of the training effect less clear.

The aim of the two experiments described in this chapter was to distinguish between Emotional Priming and Ambiguity Resolution accounts, by determining the role of ambiguity resolution during training in biasing the online interpretation of emotional meaning. In a typical scenario-based CBM-I training, participants read semantically- and affectively-rich sentences, they resolve emotional ambiguity, and they generate valenced words to complete the fragments. They therefore generate and select emotional meanings at a number of levels and there are therefore a number of features of the training task that could affect the later interpretation of emotional ambiguity. In Experiment 3 the association between the scenarios and the word fragments in the training phase was removed entirely in order to determine whether the generation of emotionally-valenced words alone (through the fragment completion task) was sufficient to modify biases at test. If, in line with an Emotional Priming account, the interpretation of ambiguity can be modified by the repeated generation of valenced words, then similar biases to those seen in Experiment 1 are predicted. However, if this repeated access to valenced information is not sufficient, then no Training $\mathrm{x}$ Target interaction is predicted. In Experiment 4, training sentences were rewritten so that they were no longer ambiguous; instead they now reflected unambiguous negative and benign statements related to the word fragments used in Experiment 1. This manipulation was used to assess whether repeatedly reading semantically-rich and explicitly-valenced (but not ambiguous) sentences was sufficient to shift interpretive biases, or whether the resolution of ambiguity, as in Experiment 1 is a critical determiner of bias. 


\section{Experiment 3}

The aim of Experiment 3 was to determine whether the completion of valenced word fragments during training was itself sufficient to alter an interpretation bias in line with an Emotional Priming Account. I used the same negative (e.g., $\left.a_{-} t a_{-} k[a t t a c k]\right)$ and benign (e. g. , pl_y [play]) word fragments that were used in Experiment 1; however the accompanying sentences were rewritten so that they were unambiguous, unemotional, and unrelated to the original fragments (e.g., Your sister is now working in I. T. ). Essentially the sentences acted as 'fillers' so that the structure of the training paradigm in Experiment 3 closely paralleled that in Experiments 1 and 2, but the only source of emotion was the word fragment itself. The test phase remained identical to that in Experiment 1.

\section{Method}

\section{Participants}

Seventy-four undergraduate students (20 men, 54 women; mean age: 21 . 4 years) participated for course credit. All were native English speakers with normal or corrected-to-normal vision. Participants also reported no current or previous diagnoses of depression. They were randomly assigned to receive either negative or benign training. The study was approved by the Human Ethics Committee, Victoria University of Wellington. All participants gave written informed consent prior to participation.

\section{Stimuli and Apparatus}

The training sentences were rewritten so that they were no longer related to the negative and benign word fragments. The sentences remained selfreferential but were neutral in valence. As the training sentences were no longer related to the fragments, and therefore did not facilitate fragment completion, some fragments were slightly altered after piloting by removing different letters in order to equate them for difficulty. None of the words used for the fragments changed.

\section{Procedure}

The procedure and design were identical to that of Experiment 1 . Participants were run individually. 


\section{Results and Discussion}

Accuracy and mean RTs were calculated as in Experiment 1, and are reported in Table 8. The mean time taken to correctly complete word fragments in the training phase was $2547 \mathrm{~ms}$ and the overall accuracy was 96.3\%. Four participants were excluded from analysis based on the previous accuracy exclusion criteria set for Experiment $1\left(d^{\prime}<1.0\right.$ in at least one condition). Analyses were conducted on the remaining 70 participants, 34 in the negative training condition, and 36 in the benign training condition.

\section{Table 8}

Mean Response Times (ms) and Accuracy of Subjects' Responses to Targets as a Function of Training for Experiment 3 (with Standard Deviations in Parentheses)

\begin{tabular}{|c|c|c|c|c|}
\hline \multirow[b]{2}{*}{ Training Valence } & \multicolumn{2}{|c|}{ Related Target Valence } & \multicolumn{2}{|c|}{ Unrelated Target Valence } \\
\hline & Negative & Benign & Negative & Benign \\
\hline & \multicolumn{4}{|c|}{ Response Times (ms) } \\
\hline Negative & 947 (204) & $911(150)$ & 1057 (297) & $996(261)$ \\
\hline \multirow[t]{2}{*}{ Benign } & $904(168)$ & $879(152)$ & 1010 (269) & $968(301)$ \\
\hline & \multicolumn{4}{|c|}{ Accuracy } \\
\hline Negative & $0.78(0.10)$ & $0.81(0.09)$ & $0.90(0.10)$ & $0.95(0.05)$ \\
\hline Benign & $0.79(0.11)$ & $0.80(0.08)$ & $0.90(0.08)$ & $0.95(0.06)$ \\
\hline
\end{tabular}

\section{Response Times}

RTs to related targets were analysed in a 2 (Training: negative vs. benign) x 2 (Target Valence: negative vs. benign) mixed ANOVA with Training as a between-subject variable and Valence as a within-subject variable. Importantly the interaction did not approach significance, $F(1,68)=.12, p=.74, \eta_{p}^{2}=.01$, 
indicating that the completion of valenced fragments did not alter participants' interpretation biases. A main effect of valence was found whereby participants were faster to identify benign associates of ambiguous primes as related than negative associates, $F(1,68)=3.86, p=.05, \eta_{p}^{2}=.05$. No other significant effects were observed.

\section{Accuracy}

Accuracy on related trials was analysed in a 2 (Training: negative vs. benign) x 2 (Target Valence: negative vs. benign) mixed ANOVA. No significant effects were observed. Median $d^{\prime}$ scores were analysed similarly to RT in a 2 (Training: negative vs. benign) x 2 (Target Valence: negative vs. benign) mixed ANOVA. There was a main effect of valence, $F(1,68)=45.46, p<.001, \eta_{p}^{2}=.394$. Participants were better able to discriminate between related and unrelated word pairs when the target was benign $(M=2.7, S D=.61)$ than when the target was negative $(M=2.28, S D=.57)$. No other significant effects were observed.

The results of Experiment 3 argue against a simple Emotional Priming Account of modified interpretive biases. Repeatedly accessing negative information via word fragment completion was not sufficient to induce an interpretation bias. Again, an advantage for benign interpretation of emotionally ambiguous words was observed that did not interact with training. However, it is not yet clear whether the Ambiguity Resolution Account might still be supported. It is possible that in Experiment 1, reading semantically rich, descriptive scenarios in combination with the word fragment completion task enhanced activation of a valenced context In order to test this account a further study used unambiguous but emotionally-valenced senteneces, to asses whether the resolution of ambiguity or the exposure to emotional scenarios is a critical factor involved in the induction of an interpretation bias.

\section{Experiment 4}

The aim of Experiment 4 was to further examine the role of ambiguity resolution in inducing an interpretation bias. If the repeated resolution of emotional ambiguity is a requirement of the training phase, then valenced (but unambiguous) scenarios should not modify interpretive biases in a similar way to those observed in Experiment 1. The sentences from Experiment 1 were 
reconstructed to become unambiguously negative and benign statements. For example, the ambiguous sentence The dog is running toward you was altered to read either The angry dog is running towards you or The friendly dog is running towards you (depending on the training condition). Thus, the sentences kept the meanings of the original two possible interpretations, but those meanings were presented to participants unambiguously. Participants then completed a related word fragment. This manipulation produced meanings that were possibly more clearly emotional than the ambiguous sentences. Thus, according to an Emotional Priming Account, it is possible that these sentences could produce an even stronger training effect than seen in Experiment 1. However, if, as predicted by an Ambiguity Resolution Account, the disambiguation of emotional ambiguity is a necessary feature of the training phase, then removing the requirement for participants to interpret emotional ambiguity during training should eliminate the training effects seen at test.

\section{Method}

\section{Participants}

Seventy-four undergraduate students (all women; mean age: 18.60 years) participated for course credit. All had normal or corrected-to-normal vision and English was their native language. Participants also reported no current or previous diagnoses of depression. They were randomly assigned to receive either negative or benign training. The study was approved by the Human Ethics Committee, Victoria University of Wellington. All participants gave written informed consent prior to participation.

\section{Stimuli and Apparatus}

An unambiguous negative sentence and an unambiguous benign sentence were constructed from each original ambiguous sentence used in Experiment 1. For example, the ambiguous scenario, You are going to a party where you don't know anyone (Word Fragment: scared/excited) became either You are not looking forward to the party (Word Fragment: scared) or You are looking forward to the party (Word Fragment: excited), depending on the training condition. Otherwise all materials were the same as those in Experiment 1 (See Appendix B). 


\section{Procedure}

The procedure was identical to that of Experiment 1. Participants were run individually.

\section{Results and Discussion}

Accuracy and mean RTs were calculated as in Experiment 1, and are reported in Table 9. The mean time taken to correctly complete word fragments in the training phase was $2484 \mathrm{~ms}$ and the mean overall accuracy was $95 \%$. Three participants were excluded from analysis based on the accuracy exclusion criteria as in for Experiment $1\left(d^{\prime}<1.0\right.$ in at least one condition). Analyses were conducted on data from the remaining 71 participants; 34 in the negative training condition and 37 in the benign training condition.

\section{Table 9}

Mean Response Times (ms) and Accuracy of Subjects' Responses to Targets as a Function of Training for Experiment 4 (with Standard Deviations in Parentheses)

\begin{tabular}{|c|c|c|c|c|}
\hline \multirow[b]{2}{*}{ Training Valence } & \multicolumn{2}{|c|}{ Related Target Valence } & \multicolumn{2}{|c|}{ Unrelated Target Valence } \\
\hline & Negative & Benign & Negative & Benign \\
\hline & \multicolumn{4}{|c|}{ Response Times (ms) } \\
\hline Negative & $940(196)$ & $932(146)$ & 1101 (249) & $1069(262)$ \\
\hline \multirow[t]{2}{*}{ Benign } & $912(166)$ & $889(136)$ & 1057 (161) & $1002(194)$ \\
\hline & \multicolumn{4}{|c|}{ Accuracy } \\
\hline Negative & $0.79(0.11)$ & $0.78(0.09)$ & $0.88(0.11)$ & $0.96(0.03)$ \\
\hline Benign & $0.80(0.10)$ & $0.83(0.10)$ & $0.87(0.12)$ & $0.93(0.07)$ \\
\hline
\end{tabular}




\section{Response Times}

RTs to related targets were analysed in a 2 (Training: negative vs. benign) x 2 (Target Valence: negative vs. benign) mixed ANOVA. No significant effects were observed. Notably, the interaction between Training and Target Valence did not approach significance $F(1,69)=.22, p=.64, \eta_{p}^{2}=.01$, indicating that valenced, but unambiguous, scenarios did not alter an interpretation bias. While the pattern of a benign advantage was apparent in the Response Time data (see Table 9), it did not reach significance, $p=0.33$.

\section{Accuracy}

Accuracy (hits) was similarly analysed in a 2 (Training: negative vs. benign) x 2 (Target Valence: negative vs. benign) mixed ANOVA. No significant effects were observed. Median $d^{\prime}$ scores were analysed similarly to RT in a 2 (Training: negative vs. benign) x 2 (Target Valence: negative vs. benign) mixed

ANOVA. There was a main effect of valence, $F(1,69)=28.15, p<.001, \eta_{p}^{2}=.28$. Participants were better able to discriminate between related and unrelated words when that target was benign $(M=2.62, S D=.56)$ than when the target was negative $(M=2.23, S D=.56)$. No other significant effects were observed.

The results of Experiment 4 suggest that comprehending valenced unambiguous scenarios and completing related, valenced word fragments was not sufficient to alter an interpretation bias. Following training, participants were not faster to make relationship judgements to training congruent targets relative to training incongruent targets. The failure to find evidence of an interpretive bias following training argues against an Emotional Priming Effect as repeatedly accessing or activating negative or benign emotional material in the training phase did not facilitate the selection of meanings on a later priming task. If an emotional priming effect was responsible for inducing a training-consistent interpretive bias then effects should be strongest following the manipulation in Experiment 4, due to the explicitly valenced information presented within the training phase relative to all other experiments.

A benign bias was observed in accuracy and a trend towards similar effects in response times was observed. It appears that there is a stable advantage for benign over negative targets in a relationship decision across all experimental 
conditions regardless of training condition.

\section{General Discussion}

Interpretive biases only emerged in Experiment $1 \mathrm{a} / \mathrm{b}$, when ambiguity resolution was a feature of the training paradigm. These results coupled with a failure to find interpretation bias effects in Experiments 3 and 4 support an Ambiguity Resolution Account of CBM-I, and argue against an Emotional Priming Account. It appears that over the course of the training phase participants learned a production rule that dictated their interpretation of novel emotional ambiguity. Training appears to act as a global context, which directly and specifically impacts the selection of meanings of emotionally ambiguous stimuli rather than broadly activating a valenced category and enhancing responses to negative information in a more general manner.

Similar findings were reported by Clarke and colleagues (2014) who used a delayed recognition memory paradigm to test the emergence of an interpretation bias following training with and without the requirement for ambiguity resolution. Because delayed tasks reflect both selection and post-lexical processes, it was possible that ambiguity training in their paradigm affected postlexical (i.e., memory, decision, and response processes) and not selection processes. However, the present studies used an online priming task at test with comparable results to Clarke and colleagues (2014), arguing that ambiguity resolution during training is essential for biasing meaning selection. These findings support Clark and Colleagues' (2014) conclusions that an Ambiguity Resolution Account explains the observed changes in interpretation following CBM-I training.

However, two previous studies using a semantic priming task at test have found evidence that supports an Emotional Priming Account, which is in contrast to my own findings (Hoppitt et al 2010a; Grey \& Mathews, 2009). There are two possible explanations for this discrepancy, both related to task demands. First, Hoppitt et al.'s (2010a) and Grey and Mathew's (2009) studies used single word stimuli across both training and test phases. During training, Grey and Mathews (2009) asked participants to judge the relationship between primes and targets while Hoppitt et al (2000a) asked participants to solve word fragments following single word primes. In the test phase of both studies, participants made lexical 
decisions to targets that were primed by homographs. In both studies lexical decisions to training-congruent interpretations of the homographs were facilitated regardless of whether the training involved ambiguity resolution or not. The procedural overlap in both studies may have resulted in the generalisation of certain cognitive processes or strategies across phases.

A second possible explanation for the discrepancy between my own results and those of Hoppitt et al. (2010a) and Grey and Mathews (2009) is the use of different tasks in the test phase. Both studies that have found evidence for an Emotional Priming Account have used a primed lexical decision task during the test phase. Lexical decision tasks have been known to be affected by basic orthographic and phonological factors and are likely to index an early stage of lexical access that may be more susceptible and sensitive to emotional priming effects. On the other hand, semantic relationship judgements are reliant on semantic information and therefore necessarily reflect a later stage of lexical processing (Hino, Lupker \& Pexman, 2002). A relatedness decision was selected for all experiments so far, in order to force interpretation of the ambiguous prime (Balota \& Paul, 1996). However, in Chapter 5, I will determine whether similar training effects are observed using a lexical decision task at test. 


\section{Chapter 5: Testing Modification of Interpretation with a Lexical Decision}

CBM-I research has often relied on semantic priming tasks to assess the interpretation of emotional ambiguity following training. Two priming tasks have been used in the CBM-I test phases to date, a semantic judgement task (as used here in Experiments 1-4) and a lexical decision task. Semantic judgement tasks require participants to assess the relationship between a prime word and a target word. Alternatively, during a lexical decision task, participants see a prime word followed by a string of letters and must indicate whether the string is a word or a nonword. In the CBM-I literature, lexical decision tasks and semantic judgement tasks have been used interchangeably to assess the presence of an interpretation bias. However, there are a number of key differences between these two tasks that may reflect different strategies adopted by a participant in order to produce a correct response.

A semantic relationship judgement and a lexical decision require different decision processes and participants may rely more heavily on different stages of lexical processing in order to complete a given task. In a relatedness judgement, the participant must activate and then select a meaning of both the ambiguous prime and the target word. Following this, they must compare the two meanings for semantic relatedness before making a response. Alternatively, in a lexical decision task the participant needs only to activate the lexical entry of the target word to correctly judge that it is a word. While lexical decisions do reflect the recruitment of semantic information (as seen by semantic priming effects on task performance), the task is much more sensitive to lexical factors than a semantic relationship judgement which necessarily draws on semantic information. The tasks may therefore lead to the adoption of different strategies. Participants doing the semantic judgement task would be more likely to engage a strategy that considers the relationship between words (which is reliant on selection of a prime meaning) than participants in the lexical decision task (which is reliant on activation of a particular meaning beyond a word identification threshold).

Furthermore, lexical decisions are sensitive to a number of factors that do not affect responses in a semantic relatedness task. These include orthographic properties such as length (e.g., New, Ferrand, Pallier \& Brysabaert, 2006) and experimental conditions such as the proportion of trials that are related to the 
prime (Neely, Keefe \& Ross, 1989). This suggests that lexical decisions are more sensitive to early lexical effects and involve shallower semantic processing, whilst semantic judgements reflect the adoption of explicit strategic processes driven by later context effects.

Results from the use of lexical decision tasks within the CBM-I literature have been mixed. The first study to use a lexical decision task at test was conducted by Grey and Mathews (2000) in their Experiment 2. During the training phase participants saw ambiguous primes (e.g., sack), followed by word fragments which, when completed, were related to the benign (e.g., bag) or negative interpretation (e.g., job) of the prime. In the test phase, participants saw ambiguous primes and completed lexical decisions to related negative and benign target words. Participants made faster lexical decisions to trainingcongruent words, which the authors argued indicated that lexical access was biased towards meanings corresponding with the training valence.

In their Experiment 3 participants completed a training phase in which the order of the homograph prime and the related fragment words were reversed. This time participants saw word primes (e.g., bag/job), followed by a homograph target (e.g., sack). The test phase was the same as Experiment 2. The results mirrored those of Experiment 2 and the authors concluded that training with ambiguity resolution was not necessary in order to alter an interpretation bias at test, consistent with an Emotional Priming Account. However, the authors did not have a way of calculating a priming measure to determine whether responses in the test phase were facilitated to all negative targets. They therefore could not conclude whether participants only showed facilitation to targets following a prime - which would show support for a modified interpretive bias following training.

It has been suggested that biases in interpretation should only occur when there is competition between meanings in the test phase (MacLeod \& Mathews, 1991). It therefore follows that an interpretation bias should only appear on tasks that evoke competition between meanings (such as following the presentation of a homograph) rather than following the presentation of unambiguous words. Grey and Mathews (2009) therefore followed up their Experiment 3 (Grey \& Mathews, 2000) by removing the presence of ambiguity 
during the test phase. They predicted that if participants showed facilitated response times to targets following unambiguous primes then this would support an Emotional Priming Account, as this would be evidence of general facilitation for processing of all training congruent stimuli. Participants completed the same training as in Experiment 3 in Grey and Mathews (2000). Following training, participants completed a lexical decision task for negative and benign words following scrambled nonword primes. Because they used nonword primes in the test phase, a training congruency effect would reflect activation of similarly valenced targets, not priming by the ambiguous homograph. No significant interaction was observed between training condition and target valence in lexical decision times. The authors concluded that without competition between meanings during the test phase, interpretation biases did not emerge. Therefore, biases in the interpretation of ambiguity only occurred when participants responded to targets that followed an ambiguous prime and not those that followed a nonword prime.

One other study has used lexical decisions in a test phase following CBM-I training. Hoppitt et al. (2010b) used an active and a passive training condition to examine the effects of training on lexical decisions at test. During training participants completed a word fragment completion task preceded by either ambiguous or unambiguous primes e.g.,

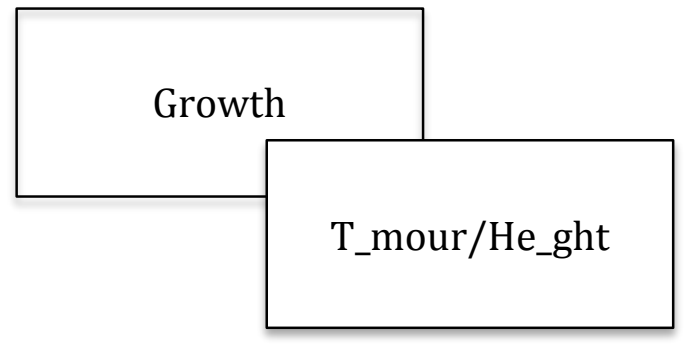

Active Training

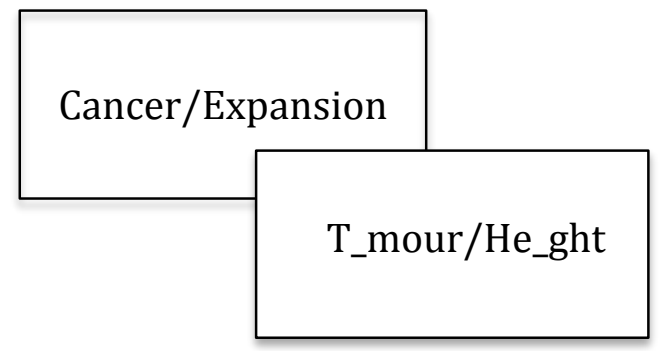

Passive Training

Figure 7. Active and Passive Training Conditions in a CBM-I training phase (adapted from Hoppitt et al., 2010b).

During the test phase participants completed lexical decisions to word and nonword targets that were presented following cued (word) or uncued (XXXXX) primes. Cued primes were ambiguous words with one negative and one 
benign meaning (e.g., patient) and word targets were either negative (e.g., hospital) or benign (e.g., kind) interpretations of the ambiguous prime. No significant effects of training were observed on uncued trials, which is consistent with Grey and Mathews' (2009) finding that training did not produce faster responses for training-congruent words presented after nonword primes. A significant interaction between target valence and training valence was found in the cued trials. The authors found that although lexical decisions were faster overall for benign targets relative to negative targets, this difference was smaller in the negatively trained condition. What the authors failed to do was compute a priming measure whereby responses to cued trials could be directly compared to responses on uncued trials, to calculate the amount of priming produced by the homographs as a function of training.

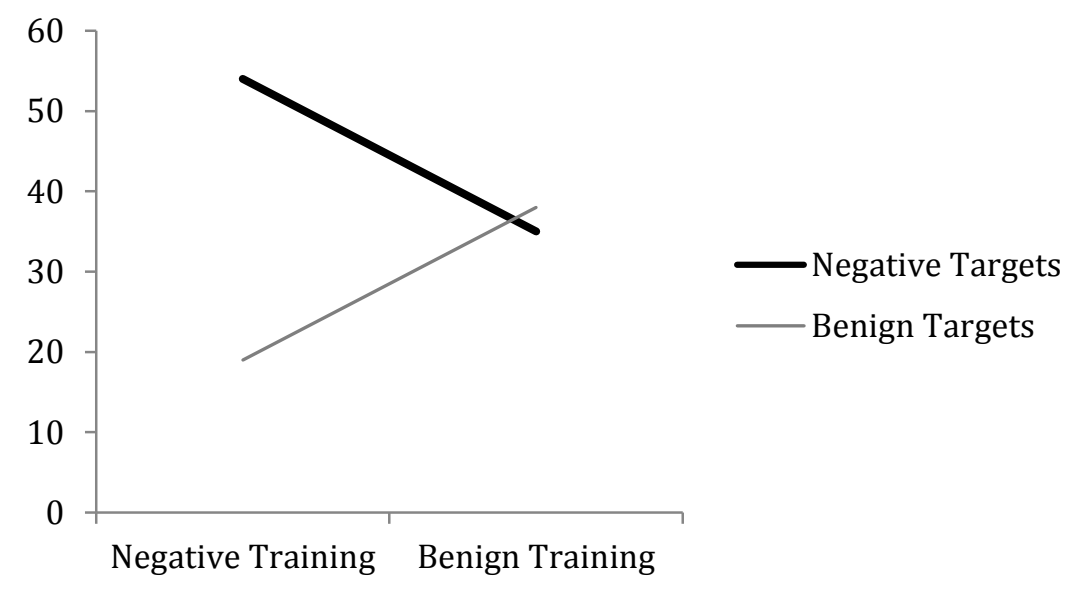

Figure 8. Priming for Negative and Benign Targets (Uncued trial response time

- Cued trial response time) as a function of Training Valence (Negative or Benign Training). Adapted from Hoppitt et al., (2010b).

However, a priming measure can be calculated from their data by subtracting the mean lexical decision times for cued trials from uncued trials. In Figure 8, positive values indicate faster response times for cued over uncued trials (i.e., priming) in that particular training and target condition. What becomes clear is the large amount of priming for lexical decisions to negative relative to benign targets following negative training. Therefore the results suggest that regardless of whether participants were required to resolve ambiguity during negative training, they were faster to call negative targets 
words relative to benign targets when primed by an ambiguous target.

Taken together, Hoppitt et al.'s (2010b) and Grey and Mathews (2000, 2009) results may appear confusing as they suggest that ambiguity resolution during training is not required in order for interpretive biases to occur in the test phase, which is in line with an Emotional Priming Account. Furthermore, trainingcongruent biases only emerged in the test phase when participants were required to make lexical decisions to targets following an ambiguous prime, which is in line with an Ambiguity Resolution Account.

It is important to note that an Emotional Priming Account and an Ambiguity Resolution Account are not necessarily mutually exclusive. It is quite possible that both accounts are valid yet their effects may be observed using different priming tasks. It is possible that lexical decisions are more sensitive to transient emotional effects, which may drive responses to training-congruent valences, like an Emotional Priming Account suggests. On the other hand, a semantic relationship judgement recruits greater strategic processing and may therefore be more sensitive to the induction of a learned processing rule, in line with an Ambiguity Resolution Account. Another important consideration is the methodology of the previous lexical decision tasks used in CBM-I studies to date.

One issue concerns the operationalization of the lexical decision task and the specific methodology employed by different researchers. First, lexical decisions are much more vulnerable to orthographic and pre-semantic features of words such as length (e.g., Balota \& Chumbley, 2006). Neither study balanced features such as length, which may have led to item-specific variations between experiments. Secondly, each study used a different type of prime on uncued trials. Whilst Hoppitt et al (2010b) used a series of XXXXX's on their uncued trials Grey and Mathews (2009) simply rescrambled existing homograph primes into non-word primes. This is problematic for two reasons. First, without piloting it is hard to say whether these words were actually treated as non-words by participants. As they were relatively short words, it is possible that the scrambled primes resembled the original words enough to act as actual word primes (E.g., scrambling sack to sakc). Alternatively, the nonword prime facilitation effect suggests that words, or stimuli resembling words may serve to "warm up" a lexical processor prior to the presentation of a target therefore 
disadvantaging targets preceded by a series of X's (Forster, 1981). In this way using scrambled words as uncued stimuli may have unintentionally primed the targets, which may explain the sub-threshold appearance of emotional priming effects in Grey and Mathews (2009) results.

Additionally, the overlap between the training and test phases may benefit performance and may serve to result in a person adopting a particular strategy when completing the task that results in facilitated performance over time rather than reflecting the training of an interpretation bias.

Questions now arise as to whether training affects the strategies adopted by participants in different manners, depending on the task used in the test phase. It is possible that different tasks used at test tap different processes, some of which may be more influenced by training than others. Based on the results from my previous experiments, CBM-I actively changes the selection of a single meaning from an ambiguous word, at least when participants are required to perform a semantic relatedness decision. The next question is whether these biases also emerge on a lexical decision task, which assesses the earlier stage of lexical access. Alternatively, the appearance of an interpretive bias on a lexical decision task in other studies may reflect methodological artifacts associated with overlapping training and test phases or a failure to balance stimuli. The studies reported in this thesis so far provide evidence for an Ambiguity Resolution Account over an Emotional Priming Account, although as noted before the two accounts are not mutually exclusive and are therefore not in competition with one another. An Emotional Priming Account predicts training effects on both cued and uncued trials, because activation of valenced information during training should facilitate access to valenced words at test, regardless of the presence or absence of the ambiguous homograph. However, an Ambiguity Resolution Account predicts that training effects should only be seen on trials cued by a homograph, as training should only affect the interpretation of an ambiguous prime.

\section{Experiment 5}

The aim of Experiment 5 was to assess the induction of an interpretation bias using the same CBM-I training paradigm as used in previous experiments, but coupled with a primed lexical decision task at test. Participants completed 
the scenario-based training phase used in Experiments 1a. In the test phase, participants saw word and nonword targets following primes, which were either emotionally ambiguous words, or a series of XXXXX's. Targets were either negative or benign words related to the emotionally ambiguous primes, or they were scrambled nonwords. Uncued trials allowed me to compute a priming measure in order to assess whether training altered an interpretation bias that affected responding to primed relative to unprimed targets.

\section{Method}

\section{Participants}

Seventy-three undergraduate students (28 men, 45 women; mean age: 20.7 years) participated for course credit. All were native English speakers with normal or corrected-to-normal vision. Participants reported no current or previous diagnoses of depression. They were randomly assigned to receive either negative or benign training. The study was approved by the Human Ethics Committee, Victoria University of Wellington. All participants gave written informed consent prior to participation.

\section{Stimuli and Apparatus}

The scenarios used in the training phase were identical to those used in Experiment 1a. During the test phase participants saw prime-target pairs and made a lexical decision to the target word. Prime-Target pairs for the test phase were the same as the pairs used in Experiment 1 however, in half of the trials participants saw a nonword preceded by a prime. Construction of nonword targets is detailed below.

\section{Nonword Targets}

Nonwords were created by inputting all word targets from Experiment $1 \mathrm{a}$ into a pseudoword generator (Keuleers \& Brysabaert, 2010). This process allowed for generation of nonwords that matched real words on a number of orthographic factors such as length, orthographic neighbourhood, bigram frequency, Orthographic Levenshtein Distance and Neighbourhood size (Balota \& Yap, 2006; Balota \& Chumbley, 1984). After construction, the selected nonwords were balanced so that each nonword was matched on these properties with its original word. This allowed for the inclusion of nonwords which did not differ on any key properties relative to its original word form. 
Table 10 shows a typical spread of word and nonword targets for a particular ambiguous prime word stalk. During the test phase participants saw a prime followed by a target. There were two prime conditions, Cued (e.g., stalk) and uncued (e.g., $X X X X X$ ) and two target conditions (word and nonword). Word targets were related to the ambiguous prime and were either negative (e.g., creep) or benign (e.g., flower) in valence. Nonwords were the matched psuedowords generated from the negative and benign target words.

For the Cued condition the 52 emotionally ambiguous prime words from Experiment 1 were used. The Uncued condition simply consisted of 52 strings of X's. Each prime was matched with a word target and a nonword target (See Table 5). Counterbalancing for the Cued trials meant that if Participant 1 saw the ambiguous prime Stalk paired with its negative interpretation Creep then Participant 2 would see the same prime paired with its benign interpretation Flower. This allowed for an equal distribution of valenced prime-target pairs across the experiment and made sure that participants were exposed to each meaning of an ambiguous word, once following a homograph prime and once following a series of XXXXX's.

\section{Table 10.}

Example of counterbalancing across participants. Typical trials across two participants as a function of list.

\begin{tabular}{cccccc}
\hline & Prime Type & Prime Example & Target Type & Target Valence & Target Example \\
\hline \multirow{2}{*}{$\begin{array}{c}\text { Participant 1 } \\
\text { (List 1). }\end{array}$} & Cued & Stalk & Word & Negative & Creep \\
\cline { 2 - 5 } & Uncued & XXXXX & Nonword & Benign & Flimer \\
\cline { 2 - 5 } & \multirow{2}{*}{ Cued } & Stalk & Word & Benign & Flower \\
\cline { 2 - 5 } Participant 2 & & Word & Benign & Flower \\
(List 2) & \multirow{2}{*}{ Uncued } & \multirow{2}{*}{ XXXXX } & Nonword & Negative & Croop \\
& & & Nonword & Benign & Flimer \\
& & & Word & Negative & Creep \\
\hline
\end{tabular}


This pairing of primes and targets yielded three measures. One is response times to cued targets - do participants show facilitated lexical decisions to training-congruent targets following an ambiguous cue? This indexes the availability of meanings of an ambiguous word following training. Another measure is lexical decision response times to uncued trials - do participants show facilitated response times to uncued targets in a training congruent manner? This indexes facilitated access to training congruent targets, but says nothing about ambiguity resolution. Because there is no ambiguity in the prime, any observed effects on uncued trials must be caused by emotional priming. The third is a computed uncued - cued measure - how much does the ambiguous prime speed the response to the target relative to an uninformative prime? This measure reflects any semantic priming provided by the interpretation of the ambiguous homograph.

\section{Procedure}

Participants were randomly assigned to receive either negative or benign training. Training was identical to that in Experiment 1a. During the test phase, all participants completed the same primed lexical decision task to assess interpretative biases. An ambiguous prime (e.g., stalk; Cued condition) or a string of letters (e.g., XXXXXXX; Uncued condition) was presented centrally on the screen for $250 \mathrm{~ms}$ followed by a target that was either a related negative or benign word (e.g. , creep or flower) or a nonword (e. g. , flimer or croop) matched to the related word. The time between the onset of the prime and the onset of the target was $750 \mathrm{~ms}$. Participants indicated whether the target was a word or a nonword by pressing the 1 or 2 key on the number pad of the keyboard. Participants responded to 208 trials presented in random order; 104 targets were words (52 negative targets and 52 benign targets in each cue condition) and 104 targets were nonwords (52 converted from negative related words and 52 converted from benign related words). The target remained on screen until the participant made a response, and was followed by an inter-trial interval of $1000 \mathrm{~ms}$. The between-subjects variable was training condition (negative or benign), and the within-subjects variables were prime type (Cued or Uncued) and target valence (negative or benign). 


\section{Results}

Accuracy and mean RTs were calculated as in Experiment 1, and are reported in Table 11. The mean time taken to correctly complete word fragments in the training phase was $2364 \mathrm{~ms}$ and the mean overall accuracy was $94 \%$. Four sensitivity scores were calculated for each participant; their $d^{\prime}$ scores, which in this experiment is their ability to discriminate between a word and a nonword, to cued and uncued negative and benign targets. A criterion for participant inclusion was established so that any participant with a d' score of greater less than one in at least one condition was removed. Three participants were removed from data analysis. There were then 36 participants in the Negative Training Condition and 34 in the Benign Training Condition.

\section{Response Times}

Response times (RTs) to target words were analysed in a 2 (Training: Negative vs. Benign) x 2 (Target Valence: Negative vs. Benign) x 2 Prime Type (Cued or Uncued) mixed analysis of variance (ANOVA) with Training as a between-subject variable and Target Valence and Prime Type as a within-subject variable

There was a main effect of cue type $F(1,68)=13.39, p<.001, \eta_{p}^{2}=.16$, participants were faster to make lexical decisions to targets preceded by a prime $(M=712 \mathrm{~ms}, S D=162)$ than to targets preceded by a series of X's $(M=779 \mathrm{~ms}, S D$ $=169$ showing that the homograph primes did facilitate lexical decisions. There was a main effect of target valence $F(1,66)=40.22, p<.001, \eta_{p}^{2}=.37$, participants were faster to call benign targets words $(M=734 \mathrm{~ms}, S D=168)$ than negative targets $(M=758, S D=169)$. This mirrors the benign advantage seen for targets in previous experiments. No other significant effects were observed. Notably, there was no training valence $\mathrm{x}$ target valence interaction, $\mathrm{F}=.001 \mathrm{p}=.98$, nor a training $\mathrm{x}$ target $\mathrm{x}$ cue type interaction, $\mathrm{F}=1.15, \mathrm{p}=.29$. Therefore the training phase did not induce changes in interpretive bias. 
Table 10

Mean Response Times (ms) and Accuracy of Subjects' Responses to Targets as a Function of Training for Experiment 5(with Standard Deviations in Parentheses)

\begin{tabular}{|c|c|c|c|c|}
\hline \multirow[b]{2}{*}{ Training Valence } & \multicolumn{2}{|c|}{ Absent Prime } & \multicolumn{2}{|c|}{ Ambiguous Prime } \\
\hline & Negative & Benign & Negative & Benign \\
\hline & \multicolumn{4}{|c|}{ Response Times (ms) } \\
\hline Negative & $816(204)$ & $802(213)$ & $756(203)$ & $726(196)$ \\
\hline \multirow[t]{2}{*}{ Benign } & 764 (112) & $732(107)$ & $690(104)$ & $671(106)$ \\
\hline & \multicolumn{4}{|c|}{ Accuracy } \\
\hline ' & $.97(0.03)$ & $.96(0.4)$ & $.98(0.3)$ & $.98(0.3)$ \\
\hline Benign & $.98(.03)$ & $.97(.05)$ & $.98(.03)$ & $.98(.03)$ \\
\hline
\end{tabular}

The lack of interactions with cue-type show that priming was observed equally in all conditions.

\section{Accuracy}

Accuracy to correctly judge targets as words was analysed in a 2 (Training: negative vs. benign) x 2 (Target Valence: Negative vs. Benign) x 2 Prime Type (Cued or Uncued) mixed analysis of variance (ANOVA) with Training as a between-subject variable and Target Valence and Prime Type as a withinsubject variable. No significant effects were observed

\section{General Discussion}

The results of Experiment 5 do not support the induction of a trainingcongruent interpretation bias on a lexical decision task following CBM-I training. Participants were not faster to make lexical decisions to target words that were emotionally congruent with their training phase. A typical priming effect was observed in response times following training as participants were faster to make lexical decisions on cued trials relative to uncued trials. However, this 
priming benefit did not extend to affective processing of the target. Therefore, spreading activation between the word cues and word targets resulted in facilitated lexical decision times relative to uncued targets but not in a manner biased by training valence.

Further investigation revealed that, on both cued and uncued trials, there was an overall advantage for benign targets in response times. This is interesting as one possible interpretation of the benign bias in previous experiments was that the benign relationship decision was an easier one. The more likely conclusion now is that benign words are simply easier to read than negative words. The fact that the benign advantage was equivalent for both cued and uncued trials argues against the possibility that participants had a bias to interpret the ambiguous homograph benignly, but rather indicates that they processed the benign words more quickly. Negative words appear to have a processing disadvantage relative to neutral words in other paradigms (e.g., Bradley, Mogg \& Williams, 1994; Challis and Krance, 1988; Ruiz-Caballero \& Moreno, 1992; Nakic et al., 2006). It is thought that highly arousing negative information is so salient that it captures attention and disadvantages behavioural responses relative to neutral words (Carretie, Martin-Loeches, Hinojosa \& Mercado, 2008).

It is likely that the differences in task demands during the test phase are responsible for the observation of an interpretive bias in previous experiments but not here. The lexical decision task is concerned with judgements of lexical form whilst a semantic judgement task is concerned with meaning. It is possible that the different task demands encouraged participants to adopt different strategies in order to complete the tasks. The relationship decision requires a person to interpret the ambiguous prime in order to make a correct response, while the lexical decision task asks participants to merely respond to the target. The relationship decision therefore forces the participant to select a meaning for an ambiguous word before they can respond. Participants in each experiment may therefore be using the emotional training context in different ways in order to complete the task. In the relationship decision task participants may use emotional context to guide the selection of an appropriate meaning from the ambiguous prime. However, in the lexical decision task, participants are not 
drawing on the available context to make decisions about letter strings.

Interestingly, Hoppitt et al. (2010b) found effects of training on a lexical decision task and their results stand in contrast to my own research. However, they state that they did not find evidence of an interpretation bias "as it is generally understood". Participants in the negative training condition were faster to name negative targets regardless of whether the target was preceded by a prime or not. The opposite pattern of results was found for participants in the benign training condition. The authors also found that similar results were obtained regardless of whether participants were required to resolve ambiguity during training or not. It is possible that repeated activation of valenced information has a transitory effect on similarly valenced information that does not extend to the actual interpretation of emotional ambiguity. However, Hoppitt et al.'s task (2010b) involved a high degree of overlap between their training and test phases. This could potentially have resulted in participants adopting a strategic processing style which facilitated responses to word stimuli in a manner that led to the appearance of an interpretation bias. Using procedurally distinct training and the test phases so that the same strategies could not be employed, I was unable to demonstrate training-congruent facilitation on a lexical decision task, as seen in Hoppitt et al. (2010b).

Unlike the SOA experiment in Chapter 2 that directly looked at the time course of access and integration processes, the lexical decision task can tell us about the effect of emotional context on the likely strategy employed by a participant. It appears that on this task, emotional context did not interfere with the availability of meanings and a participant's ability to respond to a letter string following an ambiguous cue. In this case emotion served as a superfluous context rather than an informative semantic indicator. Instead it appears that in order to obtain training-congruent responding on a task at test, it must require that the participants interpret ambiguity. 


\section{Chapter 6: General Discussion}

Cognitive Bias Modification for Interpretation has been a growing area of research for over a decade (Grey \& Mathews, 2000; Mathews \& Mackintosh, 2000; Hallion \& Ruscio, 2011; Mene-Lothmann, et al., 2014; Yiend, 2004;). Over this time, a number of studies have furthered our understanding of the fartransfer effects of training (Beard \& Amir, 2008; Mackintosh, Mathews, Yiend, Ridgeway \& Cook, 2006; Salemink, van den Hout \& Kindt, 2009; Wilson, MacLeod, Mathews \& Rutherford, 2006), which has been important for elucidating the relationship between interpretive biases and mood effects. However, less research has been devoted to understanding the near-transfer effects of training (e.g., Hoppitt et al., 2010a, Hoppitt et al., 2010b, Clarke et al., 2014), which is necessary if we are to discriminate between possible mechanisms underlying induced interpretive change. Understanding the mechanisms that support CBM-I effects can also give us insight into the cognitive processes underlying meaning sense disambiguation more generally (Koster et al., 2009).

The overarching aim of the series of studies in this thesis was to develop and systematically modify a novel CBM-I procedure in order to explore the underlying mechanisms of emotional interpretation change. I examined a number of key features known to affect lexical ambiguity resolution in order to understand their effects on interpretive biases. Specifically, I examined the timecourse of the emergence of interpretive biases, the role of emotional context in guiding the selection of an appropriate meaning, and the types of strategies employed by participants as a function of task constraints. More broadly, I asked the question, what role does emotion play in the interpretation of ambiguous language?

In Chapter 2, a novel paradigm was developed and administered to shift an emotional interpretation bias in either a negative or a benign direction. The training paradigm was designed in order to overcome two common limitations of CBM-I paradigms. First, an online probe of interpretation at test was used to minimize the potential for effects to arise through post-lexical memory or decision processes, which can be recruited in delayed tasks. Second, the procedural overlap between the training and test phase were minimised in order 
to reduce the transfer of procedural factors from training to test. The training phase differed from traditional scenario-based CBM-I phases in that the word fragment was not a sentence-final word, instead it was an associate of either the negative or benign interpretation of the scenario. This means that all of the training sentences remained ambiguous, and it was hypothesised that the consistently valenced fragments would induce training congruent biases in interpretation. Following training, participants were faster to make relationship judgements to training-congruent targets following ambiguous word primes. The interpretive bias observed following training is similar to interpretive biases following previous scenario-based training phases, suggesting the training phase did encourage interpretation of the ambiguous scenarios. The effect was further replicated in Experiment 1b, which bolsters evidence for the successful modification of an interpretive bias following scenario-based training.

Chapter 3 shortened the SOA in the test phase in order to shed light on the time course over which biased interpretive processes arise following training. Decreasing the temporal separation of the prime and the target made it possible to present the target to participants before the selection of a single meaning had occurred. No differences in relatedness decision response times were found as a function of training in the short SOA condition. The results suggest that, rather than affecting the initial activation of each meaning, the training phase alters the selection of an appropriate meaning from amongst alternatives at a later stage.

In Chapter 4, the training paradigm was systematically modified in order to isolate the critical features responsible for later changes in the interpretation of emotional ambiguity. Interpretive biases were not altered through training when participants were required to repeatedly access emotional information by simply completing negative or benign word fragments (Experiment 3). Interpretive biases were also not shifted when participants completed word fragments related to negative or benign unambiguous sentences (Experiment 4). It appears that the repeated resolution of emotional ambiguity during training (Experiments 1a \& 1b) was essential in order to modify an interpretive bias at test. These results are in line with an Ambiguity Resolution Account, which states that participants learn an implicit procedural strategy during training, and 
implement it when interpreting novel emotional ambiguity.

Finally, Chapter 5 investigated the effects of interpretation training when participants were required to complete a lexical decision in the test phase. No differences in lexical decision response times were observed as a function of training, indicating a failure to modify interpretive biases. The scenario-based training phase only served to guide meaning resolution processes when the task required participants to interpret the prime. These results support the idea that emotional context only biased meaning selection when explicit interpretive strategies were encouraged in the test phase.

Figure 9 shows the observed bias in the test phase across all experiments. Rightward values indicate a negative bias, and leftward values indicate a benign bias. One noticeable feature is the stable benign advantage across all benign training conditions. Regardless of experimental condition, participants in benign training conditions show a response time advantage for benign interpretations of emotionally ambiguous words. This stability in the benign bias across experimental manipulations suggests that the experiments are reliably tapping interpretive processes. One possible explanation for the benign bias could be that benign training does not modify an interpretive bias; instead participants' responses may reflect a general advantage for benign over negative words in the test phase. Most apparent in the figure is the effect of negative training on modifying interpretive biases in Experiments 1a and 1b. Only when participants were required to complete word fragments which disambiguated scenarios did an advantage for negative interpretations emerge in the test phase.

\section{Implications for Mechanistic Accounts of Modified Interpretive Biases}

The two mechanisms highlighted for investigation in this thesis were those of Ambiguity Resolution and Emotional Priming. These accounts are not mutually exclusive so it is important to consider the evidence for Emotional Priming and Ambiguity Resolution in turn. 


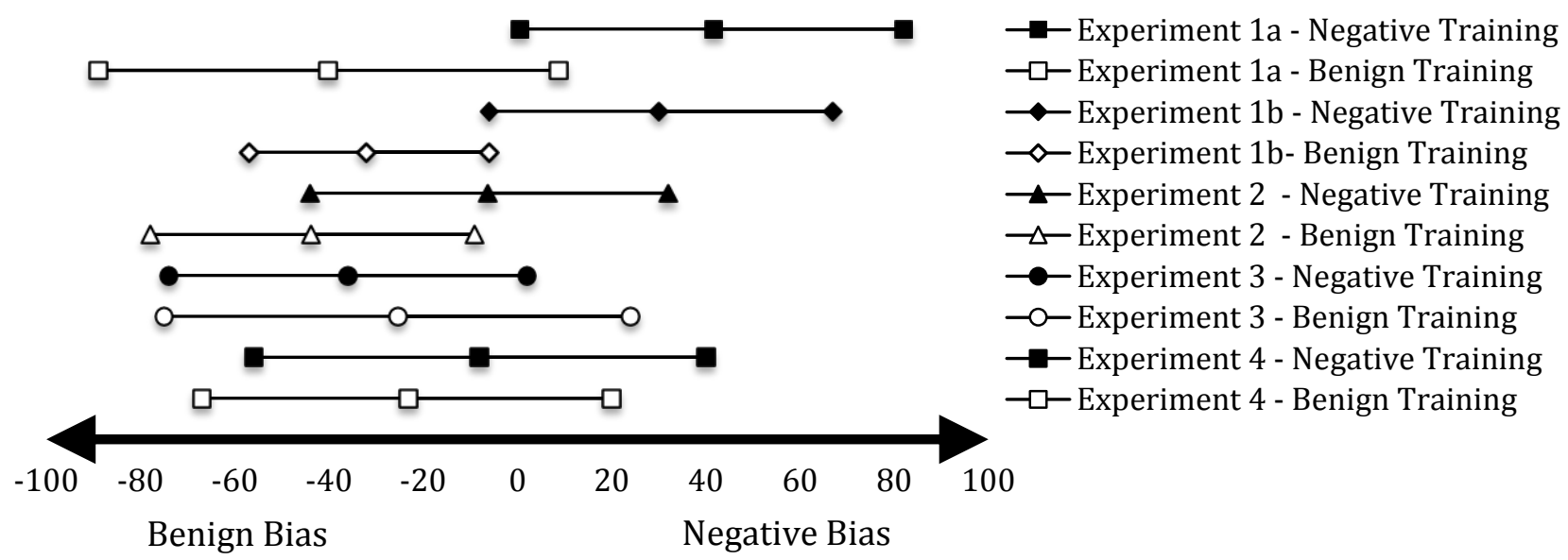

Figure 9. Mean response time advantage (and 95\% Confidence Intervals) for Negative Related Targets relative to Benign Related Targets as a function of Training Condition and Experiment.

According to an Emotional Priming Account, interpretive bias effects arise when emotional information in the training phase broadly activates similar concepts within the semantic network. In this way differences in interpretation patterns could emerge at early meaning activation stages or later meaning selection stages, as training may bias the relative availability of meanings in a training-congruent manner. In Experiment 2, when the SOA between the prime and the target was shortened, no interaction with training was observed. Here, participants were not faster to interpret training-congruent meanings of ambiguous words as a function of the training paradigm. These findings argue against an early Emotional Priming account and suggest that interpretive differences only emerge at a later meaning selection stage, rather than an earlier meaning activation stage. Furthermore, in Experiment 5, no differences emerged when participants were required to make lexical decisions to negative and benign interpretations of emotionally ambiguous words. This further argues against a lexical activation account and highlights the role of selection (as required by a semantic task) over activation. The Emotional Priming account further posits that repeatedly accessing emotional information in the training phase is the critical factor in modifying an interpretive bias. Therefore, if 
emotional priming could explain changes in interpretation following training, then bias shifts should be evident following any training condition that involved the repeated presentation of emotionally valenced stimuli. The results of the two experiments reported in Chapter 4 argue against this possibility. When the relationship between the training scenarios and the word fragments was removed so that participants simply completed negative or benign word fragments, training did not serve to modify interpretive biases. Furthermore, interpretive biases were not observed when participants read strongly valenced sentences and completed negative or benign word fragments (the most explicitly valenced condition; Experiment 4). If interpretive bias modification can arise through emotional priming, then biases should have been modified by the emotional information in the fragments (Experiment 3) or in the unambiguous sentences (Experiment 4).

According to an Ambiguity Resolution Account a participant learns to apply an implicit production rule when encountering novel instances of emotional ambiguity. The Ambiguity Resolution Account predicts that changes in interpretation should only be evident at a later meaning selection stage rather than an earlier meaning activation stage. In Experiment 2, when the SOA between primes and targets was shortened, no differences in relationship decision times were noted. Furthermore, in Experiment 5, when participants were required to make lexical decisions to negative and benign interpretations of ambiguous words, no interaction with training was observed. These findings are in support of an Ambiguity Resolution Account as they suggest that changes in interpretive biases are only observed at a later selection stage where participants are required to interpret the ambiguous prime word. The Ambiguity Resolution Account further predicts that changes in meaning section should only be observed when a participant is trained to resolve ambiguity during training. No changes in interpretive bias were observed when participants simply completed valenced word fragments (Experiment 3) or when they read highly descriptive, unambiguous emotional scenes (Experiment 4). These findings are in line with an Ambiguity Resolution Account, as they suggest that only when participants are asked to repeatedly resolve ambiguity does a bias in later interpretation occur. Lewicki et al. (1988) discuss the induction and later 
production of an implicit procedural rule that might drive the appearance of an interpretation bias in an 'IF /THEN' manner. According to this account, the repeated pairing of certain events (in this case an ambiguous scenario and a valenced word) results in an implicit association between the two events and encourages the later interpretation (via an encoding algorithm) of further events in a similar manner (for a discussion on the cognitive architecture of procedural knowledge see Budiu \& Anderson, 2004). However, this implicit procedural rule was not discussed as being specific to the interpretation of ambiguous stimuli. In fact, procedural rules are thought to reflect a broad ability of the cognitive system to detect patterns and implicitly learn relationships between features and events. In this case, we should have observed similar patterns in Experiment 4, which similarly trained people to make associations between negative or benign scenarios and emotionally related word fragments. Why then is this effect only observed on a task that trains the explicit interpretation of emotional ambiguity? One possibility is that the CBM-I task in Experiments $1 \mathrm{a}$ and $1 \mathrm{~b}$ trained a much more specific rule than has been seen in previous encoding algorithm studies (e.g., Lewicki, Hill \& Boss, 1989).

In this thesis, interpretive biases were only shifted when the training and the test phases were matched on task purpose e.g., activate possible meanings in the presence of ambiguity and then select one candidate. In this way, the training phase may have encouraged the adoption of a particular strategy which only guarantees success if the stimuli in the training phase are also ambiguous and the task requires interpretation of the prime. This would certainly explain the failure to find training effects in Experiment 5, when interpretation of the prime was not necessary.

One interesting implication of the ambiguity resolution account is that emotion is not a critical component of training - rather it simply acts as another source of semantic information. Theoretically then the training phase may not be limited to the modification of emotion-specific interpretation biases, and an interpretive bias could conceivably be shifted by training an unemotional production rule. For example, using a similar training phase as Experiment 1 participants could read ambiguous scenarios describing an unidentified object coupled with associated word fragments related to living and non-living objects. 
Following training, participants could classify homograph primes with one living and one non-living interpretation (e.g., Bat) to examine whether a non-emotional implicit production rule had been trained. Such a demonstration would strengthen the conclusion that a similar training mechanism is at work in CBM-I.

One way that emotion may be considered to be important regarding interpretive biases is in the field of emotional disorders. While the aim of this thesis was not to examine mood effects of CBM-I, it is clear that mood plays an important role in linking clinical disorders with biased processing and may in part, explain changes in interpretative biases following training. Trainingcongruent changes in depression and anxiety have been documented following many CBM-I studies (Mathews \& Mackintosh, 2000; Mackintosh, et al., 2006; Steinman \& Teachman, 2010). Mediational analyses have revealed that CBM-I training has a direct effect on state anxiety and an indirect effect, via modification of interpretive biases, on trait anxiety (Salemink, van den Hout \& Kindt, 2010). It is possible that changes in state anxiety encourage participants to respond in line with their emotional state. While the results of this thesis suggest no specialized role of emotional context in language, clinically, emotion has relevance in that it affects a set of cognitive processes in a mood-relevant manner. Therefore, further research comparing interpretive processes between healthy and clinical populations will further shed light on the importance of emotion in language processing and psychopathology.

\section{Implications for Theories of Meaning Sense Disambiguation \\ Selection}

Typically, theories of lexical access have focussed on modelling perceptual and activation processes involved in early word identification (Duffy et al., 1988; Twilley \& Dixon, 2000). However, all models either explicitly or implicitly state a role of meaning selection, as this is necessary for word and discourse comprehension, and for communication in general. The predictions generated in this thesis were derived from the Reordered Access account (Duffy et al., 1988) and the Independent Activation Model (Twilley \& Dixon, 2000), both of which posit that meaning sense disambiguation is a two-stage process; activation of all potential meanings, followed by selection of the most appropriate meaning The results of this thesis are consistent with these accounts, which state that upon 
presentation of an ambiguous word both the contextually-appropriate, and the contextually-inappropriate meanings receive activation. This premise is supported by the findings of Experiment $1 \mathrm{a}$ and $1 \mathrm{~b}$ and Experiment 2 . At a short SOA, training context did not affect the activation of meanings. However, at a long SOA, the contextually appropriate meaning received greater activation and therefore responses were faster for training-congruent meanings over trainingincongruent meanings. Overall, these results support the idea that global context provided by training works over time to bias late meaning selection processes rather than the early activation of meaning associates.

\section{Suppression}

Although all meanings of an ambiguous word appear to be activated, only the most appropriate meaning is selected. What happens to the unselected meaning? Although models of ambiguity processing focus on the selection of the appropriate meaning, there has been some discussion around the fate of the unselected meaning. While it is difficult to ascertain what happens to the unselected meaning from the current behavioural tasks, it is possible that training encouraged the suppression of non-selected meanings in Experiment 1a and 1b. Chwilla and Kolk (2003) suggest that inhibition processes are not recruited during a lexical decision due to task demands, and inhibition is only recruited in semantic decisions due to the need to select one meaning over another. In Experiment 5, participants were not actively looking for a semantic relationship between primes and targets and therefore did not rely on an inhibition process in order to make a correct decision. This may explain why interpretive biases were observed in Experiments $1 \mathrm{a}$ and $1 \mathrm{~b}$ and not Experiment 5.

There is no consensus in the literature as to the mechanism or even the existence of suppression processes in meaning selection. However, there are two models which try to explain how inhibition or activation processes may play a role in the final selection of a meaning. According to decay models, context (in this case, the training scenarios) maintains the activation of an appropriate meaning while the inappropriate meaning loses activation over time, ultimately resulting in selection (e.g., Simpson \& Burgess, 1985; Duffy et al., 1988). However, according to inhibition models, the preceding context actively inhibits 
the inappropriate meaning relative to the appropriate meaning, guiding selection of the meaning with the greater activation (e.g., Simpson \& Kang, 1995). It is possible that suppression stems from the training context and either works in isolation or in combination with the selected meaning. In this way the scenariobased training used in the current series of experiments could directly affect the inhibition of the unselected meaning, which fosters the emergence of an interpretive bias. In a relationship task it is difficult to assess what happens to the unselected meaning, because related and unrelated decisions are functionally different processes and therefore cannot be directly compared. One way to assess suppression could be to use ERP, an online measure of cognitive processing, in order to examine the activation levels of different meaning candidates over time and as a function of training. For example, particular ERP components are known to index early emotional recognition of words and the later integration of word meanings into a broader context. Using ERP we could examine the effect that interpretive training has on different aspects of word recognition and meaning selection. For example, does training impact the early evaluation of word arousal or valence, or does it only impact later integration stages where the selection of a word meaning is affected? By using ERP measures to examine cognitive processes as they unfold over time we can examine the relative availability of different word meanings over time and examine processes of activation and inhibition in action.

Importantly, models of meaning selection and suppression altogether ignore the potential role of emotional context as a source of information. In this thesis I was able to demonstrate that repeatedly disambiguating emotionally ambiguous scenarios in a negative or a benign manner facilitated later selection of emotionally-congruent meanings from novel ambiguous words. The results of this thesis suggest that emotion acts as a late-acting context, which guides selection in a manner similar to other forms of semantic information. Therefore, emotion appears to act similarly to other forms of semantic information, in line with Bower's emotional network model (1981). Bower's model conceives emotional information as being coupled with other categories of semantic information in networks, and activation occurs through spreading activation to similar conceptual information. However, whilst the emotional context of the 
training phase increases the relative speed with which an emotionally-congruent meaning is selected, it does not increase the efficiency with which ambiguous words are processed. Participants are not typically more accurate at identifying training-congruent meanings suggesting that training does not serve to totally inhibit one meaning. Instead training influences the speed with which an appropriate meaning is judged as related relative to the inappropriate meaning. Whether this occurs by increasing the activation of an appropriate meaning or by inhibiting the unselected meaning remains to be understood.

\section{Limitations and Suggestions for Future Research}

Far-transfer tasks are essential if we are to demonstrate the ecological validity of CBM-I as a paradigm and reveal its ability to generalise to other areas of emotional reactivity. The main aim of this thesis was not to examine the fartransfer effects of training, such as the emotional outcomes on mood state measures. While this restricts the ability of the current experiments to shed light on the relationship between interpretive biases and psychopathology, the findings point to a future direction for far-transfer studies. One way to extend this research would be to examine the resulting effects of my different training procedures on emotional outcomes in order to determine whether emotional reactivity is impacted in the same manner as interpretive biases. The implicit assumption in all far-transfer studies is that the effects of training on the emotional outcome are mediated by alterations in interpretation (hence the name Cognitive Bias Modification for Interpretation). The results of future studies could have implications for better understanding the different impact of active and passive training tasks on resulting emotional outcomes. All the training phases in this thesis can be considered to be active tasks, as they require the active generation of negative or benign disambiguating words. Previous research suggests that active tasks are a critical feature in the later modification of mood or anxiety (e.g., Hertel \& Mathews, 2011). For example, even though Hoppitt and colleagues (2010a) observed effects of passive training on primed lexical decision (a near- transfer task), only active training affected anxiety when participants later viewed a video of a car accident (a far-transfer task). Similar effects of active training on far-transfer tasks are reported by Hoppitt et al. (2010b) who found that active training in a traditional scenario-based paradigm 
was necessary to alter the emotionality of mental images generated in response to an ambiguous sentence. The results from Experiments $1 \mathrm{a}$ and $1 \mathrm{~b}$ suggest that ambiguity resolution is a necessary feature to induce interpretive change, and not the repeated access to negative unambiguous information. If trainingcongruent mood changes are only observed following the active resolution of emotional ambiguity, like interpretation biases are, this could have far-reaching implications for the development of treatment programmes for emotional disorders and for understanding of the causal relationship between interpretive biases and psychopathology.

Another limitation of my research has to do with the apparent fragility of the interpretive bias effect. Interpretive biases on a relationship decision task were only observed when participants were required to disambiguate emotionally ambiguous scenarios and in no other training conditions. This may limit the extent of the effect as it suggests that a very specific strategy has been trained rather than the modification of an interpretive bias that may transfer to other tasks. While recent meta-analyses reveal that CBM-I training has a relatively strong effect on interpretation biases $g=0.81$, Hallion \& Ruscio, 2011), there was large variation between the types of tasks included for analysis. This means that tasks that may not reflect a true change in interpretive bias (e.g., delayed tasks) were included in the analysis. A recent review of CBM-I has highlighted a low number of quality studies, with small $\mathrm{N}$ and possible evidence of publication bias, highlighting the need for a structured approach to determining the critical factors involved in modifying an interpretive bias and defining the extent of training effects (Cristea, Kok \& Cuijpers, 2015). Therefore in order to ascertain the impact of training on true interpretive change, further research must take place examining the generalisation of interpretive biases to other tasks and measures of moods to ensure the validity of the effect.

Finally, I did not include a no-training control condition within this set of studies in order to determine a baseline against which negative and benign training effects could be compared. It is important to look further into the discrepant effects of negative and benign training effects, as it is not certain that these effects work by the same mechanisms or have completely opposing effects on interpretive biases to one another. Negative valence is a better constrained 
emotional category, and possibly better suited for researching the impact of training on psycholinguistic variables than benign valence, which is a mixture of positive and neutral interpretations. However, benign (or even positive) training may be more clinically relevant, and may better reveal the role of alleviated biases on mood and emotional reactivity. Whilst it is possible to consider Experiment 3 as a possible control condition, as participants simply read unambiguous scenarios, a true no-training baseline would establish a point from which other research into underlying negative and benign processing biases could be examined.

\section{Conclusions}

In a series of studies, the near-transfer effects of CBM-I training were investigated. Traditionally, little consideration has been given to the types of methodology used during CBM-I training or test phases. As a result, previous studies have been limited in their ability to inform our understanding of the conditions under which an interpretive bias is likely to emerge, or to establish the mechanism(s) that might subserve the induction of an emotional interpretation bias. Instead, research has tended to focus on the relationship between emotional interpretive biases and measures of emotional reactivity, emotional regulation or mood state. Overall, the results of the studies within this thesis suggest that a genuine change in interpretive processing takes place following a single application of the novel scenario-based training used in this thesis. However, a change in interpretive bias is dependent on ambiguity resolution in the training phase, only appears at a later selection stage of meaning resolution and is dependent on semantic evaluation of the relationship between the prime and the target.

In conclusion, the results of this thesis highlight the need for further research in the area of interpretive bias and the importance of considering the ways in which applied clinical and psycholinguistic research domains can inform one another. It is important to consider the role of interpretation beyond linguistic situations, as interpretation plays a role in our understanding of many emotionally ambiguous situations including our processing of facial expressions, tone of voice and the actions and intentions of other people. The interpretation of emotional ambiguity is an efficient and relatively unconscious process that 
informs our understanding of the world and determines our later actions and interactions with others. As a result interpretations contribute greatly to our understanding of the world and have implications for our future behaviour and interactions. There is still a lot more research to be done on interpretive processes, their dysfunction in clinical disorders and the implications for how we navigate and understand the world around us. 
Appendix A

Training Scenarios for Experiment 1a, 1b, 2 and Experiment 5.

\begin{tabular}{|c|c|c|}
\hline Ambiguous Scenario & $\begin{array}{l}\text { Negative } \\
\text { Word Fragment }\end{array}$ & $\begin{array}{l}\text { Completed } \\
\text { Fragment }\end{array}$ \\
\hline You need to take some time off for the ceremony. & F_ne_al & Funeral \\
\hline Your mother calls to tell you the news. & D_a_h & Death \\
\hline You are going to a party where you don't know anyone. & Scar_d & Scared \\
\hline Your phone rings early in the morning. & Ac_id_nt & Accident \\
\hline $\begin{array}{l}\text { You are walking down an alleyway when you notice } \\
\text { something. }\end{array}$ & Ro_b_r & Robber \\
\hline $\begin{array}{l}\text { You try on some clothing but your friend advises you not } \\
\text { to buy it. }\end{array}$ & U_ly & Ugly \\
\hline $\begin{array}{l}\text { You hear your friends discuss a party that you were } \\
\text { unaware of. }\end{array}$ & E_cl_ded & Excluded \\
\hline You notice your friend's face as she opens your present. & Dis_ppoi_ted & Disappointed \\
\hline You are thinking about your new job. & An_i_us & Anxious \\
\hline $\begin{array}{l}\text { You are talking to an acquaintance who keeps looking out } \\
\text { the window. }\end{array}$ & B_ri_g & Boring \\
\hline A noise in the middle of the night wakes you up. & B_r_lar & Burglar \\
\hline The test result reflects your abilities. & F_il & Fail \\
\hline Your mother never expected you to become who you are. & Pr_so_ & Prison \\
\hline You've got an unusual passion. & Fr_ak & Freak \\
\hline The dog is running towards you. & At_a_k & Attack \\
\hline You can feel the sweat running down your spine. & T_r_or & Terror \\
\hline You notice a man wearing dark glasses. & Su_pic_ous & Suspicious \\
\hline You notice the car is going a lot faster than the bike. & D_nge_ous & Dangerous \\
\hline Your coffee is really hot. & Bu_n & Burn \\
\hline Suddenly the lights go out and you hear voices. & In_r_der & Intruder \\
\hline Your lawyer registers the value of your property. & D_vo_ce & Divorce \\
\hline No-one can finish the dinner you made. & Dis_ust_ng & Disgusting \\
\hline The boss wants to talk to you. & Fir_d & Fired \\
\hline When you reach the bus stop, people are laughing. & Los_r & Loser \\
\hline Everyone is quiet during your presentation. & A_le_p & Asleep \\
\hline Your child can't walk & D_sab_ed & Disabled \\
\hline People laugh as you deliver your speech & S_up_d & Stupid \\
\hline Everyone is looking at you. & Em_arr_ssed & Embarrassed \\
\hline Not many of your friends are around these days. & Re_ect_d & Rejected \\
\hline Your puppy is lying very still. & D_ad & Dead \\
\hline You're usually single. & Un_tt_active & Unattractive \\
\hline Your flat is very cheap to rent. & D_r_y & Dirty \\
\hline The class gives you feedback about your speech. & Ne_at_ve & Negative \\
\hline Your sister is now bald. & C_n_er & Cancer \\
\hline You've got nothing to do today. & Bo_ed & Bored \\
\hline Your flatmate yells for you to come downstairs. & Tr_u_le & Trouble \\
\hline You can't accept the job offer. & Il_n_ss & Illness \\
\hline You may as well give away the baby clothes now. & Misc_rria_e & Miscarriage \\
\hline You notice something in the stranger's hand. & W_ap_n & Weapon \\
\hline Your partner has something to tell you & Du_p_d & Dumped \\
\hline You don't have many friends here. & Al_ne & Alone \\
\hline You take a deep breath and jump. & Su_ci_e & Suicide \\
\hline $\begin{array}{l}\text { You're very different to anyone your partner has ever } \\
\text { dated. }\end{array}$ & W_r_e & Worse \\
\hline
\end{tabular}


You feel something brushing your arm.

The smell hits you as soon as you walk into the room.
S_id_r

Un_le_sant
Spider

Unpleasant 


\begin{tabular}{|c|c|c|}
\hline Ambiguous Scenario & $\begin{array}{l}\text { Benign } \\
\text { Word Fragment }\end{array}$ & $\begin{array}{l}\text { Completed } \\
\text { Fragment }\end{array}$ \\
\hline You need to take some time off for the ceremony. & Gr_dua_ion & Graduation \\
\hline Your mother calls to tell you the news. & Mar_i_ge & Marriage \\
\hline You are going to a party where you don't know anyone. & ex_it_d & Excited \\
\hline Your phone rings early in the morning. & Bi_th & Birth \\
\hline You are walking down an alleyway when you notice & $\mathrm{C}_{-} \mathrm{t}$ & Cat \\
\hline $\begin{array}{l}\text { You try on some clothing but your friend advises you not to } \\
\text { buy it. } \\
\text { You hear your friends discuss a party that you were }\end{array}$ & E_pens_ve & Expensive \\
\hline unaware of. & in_it_d & Invited \\
\hline You notice your friend's face as she opens your present. & del_ght_d & Delighted \\
\hline You are thinking about your new job. & p_eas_d & Pleased \\
\hline $\begin{array}{l}\text { You are talking to an acquaintance who keeps looking out } \\
\text { the window. }\end{array}$ & di_trac_ed & Distracted \\
\hline A noise in the middle of the night wakes you up. & D_g & Dog \\
\hline The test result reflects your abilities. & sm_rt & Smart \\
\hline Your mother never expected you to become who you are. & pr_ud & Proud \\
\hline You've got an unusual passion. & In_er_sting & Interesting \\
\hline The dog is running towards you. & pl_y & Play \\
\hline You can feel the sweat running down your spine. & S_m_er & Summer \\
\hline You notice a man wearing dark glasses. & su_sh_ne & Sunshine \\
\hline You notice the car is going a lot faster than the bike. & ove_t_ke & Overtake \\
\hline Your coffee is really hot. & De_ic_ous & Delicious \\
\hline Suddenly the lights go out and you hear voices. & Mo_ie & Movie \\
\hline Your lawyer registers the value of your property. & Ins_ra_ce & Insurance \\
\hline No-one can finish the dinner you made. & $\mathrm{Fu}_{\mathrm{l}} \mathrm{l}$ & Full \\
\hline The boss wants to talk to you. & pr_mo_ed & Promoted \\
\hline When you reach the bus stop, people are laughing. & f_nny & Friendly \\
\hline Everyone is quiet during your presentation. & at_ent_ve & Attentive \\
\hline Your child can't walk & i_f_nt & Infant \\
\hline People laugh as you deliver your speech & $f_{-} n_{-} \mathrm{y}$ & Funny \\
\hline Everyone is looking at you. & po_ul_r & Popular \\
\hline Not many of your friends are around these days. & ove_se_s & Overseas \\
\hline Your puppy is lying very still. & as_eep & Asleep \\
\hline You're usually single. & Ca_efr_e & Carefree \\
\hline Your flat is very cheap to rent. & ba_g_in & Bargain \\
\hline The class gives you feedback about your speech. & Po_it_ve & Positive \\
\hline Your sister is now bald. & ecce_tr_c & Eccentric \\
\hline You've got nothing to do today. & rel_x & Relax \\
\hline Your flatmate yells for you to come downstairs. & so_iabl_ & Sociable \\
\hline You can't accept the job offer. & com_itt_d & Committed \\
\hline You may as well give away the baby clothes now. & Gr_wn & Grown \\
\hline You notice something in the stranger's hand. & p_one & Phone \\
\hline Your partner has something to tell you & L_ve & Love \\
\hline You don't have many friends here. & F_rei_n & Foreign \\
\hline You take a deep breath and jump. & swi_mi_g & Swimming \\
\hline You're very different to anyone your partner has ever dated. & sp_c_al & Special \\
\hline You feel something brushing your arm. & Gra_s & Grass \\
\hline The smell hits you as soon as you walk into the room. & ince_s_ & Incense \\
\hline
\end{tabular}


Appendix B

Training Scenarios for Experiment 4

\begin{tabular}{l} 
Ambiguous Scenario \\
\hline You need to take some time off for the ceremony.
\end{tabular}

Your mother calls to tell you the news.

You are going to a party where you don't know anyone.

Your phone rings early in the morning.

You are walking down an alleyway when you notice something.

You try on some clothing but your friend advises you not to buy it.

You hear your friends discuss a party that you were unaware of.

You notice your friend's face as she opens your present.

You are thinking about your new job.

You are talking to an acquaintance who keeps looking out the window.

A noise in the middle of the night wakes you up.

The test result reflects your abilities.

Your mother never expected you to become who you are.

You've got an unusual passion.

The dog is running towards you.

You can feel the sweat running down your spine.

You notice a man wearing dark glasses.

You notice the car is going a lot faster than the bike.

Your coffee is really hot.

Suddenly the lights go out and you hear voices.

Your lawyer registers the value of your property.

No-one can finish the dinner you made.

The boss wants to talk to you.

When you reach the bus stop, people are laughing. Everyone is quiet during your presentation.

Your child can't walk.

People laugh as you deliver your speech.

Everyone is looking at you.

Not many of your friends are around these days.

Your puppy is lying very still.

You're usually single.

Your flat is very cheap to rent.

The class gives you feedback about your speech.

Your sister is now bald.

You've got nothing to do today.

Your flatmate yells for you to come downstairs.

You can't accept the job offer.

You may as well give away the baby clothes now.
New Negative Scenario

Your dead grandmother's service is today

Your upset mother calls to tell you the Death news

You are not looking forward to the party

Your phone call is unexpected

You hear a shout as you are walking

down an alleyway

Your friend tells you the dress is too ugly

You don't receive a party notification on facebook

Your upset friend dislikes her birthday present

You haven't been offered a new job

Your friend isn't listening to you talk

A stranger enters your house

You are unhappy with your test results

Your unsupportive peers judge your

actions

Your unhealthy hobby is absorbing

The angry dog is running towards you

Sitting in anticipation makes you sweat

A dodgy man is standing next to you

The car behind you is driving too slowly

You hate hot coffee

You are terrified as the lights go out in your house

You are forced to value all your

posessions

Your upset guests can't finish the meal you made

Your angry boss wants to chat to you

Your friends ignore you at the bus stop

The bored people listen to your talk

Your sick child hasn't started walking

People laugh meanly as you deliver your speech

Your clumsiness makes everyone laugh

You don't receive postcards from your friends

Your sick puppy is lying very still

Your date stands you up

Your gross flat is very cheap to rent

Your disappointed teacher gives you feedback about the test

Your sick sister has shaved her head

You are sad you have nothing on today

Your mad flatmate yells at you to come downstairs

You are too sick to accept the job offer

Your wife lost the baby
Word Fragment

Funeral

Scared

Accident

Robber

Ugly

Excluded

Disappointed

Anxious

Boring

Burglar

Fail

Prison

Freak

Attack

Terror

Suspicious

Dangerous

Burn

Intruder

Divorce

Disgusting

Fired

Loser

Asleep

Disabled

Stupid

Embarrassed

Rejected

Dead

Unattractive

Dirty

Negative

Cancer

Bored

Trouble

Illness

Miscarriage 
You notice something in the stranger's hand.

Your partner has something to tell you.

You don't have many friends here.

You take a deep breath and jump.

You're very different to anyone your partner has ever dated.

You feel something brushing your arm.

The smell hits you as soon as you walk into the room.
The armed stranger confronts you

Weapon

Your angry partner has something to tell you

As an outsider you haven't made new friends yet

You jump off the bridge

Your upset partner comments on your individuality

You brush cobwebs off your arm

The smell of decay hits you when you walk into the room
Dumped

Alone

Suicide

Worse

Spider

Unpleasant 


\begin{tabular}{|c|c|c|}
\hline Ambiguous Scenario & New Benign Scenario & Word Fragment \\
\hline You need to take some time off for the ceremony. & Your brother's degree celebration is today & Graduation \\
\hline Your mother calls to tell you the news. & $\begin{array}{l}\text { Your excited mother calls to tell you the } \\
\text { news }\end{array}$ & Marriage \\
\hline $\begin{array}{l}\text { You are going to a party where you don't know } \\
\text { anyone. }\end{array}$ & You are looking forward to the party & Excited \\
\hline Your phone rings early in the morning. & Your wakeup call is expected & Birth \\
\hline $\begin{array}{l}\text { You are walking down an alleyway when you } \\
\text { notice something. }\end{array}$ & $\begin{array}{l}\text { You hear a meow as you are walking down } \\
\text { an alleyway }\end{array}$ & Cat \\
\hline $\begin{array}{l}\text { You try on some clothing but your friend advises } \\
\text { you not to buy it. }\end{array}$ & Your friend tells you the dress is too much & Expensive \\
\hline $\begin{array}{l}\text { You hear your friends discuss a party that you } \\
\text { were unaware of. }\end{array}$ & $\begin{array}{l}\text { You receive a party notification on } \\
\text { facebook }\end{array}$ & Invited \\
\hline $\begin{array}{l}\text { You notice your friend's face as she opens your } \\
\text { present. }\end{array}$ & $\begin{array}{l}\text { Your delighted friend loves her birthday } \\
\text { present }\end{array}$ & Delighted \\
\hline You are thinking about your new job. & You have been offered a new job & Pleased \\
\hline $\begin{array}{l}\text { You are talking to an acquaintance who keeps } \\
\text { looking out the window. }\end{array}$ & Your friend is listening to you talk & Distracted \\
\hline A noise in the middle of the night wakes you up. & Your dog enters your house & Dog \\
\hline The test result reflects your abilities. & You are pleased with your test results & Smart \\
\hline $\begin{array}{l}\text { Your mother never expected you to become who } \\
\text { you are. }\end{array}$ & Your supportive peers judge your actions & Proud \\
\hline You've got an unusual passion. & Your exciting hobby is absorbing & Interesting \\
\hline The dog is running towards you. & The friendly dog is running towards you & Play \\
\hline You can feel the sweat running down your spine. & Sitting in the sun makes you sweat & Summer \\
\hline You notice a man wearing dark glasses. & A sunburnt man is standing next to you & Sunshine \\
\hline $\begin{array}{l}\text { You notice the car is going a lot faster than the } \\
\text { bike. }\end{array}$ & The car in front of you is driving slowly & Overtake \\
\hline Your coffee is really hot. & You love hot coffee & Delicious \\
\hline Suddenly the lights go out and you hear voices. & $\begin{array}{l}\text { You are excited as the lights go out in the } \\
\text { movie theatre }\end{array}$ & Movie \\
\hline Your lawyer registers the value of your property. & You want to value all your posessions & Insurance \\
\hline No-one can finish the dinner you made. & $\begin{array}{l}\text { Your happy guests can't finish the meal } \\
\text { you made }\end{array}$ & Full \\
\hline The boss wants to talk to you. & Your friendly boss wants to chat to you & Promoted \\
\hline When you reach the bus stop, people are laughing. & $\begin{array}{l}\text { Your friends are smiling at you at the bus } \\
\text { stop }\end{array}$ & Friendly \\
\hline Everyone is quiet during your presentation. & The absorbed people listen to your talk & Attentive \\
\hline Your child can't walk. & Your young chid hasn't started walking & Infant \\
\hline People laugh as you deliver your speech. & $\begin{array}{l}\text { People laugh appreciatively as you deliver } \\
\text { your speech }\end{array}$ & Funny \\
\hline Everyone is looking at you. & Your jokes make everyone laugh & Popular \\
\hline Not many of your friends are around these days. & You receive postcards from your friends & Overseas \\
\hline Your puppy is lying very still. & Your exhausted puppy is lying very still & Asleep \\
\hline $\begin{array}{l}\text { You're usually single. } \\
\text { Your flat is very cheap to rent. }\end{array}$ & $\begin{array}{l}\text { You enjoy being single } \\
\text { Your beautiful flat is very cheap to rent }\end{array}$ & $\begin{array}{l}\text { Carefree } \\
\text { Bargain }\end{array}$ \\
\hline The class gives you feedback about your speech. & $\begin{array}{l}\text { Your proud teacher dives you feedback } \\
\text { about the test }\end{array}$ & Positive \\
\hline Your sister is now bald. & Your unique sister has shaved her head & Eccentric \\
\hline You've got nothing to do today. & $\begin{array}{l}\text { You are happy that you have nothing on } \\
\text { today }\end{array}$ & Relax \\
\hline Your flatmate yells for you to come downstairs. & $\begin{array}{l}\text { Your excited flatmate yells at you to come } \\
\text { downstairs }\end{array}$ & Sociable \\
\hline You can't accept the job offer. & $\begin{array}{l}\text { You are too in demand to accept the job } \\
\text { offer }\end{array}$ & Committed \\
\hline $\begin{array}{l}\text { You may as well give away the baby clothes now. } \\
\text { their baby clothes }\end{array}$ & Your child no longer fits & Grown \\
\hline You notice something in the stranger's hand. & The lost stranger confronts you & Phone \\
\hline
\end{tabular}


Your partner has something to tell you.

You don't have many friends here.

You take a deep breath and jump.

You're very different to anyone your partner has ever dated.

You feel something brushing your arm.

The smell hits you as soon as you walk into the room.
Your adoring partner has something to tell Love you

As an immigrant you haven't made new friends yet

You dive off the bridge

Foreign

Your caring partner comments on your individuality

You brush clippings off your arm Grass

The smell of lavender hits you when you Incense walk into the room
Swimming

Special 
Appendix C

Prime and Target words for Experiment 4

\begin{tabular}{|c|c|c|c|c|c|c|c|}
\hline $\begin{array}{l}\text { Prime - } \\
\text { List A }\end{array}$ & Target & Valence & RelType & $\begin{array}{l}\text { Prime- } \\
\text { List B }\end{array}$ & Target & Valence & Reltype \\
\hline arms & weapons & Negative & Related & arms & legs & Neutral & Related \\
\hline beat & bash & Negative & Related & beat & rhythm & Neutral & Related \\
\hline bit & insect & Negative & Related & bit & part & Neutral & Related \\
\hline block & obstruct & Negative & Related & block & apartment & Neutral & Related \\
\hline box & fight & Negative & Related & box & gift & Neutral & Related \\
\hline break & smash & Negative & Related & break & lunch & Neutral & Related \\
\hline club & assault & Negative & Related & club & group & Neutral & Related \\
\hline crush & destroy & Negative & Related & crush & like & Neutral & Related \\
\hline desert & abandon & Negative & Related & desert & sand & Neutral & Related \\
\hline escape & flee & Negative & Related & escape & holiday & Neutral & Related \\
\hline fall & over & Negative & Related & fall & autumn & Neutral & Related \\
\hline fell & down & Negative & Related & fell & trees & Neutral & Related \\
\hline fine & penalty & Negative & Related & fine & happy & Neutral & Related \\
\hline flounder & drown & Negative & Related & flounder & fish & Neutral & Related \\
\hline frame & crime & Negative & Related & frame & picture & Neutral & Related \\
\hline glare & angy & Negative & Related & glare & sun & Neutral & Related \\
\hline grill & interrogate & Negative & Related & grill & barbeque & Neutral & Related \\
\hline hamper & stop & Negative & Related & hamper & picnic & Neutral & Related \\
\hline hard & difficult & Negative & Related & hard & concrete & Neutral & Related \\
\hline hiding & whipping & Negative & Related & hiding & game & Neutral & Related \\
\hline hit & whack & Negative & Related & hit & successful & Neutral & Related \\
\hline issue & difficulty & Negative & Related & issue & magazine & Neutral & Related \\
\hline jerk & idiot & Negative & Related & jerk & tug & Neutral & Related \\
\hline lash & whips & Negative & Related & lash & eye & Neutral & Related \\
\hline leaves & goes & Negative & Related & leaves & plant & Neutral & Related \\
\hline left & deserted & Negative & Related & left & right & Neutral & Related \\
\hline mean & average & Neutral & Related & mean & cruel & Negative & Related \\
\hline miss & lady & Neutral & Related & miss & lonely & Negative & Related \\
\hline parting & hair & Neutral & Related & parting & goodbye & Negative & Related \\
\hline patient & tolerant & Neutral & Related & patient & sick & Negative & Related \\
\hline poach & egg & Neutral & Related & poach & steal & Negative & Related \\
\hline punch & drink & Neutral & Related & punch & fist & Negative & Related \\
\hline quack & duck & Neutral & Related & quack & doctor & Negative & Related \\
\hline ram & sheep & Neutral & Related & ram & shoulder & Negative & Related \\
\hline sack & potatoes & Neutral & Related & sack & job & Negative & Related \\
\hline shady & sunny & Neutral & Related & shady & dodgy & Negative & Related \\
\hline slip & petticoat & Neutral & Related & slip & stagger & Negative & Related \\
\hline squash & vegetable & Neutral & Related & squash & flat & Negative & Related \\
\hline stalk & flower & Neutral & Related & stalk & creep & Negative & Related \\
\hline stole & fur & Neutral & Related & stole & thief & Negative & Related \\
\hline strain & noodles & Neutral & Related & strain & stress & Negative & Related \\
\hline strained & sieve & Neutral & Related & strained & tired & Negative & Related \\
\hline strike & bowling & Neutral & Related & strike & slap & Negative & Related \\
\hline striking & attractive & Neutral & Related & striking & beating & Negative & Related \\
\hline stub & ticket & Neutral & Related & stub & hurt & Negative & Related \\
\hline tackle & bait & Neutral & Related & tackle & grab & Negative & Related \\
\hline tank & water & Neutral & Related & tank & war & Negative & Related \\
\hline tart & pie & Neutral & Related & tart & slut & Negative & Related \\
\hline terminal & airport & Neutral & Related & terminal & dying & Negative & Related \\
\hline trip & vacation & Neutral & Related & trip & stumble & Negative & Related \\
\hline vice & tool & Neutral & Related & vice & $\sin$ & Negative & Related \\
\hline
\end{tabular}




\begin{tabular}{|c|c|c|c|c|c|c|c|}
\hline will & urge & Neutral & Related & will & inheritance & Negative & Related \\
\hline arms & trousers & Neutral & Unrelated & arms & greedy & Negative & Unrelated \\
\hline beat & vest & Neutral & Unrelated & beat & liar & Negative & Unrelated \\
\hline bit & swing & Neutral & Unrelated & bit & phony & Negative & Unrelated \\
\hline block & fly & Neutral & Unrelated & block & hateful & Negative & Unrelated \\
\hline box & poster & Neutral & Unrelated & box & jealous & Negative & Unrelated \\
\hline break & paint & Neutral & Unrelated & break & coffin & Negative & Unrelated \\
\hline club & windmill & Neutral & Unrelated & club & deaf & Negative & Unrelated \\
\hline crush & kettle & Neutral & Unrelated & crush & nasty & Negative & Unrelated \\
\hline desert & bacon & Neutral & Unrelated & desert & bitter & Negative & Unrelated \\
\hline escape & hammer & Neutral & Unrelated & escape & scold & Negative & Unrelated \\
\hline fall & boat & Neutral & Unrelated & fall & owe & Negative & Unrelated \\
\hline fell & brush & Neutral & Unrelated & fell & dirt & Negative & Unrelated \\
\hline fine & lawn & Neutral & Unrelated & fine & noose & Negative & Unrelated \\
\hline flounder & cup & Neutral & Unrelated & flounder & disease & Negative & Unrelated \\
\hline frame & hairpin & Neutral & Unrelated & frame & dislike & Negative & Unrelated \\
\hline glare & beverage & Neutral & Unrelated & glare & anorexic & Negative & Unrelated \\
\hline grill & folder & Neutral & Unrelated & grill & dull & Negative & Unrelated \\
\hline hamper & diary & Neutral & Unrelated & hamper & fake & Negative & Unrelated \\
\hline hard & elephant & Neutral & Unrelated & hard & smelly & Negative & Unrelated \\
\hline hiding & driver & Neutral & Unrelated & hiding & toxic & Negative & Unrelated \\
\hline hit & jacket & Neutral & Unrelated & hit & vomit & Negative & Unrelated \\
\hline issue & leg & Neutral & Unrelated & issue & squeeze & Negative & Unrelated \\
\hline jerk & fork & Neutral & Unrelated & jerk & bomb & Negative & Unrelated \\
\hline lash & knee & Neutral & Unrelated & lash & fearful & Negative & Unrelated \\
\hline leaves & lock & Neutral & Unrelated & leaves & germs & Negative & Unrelated \\
\hline left & tall & Neutral & Unrelated & left & dumb & Negative & Unrelated \\
\hline mean & crash & Negative & Unrelated & mean & pebble & Neutral & Unrelated \\
\hline miss & zombie & Negative & Unrelated & miss & rubber & Neutral & Unrelated \\
\hline parting & garbage & Negative & Unrelated & parting & silk & Neutral & Unrelated \\
\hline patient & bullet & Negative & Unrelated & patient & shoe & Neutral & Unrelated \\
\hline poach & lame & Negative & Unrelated & poach & lion & Neutral & Unrelated \\
\hline punch & coward & Negative & Unrelated & punch & razor & Neutral & Unrelated \\
\hline quack & weep & Negative & Unrelated & quack & nun & Neutral & Unrelated \\
\hline ram & scary & Negative & Unrelated & ram & statue & Neutral & Unrelated \\
\hline sack & argue & Negative & Unrelated & sack & lottery & Neutral & Unrelated \\
\hline shady & offensive & Negative & Unrelated & shady & pig & Neutral & Unrelated \\
\hline slip & weasel & Negative & Unrelated & slip & curtains & Neutral & Unrelated \\
\hline squash & immature & Negative & Unrelated & squash & clock & Neutral & Unrelated \\
\hline stalk & scar & Negative & Unrelated & stalk & mountain & Neutral & Unrelated \\
\hline stole & gossip & Negative & Unrelated & stole & mail & Neutral & Unrelated \\
\hline strain & unfair & Negative & Unrelated & strain & palace & Neutral & Unrelated \\
\hline strained & evil & Negative & Unrelated & strained & passage & Neutral & Unrelated \\
\hline strike & desperate & Negative & Unrelated & strike & frog & Neutral & Unrelated \\
\hline striking & traitor & Negative & Unrelated & striking & farm & Neutral & Unrelated \\
\hline stub & wasp & Negative & Unrelated & stub & jug & Neutral & Unrelated \\
\hline tackle & cemetery & Negative & Unrelated & tackle & journal & Neutral & Unrelated \\
\hline $\operatorname{tank}$ & snippy & Negative & Unrelated & $\operatorname{tank}$ & hat & Neutral & Unrelated \\
\hline tart & stink & Negative & Unrelated & tart & key & Neutral & Unrelated \\
\hline terminal & snob & Negative & Unrelated & terminal & elbow & Neutral & Unrelated \\
\hline trip & tomb & Negative & Unrelated & trip & lamp & Neutral & Unrelated \\
\hline vice & silly & Negative & Unrelated & vice & doll & Neutral & Unrelated \\
\hline will & abuse & Negative & Unrelated & will & smooth & Neutral & Unrelated \\
\hline
\end{tabular}




\section{References}

Amir, N., \& Taylor, C. T. (2012). Interpretation training in individuals with generalized social anxiety disorder: A randomized controlled trial. Journal of Consulting and Clinical Psychology, 80(3), 497-511. doi:10.1037/a0026928

Balota, D. a, \& Paul, S. T. (1996). Summation of activation: evidence from multiple primes that converge and diverge within semantic memory. Journal of Experimental Psychology. Learning, Memory, and Cognition, 22(4), 827-845. doi:10.1037/0278-7393.22.4.827

Balota, D. A., Yap, M. J., \& Cortese, M. J. (2006). Visual word recognition: The journey from features to meaning (a travel update). In M. Traxler \& M. A. Gernsbacher (Eds.), Handbook of psycholinguistics (pp. 285-375). Amsterdam: Academic Press.

Balota, D. A. (1994). Visual word recognition: The journey from features to meaning. In M. A. Gernsbacher (Ed.), Handbook of psycholinguistics (pp. 303-348). San Diego, CA: Academic Press.

Balota, D. A., \& Chumbley, J. I. (1984). Are lexical decisions a good measure of lexical access? The role of word frequency in the neglected decision stage. Journal of Experimental Psychology: Human Perception \& Performance, 10, 340-357.

Barsalou, L. W. (1999). Perceptual symbol systems. The Behavioral and Brain Sciences, 22(4), 577-609; discussion 610-660. doi:10.1017/S0140525X99252144

Beard, C. (2011). Cognitive bias modification for anxiety: Current evidence and future directions. Expert Review of Neurotherapeutics, 11, 299-311. http://dx.doi.org/10.1586/ern.10.194

Beard, C., \& Amir, N. (2008). A multi-session interpretation modification program: Changes in interpretation and social anxiety symptoms. Behaviour Research and Therapy, 46(10), 1135-1141. doi:10.1016/j.brat.2008.05.012

Beck, A. T. (1991). A 30- Year Retrospective. American Psychologist, 46(4), 368375. http://dx.doi.org/10.1037/0003-066X.46.4.368

Beck, A. T. (1967). Depression: Clinical, experimental, and theoretical aspects. New York, NY: Harper \& Row.

Becker, S., Moscovitch, M., Behrmann, M., \& Joordens, S. (1997). Long-term semantic priming: a computational account and empirical evidence. Journal of Experimental Psychology. Learning, Memory, and Cognition, 23(5), 10591082. doi:10.1037/0278-7393.23.5.1059 
Binder, K. S., \& Morris, R. K. (1995). Eye movements and lexical ambiguity resolution: effects of prior encounter and discourse topic. Journal of Experimental Psychology. Learning, Memory, and Cognition, 21(5), 11861196. doi:10.1037/0278-7393.21.5.1186

Blanchette, I., \& Richards, A. (2010). The influence of affect on higher level cognition: A review of research on interpretation, judgement, decision making and reasoning. Cognition and Emotion, 24(4), 37-41. doi:10.1080/02699930903132496

Bower, G. H. (1981). Mood and memory. The American Psychologist, 36(2), 129148. doi:10.1037/0003-066X.36.2.129

Bowler, J. O., Mackintosh, B., Dunn, B. D., Mathews, A., Dalgleish, T., \& Hoppitt, L. (2012). A Comparison of Cognitive Bias Modification for Interpretation and Computerized Cognitive Behavior Therapy: Effects on Anxiety, Depression, Attentional Control, and Interpretive Bias. Journal of Consulting and Clinical Psychology, 80(6), 1021-1033. doi:10.1037/a0029932

Bradley, M.M., \& Lang, P.J. (1999). Affective norms for English words (ANEW): Instruction manual and affective ratings. Technical Report $\mathrm{C}-1$, The Center for Research in Psychophysiology, University of Florida.

Bradley, B. P., Mogg, K., \& Williams, R. (1994). Implicit and explicit memory for emotional information in non-clinical subjects. Behaviour Research and Therapy, 32(1), 65-78. doi:10.1016/0005-7967(94)90085-X

Budiu, R., \& Anderson, J. R. (2004). Interpretation-based processing: A unified theory of semantic sentence comprehension. Cognitive Science, 28(1), 1-44. doi:10.1016/j.cogsci.2003.10.001

Butler, G., \& Mathews, A. (1983). Cognitive processes in anxiety. Advances in Behaviour Research and Therapy, 5(1), 51-62. doi:10.1016/01466402(83)90015-2

Caramazza, A. (1997). How Many Levels of Processing Are There in Lexical Access? Cognitive Neuropsychology, 14(1), 177-208. doi:10.1080/026432997381664

Carretié, L., Martín-Loeches, M., Hinojosa, J. A, \& Mercado, F. (2001). Emotion and attention interaction studied through event-related potentials. Journal of Cognitive Neuroscience, 13(8), 1109-1128. doi:10.1162/089892901753294400

Carroll, J. B., \& White, M. N. (1973). Word frequency and age of acquisition as determiners of picture-naming latency. Quarterly Journal of Experimental Psychology, 25(1), 85-95. doi:10.1080/14640747308400325 
Challis, B. H., \& Krane, R. V. (1988). Mood induction and the priming of semantic memory in a lexical decision task: Asymmetric effects of elation and depression. Bulletin of the Psychonomic Society, 26(4), 309-312. doi:10.3758/BF03337666

Chwilla, D. J., \& Kolk, H. H. J. (2003). Event-related potential and reaction time evidence for inhibition between alternative meanings of ambiguous words. Brain and Language, 86(2), 167-192. doi:10.1016/S0093-934X(02)00527-8

Clark, D. M. \& Wells, A. (1995). A cognitive model of social phobia. In R.G. Heimberg, M.R. Liebowitz, D. A. Hope and F. R. Schneier (Eds.), Social Phobia: diagnosis, assessment, and treatment (pp. 69-91). New York: Guilford Press

Clarke P. J. F., Nanthakumar S., Notebaert L., Holmes E. A., Blackwell S. E., \& MacLeod C. (2014). Simply imagining sunshine, lollipops and rainbows will not budge the bias: The role of ambiguity in interpretive bias modification. Cognitive Therapy and Research, 38, 120131. doi:10.1007/s10608-013-9564-X

Collins, A. M., \& Loftus, E. F. (1975). A spreading-activation theory of semantic processing. Psychological Review, 82(6), 407-428. doi:10.1037/0033295X.82.6.407

Clark, D. M., Teasdale, J. D., Broadbent, D. E., \& Martin, M. (1983). Effect of mood on lexical decisions. Bulletin of the Psychonomic Society, 21, 175-178.

Cristea, I. A., Kok, R. N., \& Cujipers, P. (2015). The efficacy of cognitive bias modification interentions in anxiety and depression: A meta-analysis. British Journal of Psychiatry, 206, 7-16. doi:10.1192/bjp.bp.114.146761

Coulson S, \& Federmeier K., D. (In Press). Words in context: ERPs and the lexical/postlexical distinction. Journal of Psycholinguistic Research

De Groot, A. M. B. (1984). Primed lexical decision: Combined effects of the proportion of related prime-target pairs and the stimulus-onset asynchrony of prime and target. Quarterly Journal of Experimental Psychology, 36A, 253280.

De Houwer, J., Hermans, D., \& Eelen, P. (1998). Affective and Identity Priming with Episodically Associated Stimuli. Cognition \& Emotion, 12(2), 145-169. doi:10.1080/026999398379691

Draine, S. C., \& Greenwald, A. G. (1998). Replicable unconscious semantic priming. Journal of Experimental Psychology. General, 127(3), 286-303. doi:10.1037/0096-3445.127.3.286

Duffy, S. A, Morris, R. K., \& Rayner, K. (1988). Lexical ambiguity and fixation time in reading. Journal of Memory and Language, 27, 429-446. 
Eysenck, M. W., Mogg, K., May, J., Richards, A., \& Mathews, A. (1991). Bias in interpretation of ambiguous sentences related to threat in anxiety. Journal of Abnormal Psychology, 100, 144-50.

Fazio, R. H., Sanbonmatsu, D. M., Powell, M. C., \& Kardes, F. R. (1986). On the automatic activation of attitudes. Journal of Personality and Social Psychology, 50(2), 229-238. doi:10.1037/0022-3514.50.2.229

Forster, K. I. (1981). Priming and the effects of sentence and lexical contexts on naming time: Evidence for autonomous lexical processing. The Quarterly Journal of Experimental Psychology Section A, 33(4), 465-495. doi:10.1080/14640748108400804

Frauenfelder, U. H., \& Tyler, L. K. (1987). The process of spoken word recognition: an introduction. Cognition, 25(1-2), 1-20. doi:10.1016/00100277(87)90002-3

Gawlick-Grendell, L. A., \& Woltz, D. J. (1994). Meaning dominance norms for 120 homographs. Behavior Research Methods, Instruments, \& Computers, 26, 525.

Gee, N. R., \& Harris, S. L. (2010). Homograph norms: an alternative approach to determining meaning dominance. Behavior Research Methods, 42(4), 976986. doi:10.3758/BRM.42.4.976

Gernsbacher, M. A., Varner, K. R., \& Faust, M. E. (1990). Investigating differences in general comprehension skill. Journal of Experimental Psychology. Learning, Memory, and Cognition, 16(3), 430-445. doi:10.1037/02787393.16.3.430

Gernsbacher, M.A., St. John, M.F., (2001). Modeling suppression in lexical access.

In D. S. Gorfein (Ed.), On the Consequences of Meaning Selection: Perspectives on Resolving Lexical Ambiguity.(pp. 47-65). Washington, DC: American Psychological Association.

Gorfein, D. S., \& Bubka, A. (1989). A context sensitive frequency based theory of meaning achievement. In D. S. Gorfein (Ed.), Resolving Semantic Ambiguity. New York: Springer-Verlag.

Gorfein, D. S., Viviani, J. M., \& Leddo, J. (1982). Norms as a tool for the study of homography. Memory \& Cognition, 10, 503-509.

Grey, S., \& Mathews, A. (2000). Effects of training on interpretation of emotional ambiguity. The Quarterly Journal of Experimental Psychology. A, Human Experimental Psychology, 53(4), 1143-1162. doi:10.1080/713755937

Haenze, M., \& Hesse, F. W. (1993). Emotional influences on semantic priming. Cognition \& Emotion, 7, 195-205. 
Halberstadt, J. B., Niedenthal, P. M., \& Kushner, J. (1995). Resolution of lexical ambiguity by emotional state. Psychological Science, 6, 278-282.

Hallion, L. S., \& Ruscio, A. M. (2011). A meta-analysis of the effects of cognitive bias modification on anxiety and depression. Psychological Bulletin, 137, 940-958.

Hermans, D., Crombez, G., \& Eelen, P.(2000). Automatic attitude activationand efficiency: The fourth horseman of automaticity. Psychologica Belgica, 40, $3-22$.

Hertel, P. T., \& Mathews, A. (2011). Cognitive bias modification: Past perspectives, current findings, and future applications. Perspectives on Psychological Science, 6, 521-536.

Hess, D. J., Foss, D. J., \& Carroll, P. (1995). Effects of global and local context on lexical processing during language comprehension. Journal of Experimental Psychology: General, 124(1), 62-82.

Harvey, A., Watkins, E., Mansell, W., \& Shafran, R. (2004). Cognitive Behavioural Processes Across Psychological Disorders: A Transdiagnostic Approach to Research and Treatment. Oxford University Press: Oxford, UK.

Hill, T, Lewicki, P., Czyzewska, M., \& Boss, A. (1989). Self-perpetuating development of encoding biases in person perception. Journal of Personality and Social Psychology, 57, 373-387.

Hino, Y., Lupker, S. J., \& Pexman, P. M. (2002). Ambiguity and synonymy effects in lexical decision, naming, and semantic categorization tasks: Interactions between orthography, phonology, and semantics. Journal of Experimental Psychology. Learning, Memory, and Cognition, 28(4), 686-713. doi:10.1037/0278-7393.28.4.686

Hirsch, C., \& Mathews, A. (1997). Interpretative inferences when reading about emotional events. Behaviour Research and Therapy, 35(12), 1123-1132. doi:10.1016/S0005-7967(97)00069-7

Hirsch, C.R., Hayes, S., \& Mathews, A. (2009). Looking on the bright side: Accessing benign meanings reduces worry. Journal of Abnormal Psychology, 118, 44-54.

Hirsch, C. R., Mathews, A. \& Clark, D. M. (2007). Inducing an interpretation bias changes self imagery: A preliminary investigation. Behaviour Research and Therapy, 45, 2173-2181

Holmes, E. A, \& Mathews, A. (2005). Mental imagery and emotion: A special relationship? Emotion,5(4), 489-497. doi:10.1037/1528-3542.5.4.489 
Holmes, E.A., Lang, T.J., \& Shah, D.M. (2009). Developing interpretation bias modification as a "cognitive vaccine" for depressed mood-Imagining positive events makes you feel better than thinking about them verbally. Journal of Abnormal Psychology, 118, 76-88.

Holmes, E. A., Mathews, A., Dalgleish, T., \& Mackintosh, B. (2006). Positive Interpretation Training: Effects of Mental Imagery Versus Verbal Training on Positive Mood. Behavior Therapy, 37(3), 237-247. doi:10.1016/j.beth.2006.02.002

Hoppitt, L., Mathews, A., Yiend, J., \& Mackintosh, B. (2010a). Cognitive bias modification: The critical role of active training in modifying emotional responses. Behavior Therapy, 41(1), 73-81. doi:10.1016/j.beth.2009.01.002

Hoppitt, L., Mathews, A., Yiend, J., \& Mackintosh, B. (2010b). Cognitive mechanisms underlying the emotional effects of bias modification, Applied Cognitive Psychology, 24(3), 312-325, http://doi.org/10.1002/acp

Kambe, G., Rayner, K., \& Duffy, S. A. (2001). Global context effects on processing lexically ambiguous words: evidence from eye fixations. Memory \& Cognition, 29(2), 363-372. doi:10.3758/BF03194931

Kawamoto, A. (1993). Nonlinear dynamics in the resolution of lexical ambiguity: A parallel distributed processing account. Journal of Memory and Language. doi: $10.1006 /$ jmla.1993.1026

Keuleers, E., \& Brysbaert, M. (2010). Wuggy: A multilingual pseudoword generator. Behavior Research Methods, 42(3), 627-633. doi:10.3758/BRM.42.3.627

Kissler, J., Assadollahi, R., \& Herbert, C. (2006). Emotional and semantic networks in visual word processing: insights from ERP studies. Progress in Brain Research, 156(6), 147-183. doi:10.1016/S0079-6123(06)56008-X

Kissler, J., \& Herbert, C. (2013). Emotion, Etmnooi, or Emitoon? - Faster lexical access to emotional than to neutral words during reading. Biological Psychology, 92(3), 464-479. doi:10.1016/j.biopsycho.2012.09.004

Klauer, K. C. (1997). Affective Priming. European Review of Social Psychology, 8(1), 67-103. doi:10.1080/14792779643000083

Koster, E. H. W., Fox, E., \& MacLeod, C. (2009). Introduction to the special section on cognitive bias modification in emotional disorders. Journal of Abnormal Psychology, 118(1), 1-4. doi:10.1037/a0014379

Kucera, H., \& Francis, W. N. (1967). Computational analysis of present-day American English. Providence, R1: Brown University Press 
Lang, P. J. (1994). The motivational organization of emotion: Affect-reflex connections. In S. VanGoozen, N. E. Van de Poll, J. A. Sergaeant (Eds.), Emotions: Essays on emotion theory.(pp. 61-93). Hillsdale, NJ: Erlbaum.

Lawson, C., MacLeod, C., \& Hammond, G. (2002). Interpretation revealed in the blink of an eye: Depressive bias in the resolution of ambiguity. Journal of Abnormal Psychology, 111(2), 321-328. doi:10.1037/0021-843X.111.2.321

Lewicki, P., Hill, T., \& Bizot, E. (1988). Acquisition of procedural knowledge about a pattern of stimuli that cannot be articulated. Cognitive Psychology, 20(1), 24-37. doi:10.1016/0010-0285(88)90023-0

Mackintosh, B., Mathews, A., Yiend, J., Ridgeway, V. \& Cook, E. (2006). Induced biases in emotional interpretation influence stress vulnerability and endure despite changes in context. Behavior Therapy, 37, 209-222. doi: 10.1016/j.beth.2006.03.001

MacLeod, C. M., Dodd, M. D., Sheard, E. D., Wilson, D. E., \& Bibi, U. (2003). In opposition to inhibition. Psychology of Learning and Motivation - Advances in Research and Theory, 43(416), 163-214. doi:10.1016/S00797421(03)01014-4

MacLeod, C., \& Mathews, A. (1991). Biased cognitive operations in anxiety: Accessibility of information or assignment of processing priorities? Behaviour Research and Therapy, 29(6), 599-610. doi:10.1016/00057967(91)90130-U

Marslen-Wilson, W. D. (1987). Functional parallelism in spoken wordrecognition. Cognition, 25(1-2), 71-102. doi:10.1016/0010-0277(87)900059

Masson, M. E., \& Borowsky, R. (1998). More than meets the eye: context effects in word identification. Memory \& Cognition, 26(6), 1245-1269.

Mathews, A. (2012). Effects of modifying the interpretation of emotional ambiguity. Journal of Cognitive Psychology, 24, 92-105.

Mathews, A., \& Mackintosh, B. (2000). Induced emotional interpretation bias and anxiety. Journal of Abnormal Psychology, 109, 602-615.

Mathews, A., Ridgeway, V., Cook, E., \& Yiend, J. (2007). Inducing a benign interpretational bias reduces trait anxiety. Journal of Behavior Therapy and Experimental Psychiatry, 38(2), 225-236. doi:10.1016/j.jbtep.2006.10.011

McNamara, D. S., \& McDaniel, M. A. (2004). Suppressing irrelevant information: Knowledge activation or inhibition? Journal of Experimental Psychology. Learning, Memory, and Cognition, 30(2), 465-482. doi:10.1037/02787393.30.2.465 
Medler, D.A., \& Binder, J.R. (2005). MCWord: An on-line orthographic database of the english language. http://www.neuro.mcw.edu/mcword/

Menne-Lothmann, C., Viechtbauer, W., Höhn, P., Kasanova, Z., Haller, S. P., Drukker, M., ... Lau, J. Y. F. (2014). How to boost positive interpretations? A meta-analysis of the effectiveness of cognitive bias modification for interpretation. PLOS ONE, 9(6). doi:10.1371/journal.pone.0100925

Meyer, D. E., \& Schvaneveldt, R. W. (1971). Facilitation in recognizing pairs of words: evidence of a dependence between retrieval operations. Journal of Experimental Psychology, 90(2), 227-234. doi:10.1037/h0031564

Mobini, S., Mackintosh, B., Illingworth, J., Gega, L., Langdon, P., \& Hoppitt, L. (2014). Effects of standard and explicit cognitive bias modification and computer-administered cognitive-behaviour therapy on cognitive biases and social anxiety. Journal of Behavior Therapy and Experimental Psychiatry, 45(2), 272-279. doi:10.1016/j.jbtep.2013.12.002

Mogg, K., Bradbury, K. E., \& Bradley, B. P. (2006). Interpretation of ambiguous information in clinical depression. Behaviour Research and Therapy, 44(10), 1411-1419. doi:10.1016/j.brat.2005.10.008

Morton, J. (1969). Interaction of information in word recognition. Psychological Review, 76(2), 165-178. doi:10.1037/h0027366

Nakic, M., Smith, B. W., Busis, S., Vythilingam, M., \& Blair, R. J. R. (2006). The impact of affect and frequency on lexical decision: The role of the amygdala and inferior frontal cortex. NeuroImage, 31(4), 1752-1761. doi:10.1016/j.neuroimage.2006.02.022

Neely, J. H. (1976). Semantic priming and retrieval from lexical memory: Evidence for facilitatory and inhibitory processes. Memory \& Cognition, 4(5), 648-654. doi:10.3758/BF03213230

Neely, J. H. (1977). Semantic priming and retrieval from lexical memory: Roles of inhibitionless spreading activation and limited-capacity attention. Journal of Experimental Psychology: General, 106(3), 226-254. doi:10.1037/00963445.106.3.226

Neely, J. H., Keefe, D. E., \& Ross, K. L. (1989). Semantic priming in the lexical decision task: roles of prospective prime-generated expectancies and retrospective semantic matching. Journal of Experimental Psychology. Learning, Memory, and Cognition, 15(6), 1003-1019. doi:10.1037/02787393.15.6.1003

Nelson, D. L., McEvoy, C. L., Walling, J. R., \& Wheeler, J. W. (1980). The university of south florida homograph norms. Behavior Research Methods \& Instrumentation, 12, 16-37. 
Nelis S., Vanbrabant K., Holmes E. A., \& Raes F. (2012). Greater positive affect change after mental imagery than verbal thinking in a student sample.Journal of Experimental Psychopathology, 3, 178 188. doi: $10.5127 /$ jep. 014811

New, B., Ferrand, L., Pallier, C., \& Brysbaert, M. (2006). Reexamining the word length effect in visual word recognition: new evidence from the English Lexicon Project. Psychonomic Bulletin \& Review, 13(1), 45-52. doi:10.3758/BF03193811

Nygaard, L. C., \& Lunders, E. R. (2002). Resolution of lexical ambiguity by emotional tone of voice. Memory \& Cognition, 30(4), 583-593. doi:10.3758/BF03194959

Oden, G. C., \& Spira, J. L. (1983). Influence of context on the activation and selection of ambiguous word senses. The Quarterly Journal of Experimental Psychology Section A, 35(1), 51-64. doi:10.1080/14640748308402116

Onifer, W., \& Swinney, D. A. (1981). Accessing lexical ambiguities during sentence comprehension: Effects of frequency of meaning and contextual bias. Memory \& Cognition, 9(3), 225-236. doi: 10.3758/BF03196957

Palazova, M. (2014). Where are emotions in words? Functional localization of valence effects in visual word recognition. Frontiers in Psychology, 5, 20132015. doi:10.3389/fpsyg.2014.01105

Posner, M. I., \& Snyder, C. R. (1975). Attention and cognitive control. In R. L. Solso (Ed.), Information processing and cognition (pp. 55-85). Hillsdale, NJ: Erlbaum.

Rapee, R. M., \& Heimberg, R. G. (1997). A cognitive-behavioral model of anxiety in social phobia. Behaviour Research and Therapy, 35, 741-756. doi:10.1016/S0005-7967(97)00022-3

Richards, A, \& French, C. C. (1992). An anxiety-related bias in semantic activation when processing threat/neutral homographs. The Quarterly Journal of Experimental Psychology. A, Human Experimental Psychology, 45(3), 503525. doi:10.1080/02724989208250625

Ruiz-Caballero, J. a. R., \& Moreno, J. B. (1992). Individual difference in depression, induced mood, and perception of emotionally toned words. European Journal of Personality, 6(3), 215-224. doi:10.1002/per.2410060304

Salemink, E., Hertel, P., \& Mackintosh, B. (2010). Interpretation training influences memory for prior interpretations. Emotion, 10(6), 903-907. doi:10.1037/a0020232 
Salemink, E., van den Hout, M., \& Kindt, M. (2009). Effects of positive interpretive bias modification in highly anxious individuals. Journal of Anxiety Disorders, 23(5), 676-683. doi:10.1016/j.janxdis.2009.02.006

Salemink, E., van den Hout, M., \& Kindt, M. (2010). How does Cognitive Bias Modification affect anxiety? Mediation analyses and experimental data. Behavioural and Cognitive Psychotherapy, 38(1), 59-66. doi:10.1017/S1352465809990543

Schacht, A., \& Sommer, W. (2009). Time course and task dependence of emotion effects in word processing. Cognitive, Affective \& Behavioral Neuroscience, 9(1), 28-43. doi:10.3758/CABN.9.1.28

Schneider, W., Eschman, A., \& Zuccolotto, A. (2002) E-Prime User's Guide. Pittsburgh:Psychology Software Tools Inc.

Schvaneveldt, R. W., \& Meyer, D. E. (1976). Lexical ambiguity, semantic context, and visual word recognition. Journal of Experimental Psychology. Human Perception and Performance, 2(2), 243-256.

Schvaneveldt, R.W., \& Meyer, D.E. (1973). Retrieval and comparison processes in semantic memory, in Kornblum, S. (Ed.). Attention and performance IV (pp. 395-409). New York: Academic Press.

Schvaneveldt, R. W., Meyer, D. E., \& Becker, C. W. (1976). Lexical ambiguity, semantic context, and visual word recognition. Journal of Experimental Psychology: Human Perception and Performance, 2,243-256.

Schwarz, N., \& Clore, G. L. (1983). Mood, misattribution, and judgments of wellbeing: Informative and directive functions of affective states. Journal of Personality and Social Psychology, 45(3), 513-523. doi:10.1037/00223514.45.3.513

Segal, Z. V., \& Ingram, R. E. (1994). Mood priming and construct activation in tests of cognitive vulnerability to unipolar depression. Clinical Psychology Review, 14(7), 663-695. doi:10.1016/0272-7358(94)90003-5

Seidenberg, M. S., \& McClelland, J. L. (1989). A distributed, developmental model of word recognition and naming. Psychological Review, 96, 523-568.

Seidenberg, M.S. (1985). The time course of information activation and utilization in visual word recognition. In D. Besner, T.G. Waller, \& E.M. MacKinnon (Eds.), Reading research: Advances in theory and practice (pp. 199-252). New York: Academic Press.

Seidenberg, M. S., \& McClelland, J. L. (1989). A distributed, developmental model of word recognition and naming. Psychological Review, 96(4), 523-568. doi:10.1037/0033-295X.96.4.523 
Shifrin, R. M., \& Schneider, W. (1977). Controlled and automatic human information processing: II. Perceptual learning, automatic attending, and a general theory. Psychological Review, 84, 127-190.

Simpson, G. B. (1984). Lexical ambiguity and its role in models of word recognition. Psychological Bulletin, 96(2), 316-340. doi:10.1037/00332909.96.2.316

Simpson, G. B. (1994). Context and the Processing of Ambiguous Words. In M. A. Gernsbacher (Ed.), Handbook of psycholinguistic research (pp. 359-374). San Diego, CA: Academic Press.

Simpson, G. B., \& Burgess, C. (1985). Activation and selection processes in the recognition of ambiguous words. Journal of Experimental Psychology: Human Perception and Performance, 11(1), 28-39. doi:10.1037/00961523.11.1.28

Simpson, G. B., \& Kang, H. (1994). Inhibitory processes in the recognition of homograph meanings. In D. Dagenbach \& T. H. Carr (Eds.), Inhibitory processes in attention memory and language (pp. 359-382). San Diego, CA: Academic Press.

Spruyt, A., Hermans, D., De Houwer, J., Vandromme, H., \& Eelen, P. (2007). On the nature of the affective priming effect: effects of stimulus onset asynchrony and congruency proportion in naming and evaluative categorization. Memory \& Cognition, 35(1), 95-106. doi:10.3758/BF03195946

Standage, H., Ashwin, C., \& Fox, E. (2009). Comparing visual and auditory presentation for the modification of interpretation bias. Journal of Behavior Therapy and Experimental Psychiatry, 40(4), 558-570. doi:10.1016/j.jbtep.2009.07.006

Steinman, S. A. \& Teachman, B. A. (2010). Modifying interpretations among individuals high in anxiety sensitivity. Journal of Anxiety Disorders, 24, 7178. doi:10.1016/j.janxdis.2009.08.008

Stopa, L., \& Clark, D. M. (2000). Social phobia and interpretation of social events. Behaviour Research and Therapy, 38(3), 273-283. doi:10.1016/S00057967(99)00043-1

Swinney, D. A. (1979). Lexical access during sentence comprehension: (Re) Consideration of context effects. Journal of Verbal Leaning and Learning Behavior, 18, 645-659

Tanenhaus, M. K., Leiman, J. M., \& Seidenberg, M. S. (1979). Evidence for multiple stages in the processing of ambiguous words in syntactic contexts. Journal of Verbal Learning and Verbal Behavior, 18(4), 427-440. doi:10.1016/S00225371(79)90237-8 
Tran, T. B., Hertel, P. T., \& Joormann, J. (2011). Cognitive bias modification: Induced interpretive biases affect memory. Emotion, 11(1), 145-52. http://doi.org/10.1037/a0021754

Twilley, L. C., \& Dixon, P. (2000). Meaning resolution processes for words: A parallel independent model. Psychonomic Bulletin \& Review, 7(1), 49-82.

Twilley, L. C., Dixon, P., Taylor, D., \& Clark, K. (1994). University of Alberta norms of relative meaning frequency for 566 homographs. Memory \& Cognition, 22, 111-126.

Tyler, L. K. (1984). The structure of the initial cohort: evidence from gating. Perception \& Psychophysics, 36(5), 417-427. doi:10.3758/BF03207496

Vassilopoulos, S. P., Banerjee, R., \& Prantzalou, C. (2009). Experimental modification of interpretation bias in socially anxious children: Changes in interpretation, anticipated interpersonal anxiety, and social anxiety symptoms. Behaviour Research and Therapy, 47(12), 1085-1089. doi:10.1016/j.brat.2009.07.018

Warriner, A. B., Kuperman, V., \& Brysbaert, M. (2013). Norms of valence, arousal, and dominance for 13,915 English lemmas. Behavior Research Methods, 45, 1191-1207.

West, R. F., \& Stanovich, K. E. (1982). Source of inhibition in experiments on the effect of sentence context on word recognition. Journal of Experimental Psychology: Learning, Memory, and Cognition, 8(5), 385-399. doi:10.1037/0278-7393.8.5.385

Wiley, J., \& Rayner, K. (2000). Effects of titles on the processing of text and lexically ambiguous words: evidence from eye movements. Memory \& Cognition, 28(6), 1011-1021. doi:10.3758/BF03209349

Wilson, M.D. (1988) The MRC Psycholinguistic Database: Machine Readable Dictionary, Version 2. Behavioural Research Methods, Instruments and Computers, 20(1), 6-11.

Wilson, E. J., MacLeod, C., Mathews, A. \& Rutherford E. M. (2006). The causal role of interpretive bias in anxiety reactivity. Journal of Abnormal Psychology, 115, 103-111. doi: 10.1037/0021-843X.115.1.103.

Woud, M. L., \& Becker, E. S. (2014). Editorial for the special issue on cognitive bias modification techniques: An introduction to a time traveller's tale. Cognitive Therapy and Research, 38(2), 83-88. doi:10.1007/s10608-0149605-0

Yiend, J. (2004) (Ed.). Cognition, emotion and psychopathology. Cambridge, U.K.: Cambridge University Press. 